Florida International University FIU Digital Commons

7-9-2008

\title{
The Relationship of Fear of Intimacy, Body Image Avoidance and Body Mass Index
}

Elliot Montgomery Sklar

Florida International University, elliotmontgomery@gmail.com

DOI: $10.25148 /$ etd.FI08121918

Follow this and additional works at: https://digitalcommons.fiu.edu/etd

\section{Recommended Citation}

Sklar, Elliot Montgomery, "The Relationship of Fear of Intimacy, Body Image Avoidance and Body Mass Index" (2008). FIU Electronic Theses and Dissertations. 15.

https://digitalcommons.fiu.edu/etd/15

This work is brought to you for free and open access by the University Graduate School at FIU Digital Commons. It has been accepted for inclusion in FIU Electronic Theses and Dissertations by an authorized administrator of FIU Digital Commons. For more information, please contact dcc@fiu.edu. 


\section{FLORIDA INTERNATIONAL UNIVERSITY}

Miami, Florida

\section{THE RELATIONSHIP OF FEAR OF INTIMACY, BODY IMAGE AVOIDANCE AND BODY MASS INDEX}

A dissertation submitted in partial fulfillment of the

requirements for the degree of

DOCTOR OF PHILOSOPHY

in

PUBLIC HEALTH

by

Elliot Montgomery Sklar

2008 
To: Dean Fernando M. Trevino

Dr. Robert R. Stempel School of Public Health

This dissertation, written by Elliot Montgomery Sklar, and entitled The Relationship of Fear of Intimacy, Body Image Avoidance and Body Mass Index, having been approved in respect to style and intellectual content, is referred to you for judgment.

We have read this dissertation and recommend that it be approved.

Jessy Devieux

Luther Brewster

Yolanda Cal

Robert Malow, Major Professor

Date of the Defense: July 9, 2008

The Dissertation of Elliot Montgomery Sklar is approved.

Dean Fernando M. Trevino

Dr. Robert R. Stempel School of Public Health

Dean George Walker

University Graduate School

Florida International University, 2008 
(C) Copyright 2008 by Elliot Montgomery Sklar

All rights reserved. 


\section{DEDICATION}

This dissertation is dedicated to those for whom this research may provide insight and a new perspective on body weight; ways to conceive of its meaning and its loss. There is no doubt that without the support of my partner and family, this journey toward my doctoral dissertation would not have been possible. Matthew, you have stood by my side on the days of triumph and on those when the destination on this journey seemed out of sight. I will forever be grateful for your unwavering love and support. My family Hannah, Stanley \& Shawn - thank you for providing a foundation and consistency that has fostered the will, perseverance and determination to achieve whatever I have sought out in my life. Your unconditional regard for my intellectual and personal development has been fundamental in my life and has led to this noble beginning. 


\section{ACKNOWLEDGMENTS}

I would like to thank my major professor, Dr. Robert Malow for his mentoring and guidance, belief in my potential and this research, and whose knowledge, expertise and sense of humor were integral to my success at Florida International University. I would also like to thank Dr. Jessy Devieux for her direction and encouragement, Dr. Luther Brewster for his constant willingness to show support and provide direction. I would like to extend a special recognition to Dr. Yolanda Cal, whose strength of character, great will, and vested interest in this project and its findings were undoubtedly a force that inspired my own strength at difficult times on this journey. Dr. Alan Carsrud cannot go unmentioned; his wisdom and counsel helped me to navigate to this point. I would also like to recognize Dr. H. Virginia McCoy \& Dr. Michele Ciccazzo for their committed support and dedication to helping me realize this wonderful accomplishment for myself and for the Department of Health Promotion \& Disease Prevention. 


\section{ABSTRACT OF THE DISSERTATION \\ THE RELATIONSHIP OF FEAR OF INTIMACY, BODY IMAGE AVOIDANCE AND BODY MASS INDEX}

by

Elliot Montgomery Sklar

Florida International University, 2008

Miami, Florida

\section{Professor Robert M. Malow, Major Professor}

Issues of body image and ability to achieve intimacy are connected to body weight, yet remain largely unexplored and have not been evaluated by gender. The underlying purpose of this research was to determine if avoidant attitudes and perceptions of one's body may hold implications toward its use in intimate interactions, and if an above average body weight would tend to increase this avoidance. The National Health and Nutrition Examination Survey (NHANES, 1999-2002) finds that $64.5 \%$ of US adults are overweight, with $61.9 \%$ of women and $67.2 \%$ of men. The increasing prevalence of overweight and obesity in men and women shows no reverse trend, nor have prevention and treatment proven effective in the long term.

The researcher gathered self-reported age, gender, height and weight data from 55 male and 58 female subjects (determined by a prospective power analysis with a desired medium effect size $(r=.30)$ to determine body mass index (BMI), determining a mean age of 21.6 years and mean BMI of 25.6. Survey instruments consisted of two scales that are germane to the variables being examined. They were (1) Descutner and Thelen of the 
University of Missouri's (1991) Fear-of-Intimacy scale and (2) Rosen, Srebnik, Saltzberg, and Wendt's (1991) Body Image Avoidance Questionnaire.

Results indicated that as body mass index increases, fear of intimacy increases $(p<0.05)$ and that as body mass index increases, body image avoidance increases $(p<0.05)$. The relationship that as body image avoidance increases, fear of intimacy increases was not supported, but approached significance at $(p<0.07)$. No differences in these relationships were determined between gender groups. For age, the only observed relationship was that of a difference between scores for age groups [18 to 22 (group 1) and ages 23 to 34 (group 2)] for the relationship of body image avoidance and fear of intimacy $(p<0.02)$. The results suggest that the relationship of body image avoidance and fear of intimacy, as well as age, bear consideration toward the escalating prevalence of overweight and obesity. An integrative approach to body weight that addresses issues of body image and intimacy may prove effective in prevention and treatment. 


\section{TABLE OF CONTENTS}

CHAPTER

PAGE

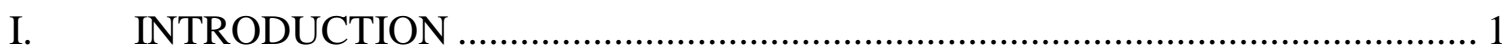

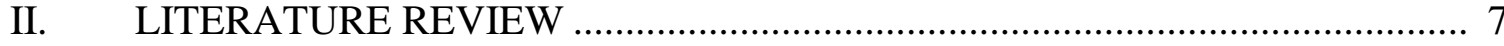

Obesity’s Impact Upon Body Image ................................................................. 7

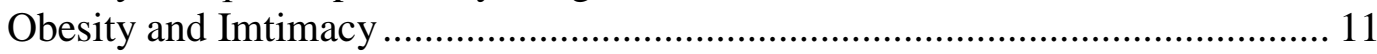

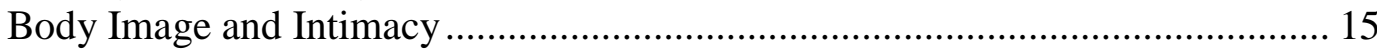

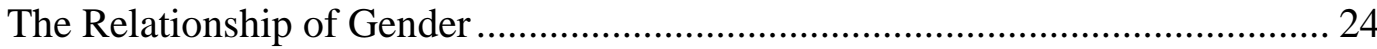

Body Images: Respresentations of Race, Culture and Ethnicity ........................ 31

The Relationship of Fear of Intimacy, Body Image Avoidnace and Body Mass

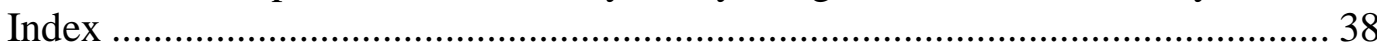

Research Questions and Hypotheses ......................................................... 44

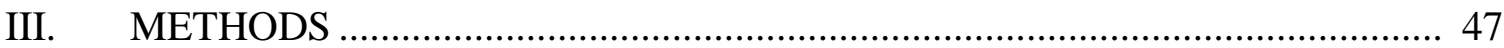

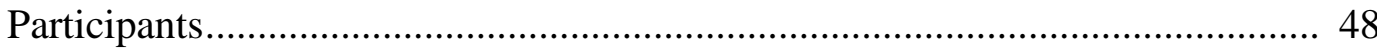

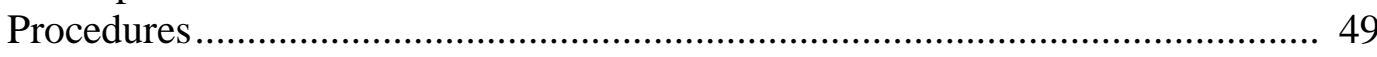

Measures ................................................................................................ 50

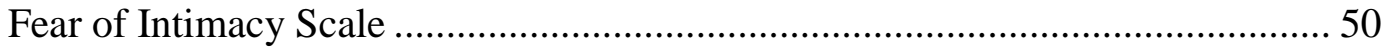

Body Image Avoidance Questionnaire ......................................................... 51

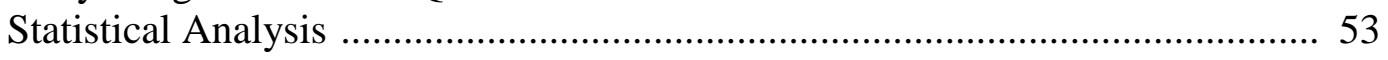

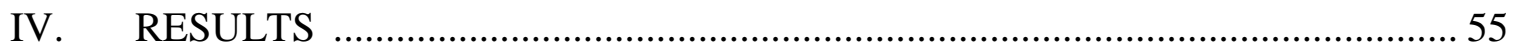

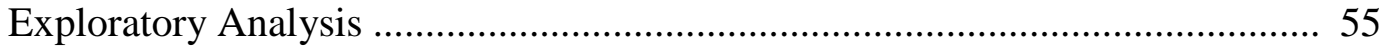

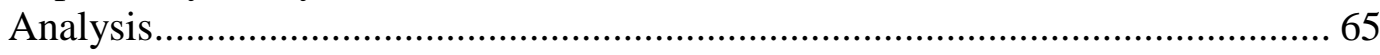

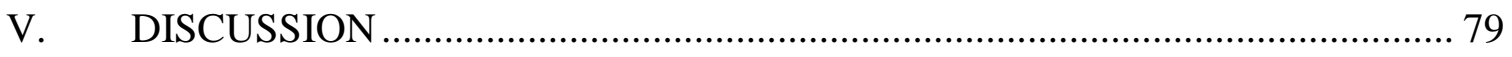

The Relationship of Body Mass Index and Fear of Intimacy ............................ 79

The Relationship of Body Image Avoidance and Fear of Intimacy .................. 82

The Relationship of Gender and Fear of Intimacy, Body Mass Index and Body Image Avoidance .............................................................................. 84

The Relationship of Body Image Avoidance and Fear of Intimacy Between Age

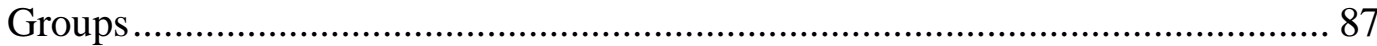

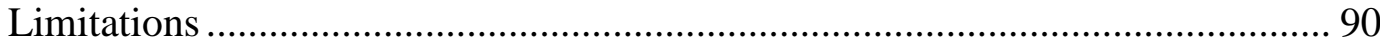

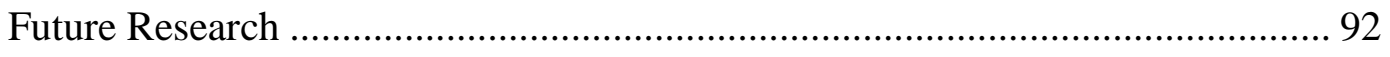

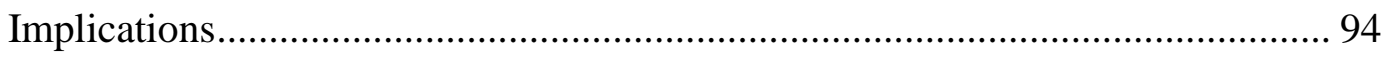

LIST OF REFERENCES................................................. 102

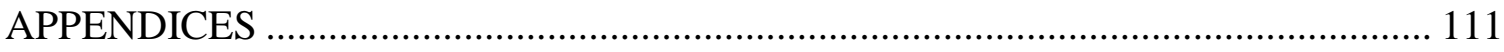

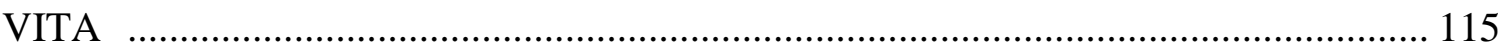




\section{LIST OF TABLES}

TABLES

PAGE

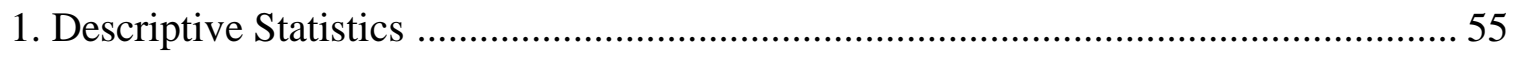

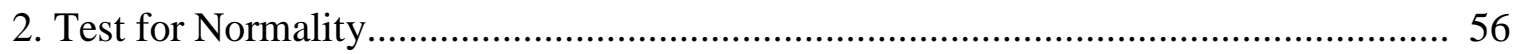

3. Matrix of Correlations Between Major Variables ..................................................... 65

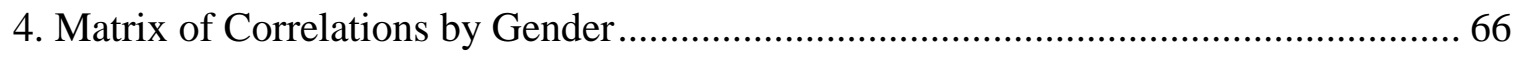

5. Test of Equality of Population Correlations by Gender................................................. 67

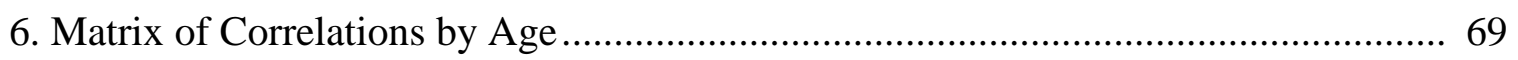

7. Test of Equality of Population Correlations by Age ................................................ 69 


\section{LIST OF GRAPHS}

GRAPHS

PAGE

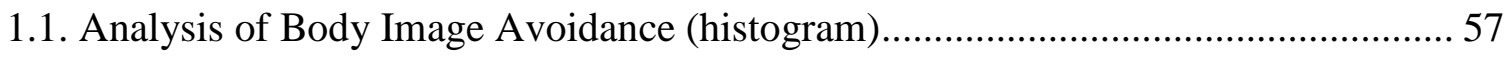

1.2. Analysis for Body Image Avoidance (quantile plot) ...................................... 58

1.3. Analysis for Body Image Avoidance (natural logarithm) ................................ 59

1.4. Analysis for Body Image Avoidance (square root) ........................................... 59

1.5. Analysis for Body Image Avoidance (log 10 base) ............................................ 59

2.1. Analysis of Fear of Intimacy (histogram) .................................................... 60

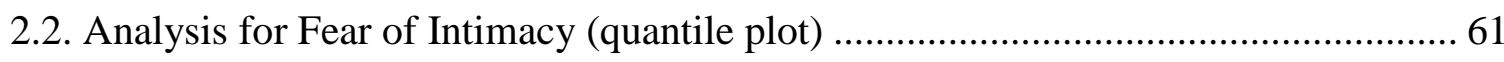

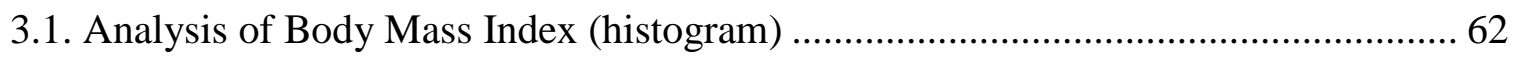

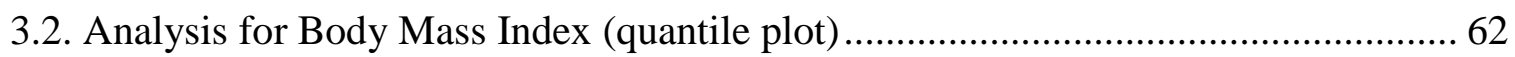

4.1. Scatterplot: Body Mass Index \& Body Image Avoidance ................................... 63

4.2. Scatterplot: Fear of Intimacy \& Body Image Avoidance .................................. 64

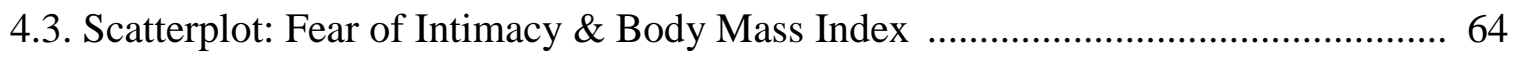

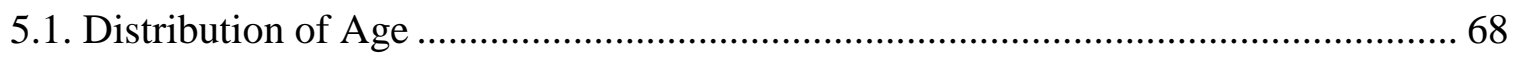

6.1. Scatterplot: Fear of Intimacy \& Body Mass Index by Gender ........................... 71

6.2. Scatterplot: Fear of Intimacy \& Body Image Avoidance by Gender..................... 72

6.3. Scatterplot: Body Image Avoidance \& Body Mass Index .................................. 74

7.1. Scatterplot: Fear of Intimacy \& Body Mass Index by Age .............................. 75

7.2. Scatterplot: Fear of Intimacy \& Body Image Avoidance by Age.......................... 76

7.3. Scatterplot: Body Mass Index \& Body Image Avoidance by Age ...................... 77 


\section{ACRONYMS AND ABBREVIATIONS}

AOA

AIDS

BIA

BDS

BMI

BRFSS

FOI

FOIS

CDC

DSM

EDI

FDOH

HBM

IOM

MSIS

NCHS

NHANES

NIH

SMART

WHO
American Obesity Association

Acquired Immune Deficiency Syndrome

Body Image Avoidance

Body Dissatisfaction Scale

Body Mass Index

Behavioral Risk Factor Surveillance System

Fear of Intimacy

Fear of Intimacy Scale

Center for Disease Control

Diagnostic and Statistical Manual of Mental

Disorders

Eating Disorder Inventory

Florida Department of Health

Health Belief Model

Institute of Medicine

Miller Social Intimacy Scale

National Center for Health Statistics

National Health and Nutrition Examination Survey

National Institutes of Health

Selected Metropolitan / Micropolitan Area Risk

World Health Organization 


\section{CHAPTER I}

\section{INTRODUCTION}

The National Health and Nutrition Survey (NHANES, 1999-2002) finds that at present 64.5 percent of US adults are overweight, with 61.9 percent of women and 67.2 percent of men. Of this, 30.5 percent of adults are obese, comprised of 33.4 percent women and 27.5 percent men ("Statistics," 2006). Despite a higher overweight prevalence amongst men, weight loss and weight preoccupation is still socially considered a gendered (female) preoccupation (Brumberg, 1997; Jagsdaidt et al, 1997; Areton, 2005).

According to the World Health Organization (1997), obesity is defined as a body mass index (BMI) above $30 \mathrm{~kg} / \mathrm{m}^{2}$, while overweight is defined as a BMI above $25 . \mathrm{kg}^{2}$. Morbid obesity occurs at a BMI above $40 \mathrm{~kg} / \mathrm{m}^{2}$. Overweight and obesity are found worldwide, and the prevalence of these conditions in the US rank high along with other developed nations (“Statistics," 2006). The National Center for Health Statistics (NCHS) and the National Health and Nutrition Survey (NHANES) have compiled age-adjusted data in conjunction with the US Census Bureau "Census 2000" results (Flegal, 2005). The Selected Metropolitan / Micropolitan Area Risk Trends / Behavioral Risk Factor Surveillance System (SMART BRFSS, 2002) information acquired for Miami - MiamiBeach - Kendall, FL Metropolitan Division ("Statistics," 2006) indicates an obesity rate across the board consistent with national averages (20.5\% versus a national $21.9 \%)$, while exercise levels are substantially higher (36.7\% versus a national 24.1\%). Although a high degree of heritability for obesity exists, the aforementioned rapid rise in obesity 
prevalence suggests that environmental factors may be responsible for obesity within some cultures and countries (Smuts, 1992; Nezlek, 1999; "Statistics," 2006).

The consumption of food, at its most base level, is to satisfy physiological hunger (Maslow, 1943). Psychologists concerned with theories of motivation would note that an excessive caloric intake resulting in overweight and obesity may also serve to satisfy other needs; this consideration sparked the psychoanalytic approach to understanding obesity in the 1950s (Beller, 1977). The 1952 Rorschach study of 81 obese female respondents by Kotlov \& Muanski indicated a deep repressive tendency in their study sample, comparable to neurotics (Beller, 1977). The paradoxical question remained - if this sample of women were found to be more repressive, why then, would they not repress the urge to overeat? Approached at first through Freudian analogies, symbolism and exploratory criteria, it is no surprise that attitudes related directly to the psychoanalytic discourse on sex. Confusion about sex, size and body image identified in obese study samples later reemerged as themes of gender and sexual confusion. Researchers had noted that obese samples seemed to experience greater challenges toward sex roles than their normal-weighted counterparts (Beller, 1977; Bess, 1997). The point is not an unimportant one; sex is a fundamental, physiological need not unlike hunger. Furthermore, ways of dealing with bodily needs run the gamut from voracity to total repression (Maslow, 1943; Bess, 1997).

When considering the framework of motivational psychologists such as Maslow, the disconnect becomes apparent; our society almost overwhelmingly demands a slender figure - yet we continue to grow (Smuts, 1992; Nezlek, 1999; "Statistics," 2006). Conceptions of obesity as socially undesirable have been widely marketed over the past 
century, particularly where sexuality is concerned (Beller, 1977). Learned social behavior can modify sexual activity based upon the positive and negative attributes ascribed to body image that impact upon self-esteem (Strauss \& Wise, 1978), and weight bears significant social implications. Researchers have found that the attitude of the obese person toward body and sexuality was a determining factor in the prediction of sexual functioning, establishing a directionality of the relationship between intimacy and body image (Areton, 2002; Adolfsson, et al. 2004).

Feminist theorists support the concept that traditionally male-driven industries including media, advertising, cosmetics, etc. have conspired to promote widespread dissatisfaction of women's bodies (Brumberg, 1988). It is common and expected that women be unhappy with their bodies; this feeling has been termed "normative discontent" (Tiggemann \& Lynch, 2001). This normative discontent, when applied to the aforementioned early psychoanalytic theories on obesity, may include sexuality (as experienced through intimacy) as a modern extension of this normative discontent in a society consumed with sexual liberties (Brumberg, 1988; Brumberg; 1997).

Those who perceive their bodies in negative terms are obviously more likely to be sexually avoidant (Cash, 1993). While psychoanalytic approaches have fallen out of vogue when addressing obesity, modern individual and community approaches to weight management have proven largely ineffective (Beach \& Martin, 1985; Choi et al., 2005; "Statistics", 2006). Neither physicians nor patients are accurately aware of the topics that underlay obesity and excessive consumption of food, leaving the true psychological issues untreated and may explain poor results where long term weight control are concerned (Beach \& Martin, 1985). Where obesity is concerned, it has been suggested 
that this self-imposed physique serves as a distraction from other psychopathology (Jagsdaidt et al., 2001). As overweight and obesity have become the norm, ("Statistics", 2006) this described psychopathology concerning body image and intimacy may no longer be considered abnormal, but rather another normative discontent worthy of consideration toward understanding this epidemic.

Health behavior efforts have examined the interaction of individuals and environments as follows:

1) Behavioral settings were viewed as having a direct effect on behavior.

2) Behavioral settings leave under the direct control of microenvironmental agents.

3) Macroenvironmental agents as having indirect yet powerful control over policy and regulation.

For obesity, individually-based approaches have been viewed as largely ineffective because they do nothing to alter the environmental factors that contribute to obesity (Beach \& Martin, 1985; Friedman \& Brownell, 1995; Lang \& Froelicher, 2006). According to the 2006 study by Lang \& Froelicher, factors involved in the development of overweight and obesity include: individual behavior, environmental cues, policy and regulation from micro to macro levels of government.

Health behavior interventions have advocated a two-step approach for comprising assessment and management when considering obese adults for evaluation and treatment (Lang \& Froelicher, 2006):

1) Medical and weight assessment and classification.

2) Behavioral assessment - to obtain biological and behavioral factors contributing to the individual's obesity, assess psychosocial consequences of excessive weight, 
examine individual's goals and expectations for weight reduction and consider the degree of motivation to attain these goals.

The epidemic of overweight and obesity in men and women continues to escalate and results in increased risk for diseases and diminished quality of life. Considerable research exists on obesity and on sexuality as separate issues; few theories address their interrelation. Psychoanalytic theory provides a framework for better understanding these issues within the individual, while body image and intimacy can be examined from the perspective of social psychology (Friedman \& Brownell, 1995; Brumberg, 1997; Jagsdaidt et al., 2001; Cash \& Pruzinsky, 2002). An integrative framework that considers the individual in a social context can, perhaps, lend to better preventative and treatment approaches. The lack of research by sexologists or by obesity experts on the interrelation of the two issues resonate with cultural mores and fail to find inclusion in weight management practices. As a result, the notion that expressing sexuality is relegated to the thin may result in the interpretation of intimacy as threatening for those who are overweight or obese.

Body dissatisfaction among children and adolescents has been shown to result in avoidant behavior (Brumberg, 1988; Grogan, 1999; Haines \& Neumark-Sztainer, 2006). Avoidant behaviors, including isolation, often involve lower levels of physical activity and may bear implications later in life when forming intimate relationships (Haines \& Neumark-Sztainer, 2006). Body image dissatisfaction is well established as a risk factor for the development of eating disorders [according to the Diagnostic and Statistical Manual of Mental Disorders (DSM IV) criteria] but has rarely been considered toward 
the incidence of overweight and obesity. Public health efforts aimed at the prevention of overweight and obesity may find that community-based health promotion and interventions aimed at improving body satisfaction have implications for the prevention of obesity and eating disorders across the lifespan.

The primary purpose of this study sought to examine the relationship between a fear-of-intimacy and body image avoidance (attitudinal and perceptual), and how these relationships may be affected by body mass index. A secondary purpose sought to examine how these relationships may be gendered. These relationships have not been considered toward understanding what may be a cognitive reasoning for consumption that has contributed to the increase in rates of overweight and obesity since 1980 (Flegal, 2005; "Statistics," 2006). The dearth of scientific literature pertaining to the gendered relationship of fear of intimacy, body image avoidance and body mass index fails to provide insight into dimensions of this relationship that may be relevant to understanding the epidemic of obesity in relation to social changes in our culture. 


\section{CHAPTER II}

\section{LITERATURE REVIEW}

\section{Obesity’s Impact upon Body Image}

In 2005, the American Obesity Association (AOA) reported upon the significant impact of morbid obesity (BMI above $40 \mathrm{~kg} / \mathrm{m}^{2}$ ) on quality of life with regards to physical intimacy, social interactions and employment opportunities to comprise some of the barriers faced by this segment of the population (“American Obesity Association”, 2005). This was the first report of its kind to address sexuality of the obese in such a fashion. The study, which surveyed 519 obese Americans (aged 21 to 72), indicated that of the nearly 40 percent of surveyed participants in committed relationships, their frequency of sexual intimacy with their partners was less than once per month. By comparison, only 17 percent of the general population reported frequency of sexual intimacy at less than once per month. The survey also reported that during these intimate moments, weight resulted in feelings of embarrassment, furthering physical and emotional withholding and served to impact the frequency of intimacy - if able to be physically intimate at all ("American Obesity Association", 2005). It would seem reasonable to believe that a linear relationship may exist between body weight and intimacy, also affecting those persons who experience obesity to a lesser degree (BMI below $40 \mathrm{~kg} / \mathrm{m}^{2}$ ).

Another study presented by the American Obesity Association (2005) followed 928 obese men and women (as classified by BMI). Of those seeking treatment for their obesity, 50\% had a decreased sex drive and trouble wanting sex, $42 \%$ felt they had 
problems performing sexually, and $41 \%$ said they avoided sex altogether ("American Obesity Association”, 2005). This research, presented by the Duke University Medical Center to the American Obesity Association, showed that morbidly obese Americans were 25 times more likely than those of normal weight to report problems with their sex lives. This is an important and often overlooked issue as the obese are rarely depicted in a sexual light in mainstream media, nor often considered in sexual terms in research studies (Areton, 2002; “American Obesity Association”, 2005; Jagsdaidt et al., 2001).

Research has moved toward considering body-image dissatisfaction as a powerful mediator for the emotional quality of life of obese individuals (Friedman \& Brownell, 1995; Jagsdaidt et al., 2001; Cash \& Pruzinsky 2002). Friedman et al. (2002) proposed several additional risk factors that may determine which obese individuals will suffer negative psychological consequences. These factors included social class, degree of obesity, and body-image dissatisfaction. In this 2002 study, body-image dissatisfaction was identified as a potential mediator of the relationship between dysphoric psychological states (including depression and low self-esteem) and obesity in a treatment-seeking population. Although it was long assumed that body image was a unidimensional construct, recent research has suggested a multi-dimensional structure with regards to body image including perceptual body image (i.e., estimation of one's body size) and attitudinal body image (i.e., affective, cognitive, and behavioral concerns with one's body size). It would seem reasonable to propose that the pervasiveness of the thin body ideals that remain in Western fashion would have an even greater impact on individuals who are, in fact, overweight. 
A 2007 study examined appearance-based rejection sensitivity as an extension of body image dissatisfaction (Park, 2007). This appearance-based rejection sensitivity was characterized by anxious concerns and expectations about being rejected based on one's perceived physical attractiveness. The study found that subjects who engaged in selfaffirmation (thought of their personal strengths) or experienced a secure attachment through a close, caring relationship, were buffered from the negative effects of an appearance threat on subsequent self-esteem and mood. The study found that those preoccupied with their bodies would be motivated to engage in behaviors to appear attractive and avoid rejection by others and therefore were expected to be related to greater symptoms of disordered eating (Park, 2007). This finding, however, would suggest that as thin is the desirable condition in our society, individuals would be preoccupied with achieving a healthy body weight, and yet the prevalence of obesity continues to rise. The study did not examine body mass index, nor the prevalence of eating disorders in the study sample and failed to consider the importance of these relationships with regard to body image.

As the social pressures for thinness and the associated stigmatization of obesity have increased, so has the rate of obesity (Friedman \& Brownell, 1995; “American Obesity Association", 2005; "Statistics," 2006). Among the overweight and obese segments of the population, as the pressure for thinness increases, the discrepancy between desirable body weight and actual body weight increases as well. This relationship has served to intensify the body-image concerns of those who are in fact overweight, and speculated that the greater the dissatisfaction, the greater the resulting 
avoidance toward social interaction (Friedman \& Brownell, 1995; “American Obesity Association", 2005; Park, 2007). In spite of the growing body of research, little has been reported to suggest a baseline for, nor implications of the relationship between body image and body weight, nor how intimacy may be part of this equation. Contributions from this research over the past decade have failed to find inclusion in prevention and treatment approaches to obesity.

Fitzgibbon et al. (2000) suggests that differences in obesity may be related to differences in body image within the obese cohort of the population. While support had been established for a model of body dissatisfaction defined as a discrepancy between current body image and ideal body image, the study determined that a discrepancy between current and ideal body image is not necessarily comparable with perception of being overweight. This is an important consideration for the heterogeneous nature of western cultures, reflected in the studies and implications attached to the population at hand (Fitzgibbon et al, 2000; Lovejoy, 2001). Perceptions of being overweight may occur before or after there is a discrepancy between the current and ideal body image for men and women (Fitzgibbon et al., 2000).

A discrepancy between an individual's current and ideal body image may reflect body dissatisfaction and may be an important factor which encourages attention to body weight (often measured as BMI). The limitations of available research fail to examine a relationship of body mass index and body image avoidance - the implications of which are important in considering the increasing prevalence of overweight and obesity in our society. 


\section{Obesity and Intimacy}

Excessive weight (as defined by BMI) carries psychological consequences in developed societies that value thinness and physical attraction; the resulting affect may motivate avoidant cognitions and behaviors. Body-image factors (attitudinal and perceptual) conspire to discriminate obese from their non-obese counterparts and manifest in avoidant behavior (Cash, 1993). Obese individuals overestimate or distort the size of their body more, are more dissatisfied and preoccupied with their appearance, and tend to avoid more social interactions because of their appearance than normal weight individuals (Cash, 1990).

Wise (1978), Shapiro (1980), and Spiegel (1988) have established that obesity, in itself, does not affect sexual functioning. Wise (1978) studied twenty-three morbidly obese individuals (as defined by BMI), 13 men and 10 women, who were screened for ileal bypass surgery and evidence of psychiatric illness. Following the examination of a detailed sexual history to determine frequency of activity, libidinal drive, self-concept and body image, it was concluded that these morbidly obese subjects displayed a sense of shame and low esteem about their attractiveness. Shapiro (1980) administered a 137-item questionnaire about sexual attitudes and behaviors to a sample of one hundred Caucasian females, divided into two comparison groups on the basis of weight. The results indicated that the cohort of obese women had a general, pervasively negative self-concept, felt more inhibited in discussing sex with their partners, rated their primary relationships with discontent, and compared their physical attractiveness negatively to that of other women around them. Spiegel's (1988) study examined differences between obese women in 
relationships and those not in relationships. The women in relationships scored higher in self-satisfaction and self-esteem and revealed a mean average weight of eleven pounds less than their sampled counterpart. The obese women placed greater emphasis on their weight as a cause of not having a relationship, supporting the hypothesis that attitudes and not inherent psychological problems nor obesity, as such, may be responsible for influencing sexual functioning. The resulting question remains - does a fear of intimacy arise from issues of sexuality, or as an expression of body image avoidance due to body weight?

Researchers have found that the attitude of the obese person toward body and sexuality was the determining factor in the prediction of sexual functioning, establishing a directionality of the relationship between intimacy and body image (Areton, 2002; Schembri \& Evans, 2008). The recent study by Schembri \& Evans (2008) drew upon a sample of 225 women aged 18-63 to investigate the relationship of attachment, selfsilencing, and self-consciousness during sexual activity and bulimic symptoms. Self consciousness during sexual activity was the best predictor of bulimic symptoms, followed by anxious attachment. The researchers concluded that body image concerns (as measured by self-consciousness during sexual activity) were closely related $(p<0.01)$ to attachment (as measured by intimacy), and that targeting these relationship processes in therapy may result in more effective therapeutic outcomes for eating behaviors in women.

A Swedish study (Adolfsson et. al, 2004) reported a decrease in sexual activity and sexual satisfaction during periods of weight gain, and an increase during weight loss. 
Obesity, like sexuality, has been recognized to have a relational, homeostatic function. Attitude toward one's body and its involvement in sexual behavior, as a dimension of body image, has rarely been considered in terms of weight management efforts, while issues of intimacy and sexuality are often examined in relation to traditional eating disorders - bulimia nervosa and anorexia nervosa.

Obesity generates body image dissatisfaction - a factor in sexual avoidance (Jagstaidt et al., 1997). This has long been clinically demonstrated, however, the psychosomatic flow of events such that sexual avoidance generates body image dissatisfaction (and thus obesity) has not been indicated. Other studies suggest that psychological disturbances are a consequence rather than a cause of obesity (Friedman \& Brownell, 1995; Kolotkin et al., 2006).

Results from a 2006 study on obesity and sexual quality of life (Kolotkin et al., 2006 ) indicated that roughly one third of women (32.8\% to 37.6\%) reported that they usually or always did not enjoy sexual activity, had little sexual desire, experienced difficulty with sexual performance, and avoided sexual encounters because of their weight. Approximately one half of obese women (54.7\% to 61.2\%) reported difficulty with these aspects of sexual quality of life at least some of the time. Sexuality of the obese, as well as a consensus on its measurement, remains largely unexplored.

Freud had further postulated neuroticism above eroticism in the obese, where excess eating serves to compensate social avoidance and sexual inhibition as a result of an oral phase regression (Jagsdaidt et al., 2001). Those who perceive their bodies in negative terms are obviously more likely to be sexually avoidant. The balance between 
losses of control expressed in overeating, and over control through dieting has been found to serve as a behavioral pattern in subjects where relationships and sexual behavior are concerned (Jagsdaidt et al., 2001). Neither physicians nor patients are accurately aware of the topics that underlay obesity and excessive consumption of food, leaving the true psychological issues untreated and may explain poor results where long term weight control are concerned. Interestingly, bulimic women were found to experience orgasm with masturbation more frequently than with sexual intercourse. The study suggests bulimics are engaged in a struggle for control over their eating and body weight which is then generalized to other areas of their lives including sexual behavior. Where obesity is concerned, it has been suggested that this self-imposed physique serves as a distraction from other psycho-pathology (Jagsdaidt et al., 2001).

The preoccupation of body image for men has often been obscured by the field's focus on thinness, which, although previously accepted as appropriate for the study of women, may inadequately capture both men and women's body image preferences (Schooler \& Ward, 2006). The study reports puberty as the average onset age of body image dissatisfaction disorders. For young women, this stage of development brings with it a lesser freedom of activity, a loss of control over relationships and a strong emphasis on sexuality. For young men, this period brings about more freedom, responsibility and status, and a stronger emphasis on initiating relationships (Brumberg, 1988; Brumberg, 1997). This is an important consideration when examining body mass index in relation to gender, whereas body mass index tables do not reflect divisions in gender - yet another homogenous approach to a complex, heterogenous issue (“Statistics," 2006). 
The drive toward emaciation in young anorexics at a time of physical development first raised the possibility of an underlying fear of emerging sexuality. It is the linkage of weight with sexual development that was found to be the true object of fear (Crisp, 1980). Ultimately, self starvation is the patient's attempt to forestall the outward signs of sexuality. The same result of negating outward sexuality can be achieved through obesity in men and women; the physique becomes asexualized.

Researchers have found that the attitude of the obese person toward body and sexuality was a determining factor in the prediction of sexual functioning, establishing a relationship between intimacy and body image (Adolfsson, et al. 2004; Kolotkin et al., 2006). The study by Jagsdaidt et al. (2001) suggested that those who perceive their bodies in negative terms are more likely to be sexually avoidant, however, limited research examines the relationship of body mass index as it pertains to the ability to achieve intimacy. Fear of intimacy may reveal an important relationship with regard to body mass index.

\section{Body Image and Intimacy}

The majority of scientific literature on body image has focused on the evaluative (pertaining to body satisfaction) dimension of this model. The research has been focused upon young women and those with eating disorders, but rarely how the model relates to diverse populations in a sociocultural context (Cash \& Pruzinsky, 2002). In addition, the model underestimates the complexity of social relations that shape and are shaped by body image experiences. Wiederman's (2002) work illuminates the everyday observation that links women's sexuality and body image in a logical fashion - that poor body image 
will result in poor quality and frequency of intimacy - yet the topic has infrequently been studied, and rarely considered in men. The study confirmed the hypothesis that such body image self-consciousness was strongly correlated (mean inter-item correlation of .52) to less heterosexual experience and greater difficulty with sexual interaction (Wiederman, 2002).

Body image is closely related to the experience of romantic relationships and can affect both the quality and quantity of sexual and intimate experiences (Wiederman, 2002; Yamamiya, 2003, Cash et al., 2004). Internal (cognitive) models of adult attachment theories have long focused upon concepts of self-modeling and othermodeling (valuations), reflecting two basic dimensions of anxiety and avoidance of interaction (Cash et al., 2004). In this 2004 study, a three-step regression analysis examined anxious romantic attachment, general attachment security and general preoccupied attachment, with results accounting for $34.6 \%$ of variation in body image. Avoidant behavior has also been examined as an external, behavioral component of body image for both men and women; self-perceptions of social attractiveness were positively related to the intimacy achieved in interaction (Nezlek, 1999). Individuals who feel insecure, of, or in their interpersonal attachment - characterized by anxiety that manifests in avoidant behavior - may also exhibit insecurity about physical worth. For both sexes, greater body image dysfunction was correlated to less secure general attachment, including more preoccupied general attachment and more anxious romantic attachment (Cash et al., 2004). Body image disturbance has also been found to characterize individuals with eating disorders, and has been found to exist with greater prevalence in 
the overweight and obese (Cash et al., 2004; Cash, 1993; Cash, 1990; Rodin et al., 1985; Garner, 1997).

A recent study (Aubrey, 2007) suggested that body surveillance partially mediated the relation between exposure to sexually objectifying television and magazines and body shame, appearance anxiety, and body image self-consciousness during physical intimacy. One may deduce that such heightened surveillance of the body may result in avoidant behavior within the interpersonal realm. Further, heightened surveillance may result from non-conformity to social ideals (Aubrey, 2007).

An integrative review of literature by Newton et al. (2005) pertaining to romantic relationships and women with anorexia nervosa has highlighted the dearth of research in measuring and exploring the concepts of intimacy and disordered eating. The study revealed that women with anorexia nervosa who are coupled consistently experience lower levels of self-reported intimacy than couples without overt individual or relational issues. In addition, these women report improved intimacy with their respective partners as their treatment progressed and healthy body weight was restored. Analyses revealed statistically significant difference $(p<0.02)$ from treatment entry to program discharge (Newton et al., 2005). The review of literature fails to conclude that the deficiencies in intimacy experienced by women with anorexia are antecedents or consequences of their disordered eating. Such studies provide a starting point for intimacy related research for women with anorexia nervosa, but these findings may also speak to women who suffer from disordered eating and body image across a spectrum (Newton et al., 2005). 
Little research has been conducted to examine the relationship of body image avoidance and fear of intimacy, and even less as it relates to body weight (Cash et al., 2004). Of the little research pertaining to the relationship of body image and intimacy, Brennan and Shaver's (1995) landmark study found that every scale of the Eating Disorder Inventory (EDI; Garner, 1991), including the Body Dissatisfaction Scale (BDS), correlated with significance to women's preoccupied attachment in romantic relationships. No study prior to Cash et al.'s 2004 work on body image and interpersonal functioning had examined body image and attachment in males. Multiple regression analyses indicated romantic attachment as the strongest predictor of body image dysfunction for men and women, with insecure attachment correlated to a high degree of body image dysfunction (Cash et al., 2004). The results of the aforementioned study confirmed significant relationships between interpersonal functioning and body image.

Haines \& Neumark-Sztainer (2006) reported body dissatisfaction to be common among children and adolescents and considered how body image dissatisfaction and the avoidant behavior it spawns may be relevant in examining the development of obesity. Such avoidant behaviors involve isolation which entail lower levels of physical activity and sometimes - binge eating. A further connection toward this avoidant isolation among children and adolescents can be found later in life when adulthood assumes the development of intimate relationships, though not included in this study. Body image dissatisfaction is well established as a risk factor for the development of eating disorders [according to the Diagnostic and Statistical Manual of Mental Disorders (DSM IV) criteria] but has rarely been considered toward the incidence of overweight and obesity. Their reported findings from cross-sectional and prospective research suggest that 
interventions aimed at improving body satisfaction may have implications for the prevention of obesity and eating disorders among youth (Haines \& Neumark-Sztainer, 2006); the ensuing developmental sanctions imposed by avoidant isolation due to body image dissatisfaction may thus be thwarted.

Not only does the research conducted by Haines \& Neumark-Sztainer (2006) illuminate pathways toward the development of obesity, potential risk and protective factors are suggested toward further etiologic inquiry. Issues of self-esteem, interpersonal relationships, affect, and role modeling of weight-related behaviors and attitudes are addressed toward healthy eating and physical activity behaviors. Specifically, the research suggests preliminary evidence that issues of disturbed body image preceding adulthood may have relevance for the development of the spectrum of weight-related disorders.

The common sense approach dictates that looking one's best according to the norms of the society in which we live makes one more desirable - and our society almost overwhelmingly demands a slender figure - yet we continue to grow (Smuts, 1992; Nezlek, 1999; "Statistics,” 2006). Learned social behavior can modify sexual activity based upon the positive and negative attributes ascribed to body image that impact upon self-esteem (Strauss \& Wise, 1978), and weight bears significant social implications. Clinically obese women were found to be $37 \%$ more likely to have experienced major depression during the past year compared to average-weight women. Comparatively, for men, being underweight - not obese - was associated with increased risk for major depression. The suggestion is that weight bears psychosocial consequences that differ based upon gendered implications of weight (Carpenter, et. al, 2000). 
The present Western vogue for men includes broad shoulders and large biceps; young men are as likely to desire weight gain as weight loss (Drewnowski \& Yee, 1987; Silberstein et al., 1988). In line with their female counterpart, men who experience shame with regard to their bodies may be apprehensive about showcasing their bodies during sexual activity. However, as masculine sexual scripts in our culture prescribe that men be sex driven (Holland et al., 1990), this shame may not prevent engagement in sexual activity. Rather than withdrawing physically from sexual activity, men may remain engaged in sexual activity but may be emotionally withdrawn to create a safeguard and distance from any potential negative evaluations (Schooler \& Ward, 2006). This dynamic poses further issues for their respective partners; men's attitudes about women's bodies (as well as other men's bodies) may play a role in shaping body image dissatisfaction. As men learn about the male body ideal, media and sociocultural markers (Faith \& Schare, 1993) also transmit messages about the female body ideal. Media use has continued to endorse a thin ideal for women among both men and adolescent boys (Hargreaves \& Tiggemann, 2003; Harrison \& Cantor, 1997). Additionally, heterosexual men have been found to perceive average women and even their own romantic partners as less attractive after viewing images of women bearing the thin ideal (Kenrick \& Gutierres, 1980).

The Western relationship between obesity and sexuality is a surprisingly older phenomenon- usurped by the mass media to produce powerful images propagating the myth of "body beautiful", but oddly not involved in the treatment of obesity. Influenced by Freud's hyper-sexualized and often male-centered psychology, psychoanalysts of the 1920s and '30s reported eating as an erotic substitute, with the consumption of "dirty" foods in place of less acceptable lewd acts (Bess, 1997). Studies have reported links 
between sexuality and body dissatisfaction, dieting, and binge eating behavior for women, however, failing to speculate that sexuality and obesity hold a connection, nor how obesity may serve as an adaptive reaction to attitudinal psychopathology (Cash et al., 2004; Beach \& Martin, 1985).

As psychoanalytic theory has fallen out of vogue, obesity and weight control have been the subject matter of more behaviorally-based interventions (Beach \& Martin, 1985). Exhaustive searches through current literature in medical and psychological journals fail to reveal much insight into the relationship of obesity and sexuality.

An unpublished study conducted by Shipman and Schwartz at Michael Reise Hospital in Chicago (1976), but cited by Beller (1977), found that overweight women were more sexually appetitive than thinner women. The study hypothesized that adiposity served as a somatic metaphor for psychological armor that fat people employ as a defense against sex and love. According to this theory, the factors that were to have supported obesity should have also contributed to a lack of sexual appetite. This hypothesis was not supported by the results of the study, but supported a Freudian rationale that sexual interest is appetitive in nature, and co-exists with a need for holistic nurturance at the oral, anal and phallic stages of psychological development (Freud, 1962). While this rationale may best be examined within the realm of clinical psychology, the prevalence of overweight and obesity as a commonplace phenomenon make the Diagnostic \& Statistical Manual of Mental Disorders (DSM IV) unable to consider overweight and obesity as a disorder with psychological implications ("Statistics", 2006). Public Health may thus serve as an integrative discipline to further this new conceptual framework for understanding underlying incentives to achieve and maintain overweight \& obesity. 
The exploration of emotional issues that underlie obesity have been advocated by researchers toward effective treatment (Beach \& Martin, 1985). This may also explain, in part, the failure of community based interventions toward excessive weight since consumptive appetites are both subjective and personal in origin. They conclude that for women, food is a means of denying sexuality and is used to replace love, affection, and sexual desire (Beach \& Martin, 1985).

Scientific data has also found support for these correlates of consumptive need. Obesity has an impact on physical and emotional well being as well as psychosocial functioning, much as sexual satisfaction and intimacy are crucial in contributing to physical and emotional well being. A Swedish study (Adolfsson et. al, 2004) found that obesity and sexuality have purposeful and relational homeostatic functions. Eating and feeling physical satisfaction can offer gratification and mediate stress and distress, including feelings of anxiety, anger, sadness, sorrow, and loneliness. Oxytocin, a calmness hormone, is released by massage, sexual arousal, and orgasm just as it is released by the consumption of fat (Adolfsson et. al, 2004). Obese individuals have been expected to be less sexually interested, attractive, and capable of developing a satisfying romantic relationship than persons of normal weight. Changes in sexual interest and rate of sexual activity have been described coinciding with weight change - however it is premature to attribute these changes to biology or, instead, to socialized norms and expectancies (Adolfsson et. al, 2004; Carpenter, et. al, 2000; Cash, 1990). Changes in sexual interest and activity diminish when weight gain is experienced, and increase where weight loss is realized. These changes may be biologically innate or socialized based 
upon cultural contingencies - both yield a learned social behavior that can modify sexual activity (Areton, 2002; Strauss \& Wise, 1978).

In traditional societies, a prosperous socioeconomic status had been associated with being heavy-set. Oxford's English Dictionary (1989) defines "lean" as "wanting in flesh, innutritious" whereas "fat" is defined in desirable terms as "fertile, rich, abundant and plentiful". Contemporary culture and dialectics demand the inverse to be true (Smuts, 1992). Seventy-one percent of pre-industrial societies suffered shortages in food in at least one year of three. Accordingly, mothers suffered a caloric deficit at least once in the years between conception and weaning. A reflection of adaptive male mate preference in Darwinian terms suggests a preference for plump females in response to these conditions (Smuts, 1992). The adaptive model for a historical importance of fat storage on the part of women may offer insight as to the current cultural constructs of weight and body image in an era of medical birth control and alternative valuations of lifestyle. It may behoove us to remember that other cultures do not experience any of this to be true and, instead, consider large women to be the most beautiful (Ford and Beach, 1980; Brown, 1993). Ethnographic evidence indicates that of a total of 26 tribes from all over the inhabited globe who have ever indicated a preference in the matter, only 5 preferred their women slender (Smuts, 1992). To this small roster should now be added, presumably, the tastes of twentieth-century Americans and western Europeans (Beller, 1977).

Wiederman's (2002) work brought to light that poor body image would result in poor quality and frequency of intimacy - yet the topic has infrequently been studied, and rarely considered in men. No study prior to Cash et al.'s 2004 work on body image and interpersonal functioning had established such a strong relationship between romantic 
attachment and body image dysfunction for men and women, and it was also the first study of its kind to include men in the study sample. In addition, little literature is available that includes the treatment of body weight in this discussion. Beach \& Martin's 1985 study found that, for women, the use of food provided a means of denying sexuality, affection, and sexual desire. Two decades later, the focus of the 2004 study by Cash et al. examined insecure attachment correlated to a high degree of body image dysfunction; the study is one of few that have been conducted to examine the relationship of body image (measured by avoidant behavior) and intimacy (measured by avoidant behavior). This relationship is important, given the findings that reveal body weight (BMI) as being closely tied to body image (Cash et al., 2004), and body image as an important factor involved with intimacy.

\section{The Relationship of Gender}

J.B. Nezlek’s 1999 study “Body Image and Day-to-Day Social Interaction”, published in the Journal of Personality found that for both men and women, selfperceptions of social attractiveness were positively related to the intimacy they found in interaction. Further, while many studies such as Wiederman and Hurst's 1997 study “Physical Attractiveness, Body Image, And Women's Sexual Self-Schema" examine only female subjects, men are increasingly internalizing the socio-cultural mores about attractiveness (Cash et al., 2004).

Studies have long established a relationship between sexual orientation and eating psychopathology where homosexuality in men serves as a risk factor for the development of eating disorders, and serves as a protective factor in homosexual women (Heffernan, 
1994; Meyer, 2001). These findings have been extrapolated from, and built upon findings from studies on women only. Femininity has long been studied with regards to body image and its relationship with intimacy (Cash et al., 2004), mainly because research on the subject matter has been based upon female samples. Body image disturbance has been found to characterize individuals with eating disorders, and has been found to exist with greater prevalence in the overweight and obese - and mainly within female samples (Cash et al., 2004; Cash, 1993; Cash, 1990; Rodin et al., 1985; Garner, 1997). The identification of risk factors for the development of body image disturbance and eating disorders have gained an increasing recognition of gender roles and sexual preferences. To date, the analyses of these variables and their role in body image, intimacy and body mass index have been limited, and these factors have not been evaluated in a single study (Heffernan, 1994; Meyer, 2001). What has been indentified more clearly is that social constructs of masculinity and femininity hold protective and risk factors for the development of eating psychopathology (Meyer, 2001).

With the increased importance of thinness in the feminine role resulting from a western thin ideal, along is the increased incidence of eating and body image disturbance associated with femininity (Ginis, 2005; Mussap, 2007; Weaver \& Byers, 2006). This is highlighted by the study by Ginis (2005) which examined sex differences in body image change and its correlates over the course of a 12-week strength-training program for men and women. Significant body image improvements were found for both sexes $(p<0.05)$, however, the correlates of body image change varied between the sexes. The rationale for this was that women generally reported more body image dissatisfaction than men, providing them with greater opportunity for change. In the 2007 study by Mussap, the 
relationship between feminine gender role stress and disordered eating symptomatology in a sample of 124 women, aged 18 to 40 , was examined. Fear of being evaluated as unattractive correlated with dietary restraint, eating concern, as well as bingeing and purging behaviors. The results indicate that the stress of conforming to the feminine gender role is associated with a range of unhealthy body attitudes and behaviors (Mussap, 2007). In our fat-phobic society, where female self worth is so intimately tied to a slim figure and male self worth is tied to muscle mass and its inherent machismo, these biological differences have critical and distinctive emotional consequences (Beach \& Martin, 1985).

The mantra of "thin is in" is not without its reverse notion; obese individuals are judged to be unattractive and assumed to be sexually perverse in the most asexual of definitions (Smuts, 1992). Some psychological maladjustment and their medical treatments may result in weight gain including Schizophrenia - which is partially characterized by a lessened interest in forming intimate bonds and often physically characterized by obesity (Hein et al., 1998). Sexual affects, however, are often the result of weight gain, reinforced by a culture that poses a hostile climate toward obesity.

A recent study (Schembri \& Evans, 2008) drew upon a sample of 225 women aged 18-63 to investigate the relationship of attachment, self-silencing, and selfconsciousness during sexual activity and bulimic symptoms. Adverse relationship processes (defined as anxious attachment, self-silencing and self-consciousness during sexual activity) were significantly associated $(p<0.01)$ with bulimic symptoms. Self consciousness during sexual activity was the best predictor of bulimic symptoms, followed by anxious attachment. The study found that women with bulimic symptoms 
attempt to change themselves to meet the perceived expectations of their partners. Schembri \& Evans postulated that targeting these relationship processes in therapy may result in more effective therapeutic outcomes for eating behaviors.

The socio-sexual implications of obesity in North America are such that obese women often feel unable to lure sexual interaction (Brumberg, 1988). Manifested in highly subjective ways, it has been suggested that obese women will often find themselves in relationships with men who initiate intimacy and are unconditionally accepting, or will find themselves in relationships that do not challenge their sense of worth (Bess, 1997). Psychological research has indicated that some women gain weight in order to protect themselves from sexual harassment or even the lure of sexual activity whereas husbands promote weight gain in their partners to ensure fidelity (Smuts, 1992). Whatever the motive, the underlying conception is that fatness negates sex appeal.

Despite seemingly more lenient attitudes, obese men have far fewer opportunities for sexual intimacy (Bess, 1997). Men continue to act as sexual initiators which serve to foster male sexual sense of self-worth. Obese men share fears of rejection with their female counterparts, yielding little desire to initiate opportunities for rejection (Bess, 1997). The relationship of fear of intimacy, body image avoidance and body mass index may hold importance in understanding the underlying motives and possible resolve for the overweight and obese.

The quality of the American experience as portrayed by media has long imposed a need for thinness (Brumberg, 1988; Brumberg, 1997; Garner et al., 1980). Attaining a slender form for women became not only a fashion statement, but one of sexuality and 
modernity through the latter portion of the twentieth century (Brumberg, 1988). A tight physique was in line with the shedding of the tight, moralistic restrictions of the Victorian era and rigid gender roles.

Between the late 1950s and 1970s, the number of diet articles in popular women's magazines had virtually doubled (Garner et al., 1980). The absence of depicted physical ideals that resemble those naturally occurring within society, act as reinforcement for perceptions of body beautiful. Fashion and product advertisements have themselves become advertisements for the "thin is in" ideal. The absence of depicted physical ideals that resemble those naturally occurring within society act as reinforcement for our warped perceptions of body beautiful and body acceptable (Brumberg, 1988; Brumberg, 1997). Other publications that spoke to the era, including Alex Comfort's (1972) The Joy of Sex, explicitly stated that "Fatness in our culture is unlovely. If you are grossly overweight, set about losing it, whether you value your sex life or only your life" (p. 245).

The sexual revolution and feminist movement changed the constructs of masculinity and femininity from traditionally rigid terms, allowing for a greater shift in gender roles which has continued to evolve over the past several decades (Brumberg, 1988). Feminist theorists support this concept that male-driven industries including media, advertising, cosmetics, etc. conspire to control and contain women's power and to profit from an industry created to promote dissatisfaction. Notable and outspoken advocates for this movement such as Naomi Wolf and Jean Kilbourne suggest that these industries manufacture anxiety about weight and appearance as a backlash toward the 
feminist movement (Brumberg, 1988). Conversely, little has been said about the same industries now targeting men in much the same manner.

NHANES data confirms that the percent of obese adults varied little from 1960 to 1980, but has increased considerably thereafter. Between 1980 and 2000, the percentage of obese males increased from $13 \%$ to $28 \%$ and from $17 \%$ to $34 \%$ for women ("Statistics," 2006). This era reflects the aforementioned shifts in gender and sex roles in our modern history following the sexual revolution and women's liberation movement (Brumberg, 1988). In addition, new media has enabled new means of communicating ideas and ideals. Intimacy has rarely been more exposed; this exposition of sexuality is threatening as it is alluring. Diets reflect these changes, marketed mainly to womenoffering a new body that will be admired by those around her and that by reducing one's physical size is also reflected by an apparent loss of all internal emotional conflict (Beach \& Martin, 1985).

In recognizing the complex issues that may serve to contribute to the rising incidence of overweight and obesity, modern technologies serve as a means to this end. Researchers have examined the degree to which the new media - including internet serves as an addiction and promotes personal isolation. Its use and access serve to reinforce cultural tastes, gender roles and additional opportunities for media consumption (Stern, 2001). An online survey of internet users suggests that sexual pursuits, ranging from visiting web sites with sexual themes to intense on-line sexual interactions may be the most common use of the internet (Stern, 2001). While sexually-themed internet sites are generally self-selected and pursued, the abundant use of sex in advertising has been steadily increasing since the early 1980s (Stern, 2001). Advertisers often use sexually 
attractive models in order to build associations between sex and their products; such images abound the cultural landscape. Interestingly, between 1979 and 1989, during which the AIDS epidemic came to light, explicit sexuality increased in print advertising and on primetime television (Stern, 2001). One may wonder if AIDS had opened the discourse for sexual behavior in this public manner, or if it had resulted in a safer sublimation of sorts via increasingly expressive and interactive media such as television and internet.

The internet is very accessible, making sexual material available to more people. Surveys suggest that one in four children who use the internet inadvertently find pornography (Lips, 2001; Stern, 2001). Of course, with the availability of telephone dialup services, pornographic videotapes, and cable television, the advent of the internet does not mark the first time that pornography has been introduced to the home (Stern, 2001). Despite the proliferation of media providing access to sexual material, it is uncommon to find images of obese couples, personal advertisements, nor adult erotic prints and movies that depict women with a body mass index of above 25 (Areton, 2002). In support of the dominant thin ideal and its role in contributing to, and perpetuating body dissatisfaction are content analyses of media such as magazines and television (Thompson et al., 1999).With more sexual and pornographic material available in forms from mass advertising to internet pornography, comes the greater opportunity for anonymous interaction (Stern, 2001). Constraints of weight bear little relevance, continuing a cycle of social and romantic isolation.

Media and culture have evolved greatly since 1980, along with the prevalence of overweight and obesity ("Statistics," 2006). Research and practice have moved toward 
considering gender in their study of body image, intimacy and body mass index, but despite exhaustive searches, these findings are limited and fragmented. The 1999 study by Nezlek, "Body Image and Day-to-Day Social Interaction”, found that for both men and women, self-perceptions of attractiveness were positively related to the intimacy they found in interaction. Many studies in the area have examined only female subjects, while men are increasingly internalizing the socio-cultural mores about attractiveness (Cash et al., 2004), and are becoming the larger cohort of obese Americans as compared to women. The implications of gender differences should be accounted for in gaining a better understanding of the relationship of body image and intimacy, and how behavioral manifestations of these may be related to body mass index.

Body Images: Representations of Race, Culture and Ethnicity

Obesity and a status of overweight are complex and chronic conditions resulting from a variety of factors. Ultimately, obesity is a direct result of an energy imbalance between an excessive caloric intake versus the amount of calories burned. When calories consumed are greater than calories burned, weight gain ensues. Body weight and individual homeostatic levels vary based upon genetics and metabolism, but are also impacted by behavior, environment, culture and socioeconomic status.

Obesity is often regarded as a disease of comfort. Physical inactivity, imbalanced diet, smoking and excessive alcohol consumption-which result from progress in civilization toward electricity, machinery, automation, energy dense foods, modern life and a globalized economy — are regarded as contributing factors (Choi et al., 2005). Ironically, in America, there is a disproportionate prevalence of overweight and obesity 
correlated with low income ("Statistics", 2006). Obesity results in health consequences including, but not limited to, cardiovascular disease, cancers, repertory disease, mental disorders, diabetes, etc. Behavior and environment are largely believed to be contributing factors to the current obesity epidemic, and targeted as the greatest areas for prevention and treatment. The most effective weight loss programs, however, have empirically combined diet, exercise and behavioral modifications; however, even at best these programs have had only moderate success (Lang, 2006). It is thus important to consider the underlying cues toward overindulgence in food, community and ethnic rituals where food is concerned, the nutritive quality of foods, as well as the underlying cognitive impetus for consumption which is variant upon individual, community, etc.

Adoption, twin, and family studies have consistently shown that obesity has a strong heritable component, with approximately $30 \%-70 \%$ of variability in body weight or fat mass being genetically determined (Devlin et al., 2000). While this is considered a major genetic effect, it leaves a large component of flux in body weight unexplained by genetic mechanisms further failing to explain the recent epidemic prevalence of overweight and obesity when considering that the genetic composition of the population does not rapidly change (Choi et al., 2005).

When considering these genetic mechanisms, it is important to note that according to the National Health and Nutrition Survey (NHANES), the age-adjusted prevalence of overweight and obese combined is not consistent across racial and ethnic divides. The anxieties toward a status of overweight and obesity in non-conformity of cultural taste toward thinness seem less troubling for black women when assessing physique and notions of "body beautiful" (Hebl, 2004). For example in the African American 
community, following decades of exalting that "black is beautiful", parents, educators and role models have set the example, encouraged and instilled a sentiment of selfrespect and esteem in young girls that defies the suggested cultural norm. It is thought that the above-mentioned flexible and broad conceptions of beauty held by AfricanAmerican and other minority groups enable an exertion of greater pride, control and agency in self-image that counteracts an oppressive message of conformity and uniformity (Brown, 1993; Fitzgibbon et al., 2000; Parker et al., 1995).

Permissive, positive valuations from peer groups, family and media furthered by a lack of community resources to actively promote nutritive eating and physical activity form an in-group that reinforce these attitudes and beliefs where weight is concerned. Empirical research finds that heavy-set African American women feel a greater confidence in their physique and often become more viable in the sexual marketplace (Lovejoy, 2001). A study of African American women representing a variety of different age-groups and class backgrounds found that approximately 40 percent of these women who were moderately to severely overweight by medical standards considered their bodies to be attractive or very attractive (Kumanyika, et al., 1993). The point to be made is not that African American women demonstrate a healthier attitude toward body image and food consumption; the explanatory models and incentives in cultural differences of gender roles may point toward a more effective treatment of overweight and obesity within this community and in our society at large.

The National Health and Nutrition Survey (NHANES) finds that the age-adjusted prevalence of overweight and obese combined in racial and ethnic minorities is generally higher than in Caucasians within the US (“Statistics," 2006) - highest among non- 
Hispanic black women (77.3\% prevalence) and there is additional research (Hebl, 2004) Kumanyika, et al., 1993; Lovejoy, 1991; Wilcox, 1990) to suggest that the anxieties of body weight seem less troubling for non-Hispanic black women when assessing physique and notions of body beautiful. The gender roles of black women differ substantially from their white counterparts, holding a role expectation of strength, independence and labor force participation (Wilcox, 1990), and thus carry greater esteem making them less susceptible to a white 'norm' for body as portrayed by media and cultural ideals.

Comparatively, white women are far more negative in their attitudes toward their bodies and are likely to feel overweight when their weight is within normal range (Lovejoy, 2001). Little evidence exists in how ethnic and racial backgrounds affect men to this same extent (Hebl, 2004).

The differences between black and white women in how they see their bodies (perceptual body image) and in how they feel about their bodies (attitudinal body image) form important consideration in research. Divergent ways of defining samples, inconsistent methods of measuring and categorizing weight status, and the use of differing body image measurements have contributed to problems in conclusive research, but also in the generalization of body image methodology and implications (Fitzgibbon et al., 2000).

Fitzgibbon et al.'s (2000) study indicates differences in the BMI point at which body image discrepancy occurs and the rate of increase of the discrepancy. The study also further identified the correlation that white women experience a body image discrepancy at lower weights than women who comprise minority populations. The 
majority of research in this area, however, has not addressed this issue of body image in a stratified multi-ethnic population, nor has research established baselines for these findings (Fitzgibbon et al., 2000). The study also highlights the difficulties in body image research as it pertains to race and ethnicity due to factors including acculturation, regional and generational differences, etc.

Other studies have also noted difficulties with research in regards to body image, race and ethnicity; Cafri et al. (2005)'s meta analytic study on body image found that ethnicity was not a statistically significant moderating effect for body image. The authors instead advocated for further inquiry into the influence of sociocultural factors on body image. In fact, Rosen et al. (1991), whose research resulted in the Body Image Avoidance questionnaire used in this study caution to avoid the treatment of race and ethnicity when examining body image avoidance due to the aforementioned complexities highlighted by Fitzgibbon et al. (2000). None of this is to suggest that racial groups do not have significantly different views of body image; the aim of this study is not to examine how these views differ between groups, but how perceptions of body image may be correlated to fear of intimacy and body mass index, as well as to examine gendered differences.

For Hispanic families, very traditional gender roles may also explain, in part, differences between gender groups. Machismo refers to a male role expectation for physical strength, authority and fearlessness (de Rios, 2001; Falicov, 1998; Vega, 1990). The complementary role for women - Marianismo - describes the woman's role as selfsacrificing, religious, responsible for running the household, and raising and bearing children (Bean, Perry \& Bedell, 2001; Falicov, 1998; Vega 1990). There is substantial 
research (Kumanyika, et al., 1993; Lovejoy, 1991; Vander Wal, 2004; Wilcox, 1990) to suggest that the anxieties of body weight seem less troubling for non-Hispanic black women and for Hispanic women when assessing physique and notions of body beautiful due to differences in gender role expectation.

The issues associated with negative body image have also received substantial attention with regards to gender, social status and cultural differences. This is appropriate given that, within Western cultures, there are unrelenting pressures with regard to body image ideals. Large-sample survey research suggests that $52 \%$ of men and $66 \%$ of women in America are dissatisfied with their weight (Garner, 1997), and some women report being on a weight-loss diet even when their weight is at or below normal (Rodin et al., 1985). Although a substantial percentage of men (88\%) who are dissatisfied with their weight do desire to lose weight, $22 \%$ of men who express dissatisfaction with their bodies actually wish to gain weight (Garner, 1997), most likely to meet the muscular ideals that are portrayed for men's bodies (Drewnowski \& Yee, 1987).

It would seem reasonable to propose that the pervasiveness of the thin body ideals would have an even greater impact on individuals who are, in fact, overweight or focused upon their body weight. As the social pressures for thinness and the associated stigmatization of obesity (Friedman, 2002; Fitzgibbon et al., 2000, Friedman \& Brownell, 1995) have increased, so has the rate of obesity. The rate of obesity has more than doubled since 1900 , and $64.5 \%$ of the adult population of the United States is classified as overweight or obese ("Statistics," 2006). Thus, among the overweight and obese segments of the population, as the pressure for thinness increases, the discrepancy 
between desirable body weight and actual body weight increases as well (Adolfsson et. al, 2004; Friedman \& Brownell, 1995). This would serve to intensify the body-image concerns of those who are objectively overweight.

Research suggests that depending on social class membership, there are either opportunities or impediments to engage in lifestyle activities consistent with weight control (Brown, 1993; Brownell, 1995; Fitzgibbon et al., 2000, Smuts, 1992). These considerations are important when assessing what are considered to be minority and disadvantaged groups in our society - including the obese. Thus, differences in obesity may be related to differences in body image, and the weight at which the differences begin to occur may explain, in part, differences in obesity between ethnic groups (Fitzgibbon et al., 2000).

The differences between black and white women in how they see their bodies (perceptual body image) and in how they feel about their bodies (attitudinal body image) form important consideration in research and implementation of preventative and treatment programs. Divergent ways of defining samples, inconsistent methods of measuring and categorizing weight status, and the use of differing body image measurements have contributed to problems in conclusive research, but also in the generalization of body image methodology and implications (Fitzgibbon et al., 2000).

Limitations of the current methods of examining body image are important; acculturation and increasing fragmentation of North American society through media and communication pose implications toward the validity and longevity of research in the 
area. Body image is a multidimensional construct that includes perceptual, attitudinal (Friedman \& Brownell, 1995), and behavioral components, and few studies bare the methodology that can accurately reflect our heterogeneous population - between race, ethnicity and gender - and within their subgroups (Fitzgibbon et al., 2000). It is important that the intended audience of any research in the area of body image is made aware of these inconsistencies and resulting margins of error and inaccuracy.

The Relationship of Fear of Intimacy, Body Image Avoidance and Body Mass Index Health behavior interventions within the realm of public health and beyond have empirically examined behavior at the individual level, and these initiatives seem appropriate where obesity is concerned. The theories that have driven this approach include the health belief model (Hochbaum, 1958; Rosenstock, 1960, 1966, 1974), the theory of reasoned action (Fishbein \& Ajzen, 1975), and the transtheoretical model (Prochaska \& DiClemente, 1983). Further direction in the field has sought to examine individual behavior in the built environment, in considering predisposing, reinforcing and enabling factors - and obesity has appropriately been examined in this manner. New paradigms are needed that address issues of public health from a multidisciplinary perspective so as to effectively modify behavior and maintain these changes.

The field of body image has experienced tremendous growth in the last 50 years, particularly as women's studies and media cultivation theories have evolved in the past twenty years (Areton, 2002). In the case of body image, the increase in the number of new and revised measures purporting to assess one or more of the multiple dimensions of 
body image has been well documented (Cash, 2002; Thompson et al., 1999). There is also great confusion and concern about the assessment considerations in this area from a variety of vantage points, and due to the multiple dimensions of body image (Thompson, 2004). Many of the available measures focus on size or weight, and their validity has been called into question as cultural, racial and ethnic interpretations of weight, size and body image are highly variable and continue to evolve (Fitzgibbon et al., 2000).

A new measure for body image was introduced in 2000, named the body image assessment for obesity (BIA-O) and was tested for reliability and validity in a sample of 1209 Caucasian and African American adult men and women (Fitzgibbon et al., 2000). While the reliability of the BIA-O was supported by test-retest reliability coefficients which ranged from 0.65 to 0.93 , the test has not been found appropriate for use in a nonobese sample. In addition, Fitzgibbon et al. (2000) highlight limitations in the use of this measure for African Americans, since this group is hetereogeneous in its composition based upon place of origin and generational differences - factors not addressed by the study’s methodology. The Body Image Avoidance questionnaire (Rosen et al., 1991), was selected for use in this study as it has been the most widely used and respected; it is also the only one to provide data on its reliability through internal consistency and retest reliability (Thompson et al., 1999).

Despite numerous measures of facets of the body image construct, no single assessment broadly measures a continuum of body image disturbance (Cash et al., 2004). The Body Image Disturbance Questionnaire (BIDQ), introduced in 2004, was derived from the Body Dysmorphic Disorder Questionnaire for use with non-clinical samples. 
Participants were 220 college women and 75 college men who completed an online survey containing this new assessment and established measures of body image and psychosocial functioning. Results confirmed that the Body Image Disturbance Questionnaire was internally consistent. Scores on this assessment also predicted psychosocial functioning above and beyond body dissatisfaction as a predictor, but limitations of this study include the small male sample size and racial heterogeneity (Cash et al, 2004).

Often, body dissatisfaction is regarded as an indicator of body image, when it should be viewed as a correlate, symptom, or risk factor of some dimension of body disturbance (Thompson, 2004). Much of the research to date has evaluated dissatisfaction, but conceivably, internalization may predict other dimensions of body image, such as avoidant behavior. Scales that measure avoidant behaviors resulting from body image and from intimacy are few (Thompson et al., 1999).

The Miller Social Intimacy Scale (MSIS), developed by Miller and Lefcourt in 1982 was considered for use in the present study. This popular instrument in the research of intimacy has been rated as very reliable (Lips, 2001) and was developed to fill the void of adequate intimacy scales. The scale asks participants to complete the questions thinking of their most intimate relationship in an attempt to measure a person's maximum level of intimacy (Miller \& Lefcourt, 1982). This is achieved through questions such as "How often do you show him/her affection?" or "How often do you confide very personal information to him/her?" 
The Intimacy Attitude Scale was originally developed by Amidon and was revised in 1983. The Cronbach alpha measure of internal consistency showed alpha values between .78 and .86 in five different groups. The test-retest correlation was .57 which improved to .84 after exclusion of three extreme scores and it was concluded that the revised Intimacy Attitude Scale is a reliable instrument with some degree of validity for use in research, diagnosis, and training.

The Fear of Intimacy Scale (FOI), developed by Descutner and Thelen (1991) was based upon the MSIS and used to assess the degree of inhibition of an individual's capacity to exchange thoughts and feelings of personal significance with another highly valued individual. Due to the greater range of interpersonal intimacy assessed by the Fear of Intimacy Scale, and in an effort not to exclude potential participants who may not have experienced an intimate relationship (as posed by the MSIS). Descutner and Thelen have shown the scale to be a valid and reliable measure of individuals' anxiety about close dating relationships, bearing a high validity in college-age samples and have reported a test-retest reliability for the scale of .89 and a coefficient alpha of .93 .

The dearth of scientific literature pertaining to the relationship of fear of intimacy, body image avoidance and body mass index fails to provide insight into dimensions of this relationship that may be relevant to understanding the epidemic of obesity in relation to social changes in our culture. Social relations both shape and are shaped by body image experiences, much as they are by experiences of intimacy - the latter has evolved alongside gender roles and modes of modern communication. 
The biological, evidence-based and less scientific psychoanalytic examinations of overeating as behavioral functions of emotional symptoms may provide a substantive rationale toward weight management (Beach \& Martin, 1985). Cues toward overindulgence and the North American taste for instant gratification are paramount and preferred. Neither physicians nor patients are accurately aware of the topics that underlay obesity and excessive consumption of food, leaving the true psychological issues untreated and may explain poor results where long term weight control are concerned (Beach \& Martin, 1985). Furthermore, public health campaigns also target surface behaviors and do not explore the pathway models that underlie overeating and physical inactivity. Gender roles and expectations may help to explain differences in behaviors, including those related to body image and intimacy that result in outcomes associated with body mass index.

The 2004 work on body image and interpersonal functioning (Cash et al., 2004) was the first to examine how these psychological components are gendered and offers opportunities to consider how body mass index may be involved in this relationship of psychosocial health. Recent contributions in communication and psychological theory contend that an objectifying culture can create a heightened level of body selfconsciousness and may mediate the relationship of negative body emotions and sexual dysfunction (Aubrey, 2007). This survey of 384 undergraduates suggested that body surveillance partially mediated the relation between exposure to sexually objectifying television and magazines and body shame, appearance anxiety, and body image selfconsciousness during physical intimacy. Surprisingly few gender differences were found, 
and offered some insight into how body image may underlie avoidant behaviors toward interpersonal functioning and uses of the body (Aubrey, 2007).

The Body Mass Index scale has been used by the World Health Organization as the standard for recording obesity statistics since the early 1980s as a reliable measure (“Statistics," 2006). Because the BMI is dependent only upon net weight and height, it makes simplistic assumptions about distribution of muscle and bone mass, and thus may overestimate adiposity on those with more lean body mass (e.g. athletes) while underestimating adiposity on those with less lean body mass (e.g. the elderly). Despite this, BMI categories are regularly regarded as an acceptable tool for measuring whether sedentary individuals are "underweight," "overweight" or "obese” ("Statistics,” 2006) and have been used accordingly in this study.

The focus of this research will examine the relationship of fear of intimacy, body image avoidance and body mass index, as well as how this relationship is gendered to suggest how the interrelation of these variables may result in behaviors leading to the increasing prevalence of overweight and obesity in our society. The following research questions are posed and hypotheses suggested. 
Question \#1: To determine whether a relationship exists between body mass index and fear of intimacy.

Question \#2: To determine whether a relationship between body mass index and fear of intimacy varies by gender.

Question \#3: To determine whether a relationship exists between body image avoidance and fear of intimacy.

Question \#4: To determine whether a relationship between body image avoidance and fear of intimacy varies by gender.

Question \#5: To determine whether a relationship exists between body mass index and body image avoidance.

Question \#6: To determine whether a relationship between body mass index and body image avoidance varies by gender.

Hypothesis \#1: As body mass index increases, fear of intimacy increases.

Hypothesis \#2: The relationship of body mass index and fear of intimacy varies by gender.

Hypothesis \#3: As body image avoidance increases, fear of intimacy increases.

Hypothesis \#4: The relationship of body image avoidance and fear of intimacy varies by gender.

Hypothesis \#5: As body mass index increases, body image avoidance increases.

Hypothesis \#6: The relationship of body mass index and body image avoidance varies by gender.

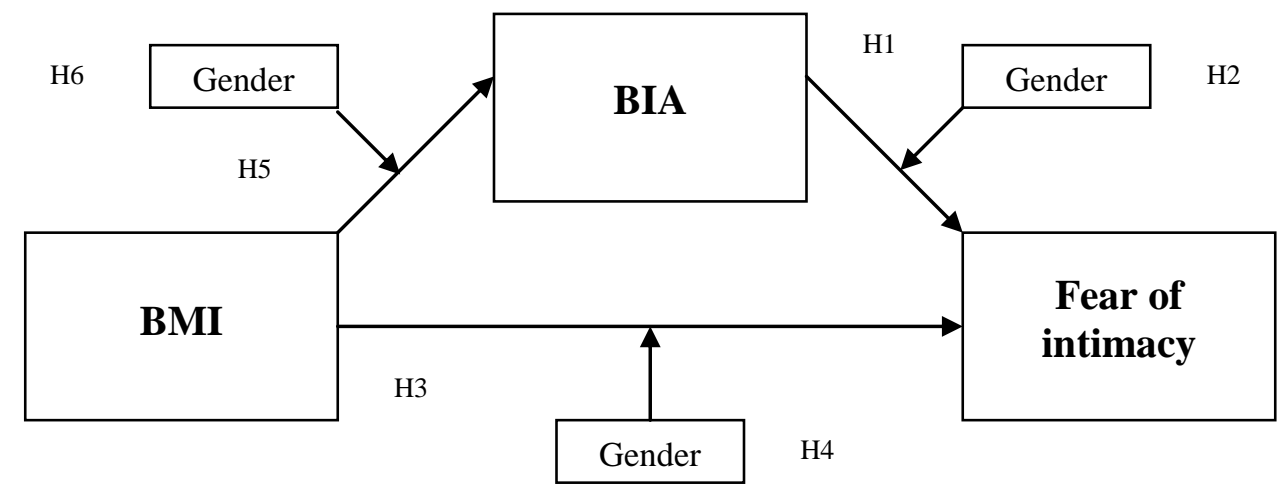


The following tools will be used to provide data for the testing and analysis of these hypotheses - the Body Image Avoidance (BIA) questionnaire (Rosen et al., 1991) and the Fear of Intimacy (FOI) scale (Descutner \& Thelen, 1991), as well as Body Mass Index (BMI).

The Fear of Intimacy scale (FOI) developed by Descutner \& Thelen (1991) was selected for use in this study. There are few measures of intimacy, and independent studies by other researchers have shown the Fear-of-Intimacy Scale (FOI) to be a valid and reliable measure, with a high internal consistency and test-retest reliability (Thompson, 2004). Measures of intimacy are often subject to criticism as being heterosexist in nature; the FOI scale is one of few that have been tested on a sample of 40 lesbians and 57 gay men (Greenfield \& Thelen, 1997). The FOI scale showed a high internal consistency when reviewed by Greenfield \& Thelen in 1997. Overall, the FIS was shown to be a valid, reliable, and useful measure of fear of intimacy for heterosexual and homosexual populations. Construct validity was established by factor analysis and significant correlations, and Descutner \& Thelen (1991) provide strong evidence that the scale bears a high validity in college-age samples and have reported a test-retest reliability for the scale of .89 and a coefficient alpha of .93 .

The Body Image Avoidance questionnaire (Rosen et al., 1991), created to measure the behavioral dimension of body image disturbance such as the avoidance of intimacy was most appropriate for use. Much of the research to date that has resulted in the development of measurement tools have evaluated dissatisfaction, but conceivably, internalization may predict other dimensions of body image, such as avoidant behavior 
(Thompson, 2004). Of the few behavioral measures, the Body Image Avoidance questionnaire has been the most widely used and respected; it is also the only to provide data on its reliability through internal consistency and retest reliability (Thompson et al., 1999). Rosen et al. (1991) provided multiple sources of validity data, including convergence with established measures of body-image disturbance. The Body Image Avoidance questionnaire was selected to determine convergent validity to complement measures of body mass index and intimacy. 


\section{CHAPTER III}

\section{METHODS}

This study was approved by the Institutional Review Board at Florida International University. The research used a cross-sectional, point-prevalence design. The data method included a validated, closed-ended survey instrument designed to measure the variables that are being examined in this study.

The survey instrument consisted of two scales that are germane to the variables being examined. They were (1) Descutner and Thelen of the University of Missouri's (1991) Fear-of-Intimacy scale and (2) Rosen, Srebnik, Saltzberg, and Wendt's (1991) Body Image Avoidance Questionnaire which deals with concrete behaviors that reflect one's belief about personal attractiveness. The researcher also gathered self-reported gender, height and weight data from subjects to determine body mass index (BMI). Age has been found to be an important factor in classifying weight, height, BMI, and overweight from self-reports. An analysis of NHANES III data indicated statistically significant differences for the mean error (measured-self-reported values) for height and weight that were notably lower for younger $(<60)$ age groups (Kuczmarski et al., 2001). This is further support for the study sample to have consisted of participants aged 18 to 34.

A convenience sample was conducted to reflect a university population of men and women, aged 18-34 taken from Florida International University's Biscayne Bay Campus. Although race and ethnicity are clearly involved in discussions of culture, environment and socioeconomic trends, they have not been treated as variables for the 
purpose of this study. Race and ethnicity were not criteria for the purpose of this study since these are subjective terms; they have been shown to serve as confounders in a priori research where body image is concerned due to factors including acculturation, regional and generational differences (Fitzgibbon et al., 2000). According to the review article by Fitzgibbon et al. (2000), the majority of research in this area has not addressed the issue of body image in a stratified multi-ethnic population, nor has research established baselines for these findings. It is important, however, to consider race and ethnicity within the framework of social psychology and that multiculturalism bears implications toward intimate social interaction (Nezlek, 1999; Fitzgibbon et al., 2000; Choi et al., 2005). Instead, the methodology employed aimed to gather a racially and ethnically diverse sample population which was reflected in the data.

\section{Participants}

The population of interest consisted of men and women taken from Florida International University's Biscayne Bay and University Park. A total of 113 participants were included in the study. Of this total $n$, there were 56 males included and 58 females, surpassing the targeted sample size of 100 subjects as determined by a prospective power analysis, based upon a desired medium effect size $(r=.30)$ with a one-tailed alpha of .05 . Based upon this same prospective power analysis, data analysis was split by gender and would have required a minimum of 40 respondents per gender (Cohen, 1988).

Cooperative agreements were made with professors at Florida International University to recruit student subjects from classes at the Biscayne Bay Campus, as well as the University Park Campus. Inclusion criteria consisted of: a) men and women aged 
18-34; and b) fluency in English, required tocomplete the questionnaire battery. Participants who were cognitively impaired, as evident by an inability to comprehend instruction, were excluded since this condition may have compromised the participant's ability to comprehend and participate in the assessment processes.

\section{Procedures}

Cooperative agreements were made with professors at Florida International University to recruit student subjects from classes at the Biscayne Bay Campus, as well as the University Park Campus. Using different classes from different academic programs and campus locations provided a sufficient number of participants to gather intake data in a timely manner, and increased the likelihood that results would be representative and not related to idiosyncrasies associated with one student population involved in a particular academic program. Classes were assessed at the beginning of the fall 2007 school semester to determine reflective heterogeneity therein to obtain a broad range of subjects fulfilling the selection criteria.

Potential participants were presented with the opportunity to participate in the study during regularly scheduled class time as per the cooperation of willing faculty. Interested participants who met screening criteria were issued informed consent forms (approved by the Florida International University Institutional Review Board) and were given a separate package containing the survey instruments and data form to be completed. The researcher administered all packages and documents of informed consent. To protect subject anonymity and confidentiality, the only personal information included with the completed questionnaires was a query of age and gender. Consent forms were 
collected separately from questionnaire packages. Questionnaire and consent packages were numbered, and in total 125 packages were distributed of which 113 fully completed packages were returned. A total of 55 male respondents' questionnaires and 58 female respondents' questionnaires were obtained. Half of the questionnaire packages presented the Body Image Avoidance Questionnaire preceding the Fear of Intimacy Scale - the other half were reversed in order to ensure proportionately stratified sampling and to avoid a leading bias in the collected data. This method is consistent for this area of research (Thompson, 2004).

\section{Measures}

The survey instrument consisted of two scales that are germane to the variables being examined. The selection of these scales was based upon their highest caliber of validity and reliability in their respective areas as evident though a meta-analysis of the literature (Wiederman, 2000).

The Fear-of-Intimacy Scale (FOI), developed by Descutner and Thelen of the University of Missouri (1991) was used to measure comfort with intimacy. The scale consists of two parts - the first contains 30 statements that require respondents to imagine themselves in a close dating relationship and to indicate how strongly they agree or disagree with each statement on a five-point scale. The second part consists of five statements pertaining to their past relationships that respondents rate on a five-point scale. Descutner and Thelen have shown the scale to be a valid and reliable measure of individuals' anxiety about close dating relationships. Descutner and Thelen (1991) provide strong evidence that the scale bears a high validity in college-age samples and 
have reported a test-retest reliability for the scale of .89 and a coefficient alpha of .93. A high score on the FOI scale indicates lessened fear of intimate relationships.

Rosen, Srebnik, Saltzberg, and Wendt's (1991) Body Image Avoidance

Questionnaire (BIA) deals with concrete behaviors that reflect one's belief about personal attractiveness (rather than relying on the subject's subjective description of their beliefs and emotions). This may then serve toward a more objective, reliable way of measuring this variable. A sample item is "I do not go out socially if the people I am with are thinner than me." The 19-item questionnaire uses a 6-point likert scale (ranging from $1=$ Never to $6=$ Always). Scores range from 0 to 76 , with higher scores indicating a greater tendency to avoid social activities in which body weight and appearance may be a focus. Rosen et al. (1991) reported an internal consistency coefficient of .89 and a test-retest correlation of .87 across two weeks. Rosen et al. (1991) also provided multiple sources of validity data, including convergence with established measures of body-image disturbance and disordered eating and notable changes in scale scores after treatment for body-image concerns.

Lastly, the Body Mass Index (BMI) score was considered. The BMI scale has been used by the World Health Organization as the standard for recording obesity statistics since the early 1980 s as a reliable measure ("Statistics," 2006). The researcher also selected the sample population of ages 18 to 34 such that this relatively small range in ages would exclude variances in BMI that may be related to age and health status (e.g. the elderly), as well as in seeking a heterogeneous sample according to WHO guidelines for BMI ("Statistics," 2006). 
The researcher requested self-reported height and weight on the final page of the questionnaire package to determine body mass index scores using the formula:

$$
\mathrm{BMI}=\text { weight }(\mathrm{lb}) /[\text { height }(\text { in })]^{2} \times 703
$$

Results were then recorded as a Body Mass Index result to reflect the following standard BMI chart as used by the Centers for Disease Control and Prevention:

\begin{tabular}{|c|c|}
\hline BMI & Weight Status \\
\hline Below 18.5 & Underweight \\
\hline $18.5-24.9$ & Normal \\
\hline $25.0-29.9$ & Overweight \\
\hline 30.0 and Above & Obese \\
\hline
\end{tabular}

The rationale in requesting self reported height and weight following the scales was to avoid further biases of body weight when responding to questions of body image avoidance and intimate interaction. It was anticipated the self-reporting would yield inaccuracies to actual body weight (Flegal, 2005), however, the self-concept of weight and its relation to the scales is inherent to the relationships that are of interest (Cash \& Pruzinsky, 2002). Finally, this method is consistent with regards to research in the area of body mass index and body image (Cash \& Pruzinsky, 2002; Cash et al., 2004). 


\section{Statistical Analysis}

A total number of 113 participants included 56 men and 58 women, aged 18 to 34. Sample size was determined by a prospective power analysis (G*Power, v.3), based upon a desired medium effect size $(r=.30)$ with a one-tailed alpha of .05 (Cohen, 1988; Erdfelder et al., 1996). Considering such criteria, a minimum of only 80 subjects were required to obtain the smallest acceptable effect size of .30 with statistical power of .87 (Lipsey, 1990; Cohen, 1988). Further, of this minimum total 80 subjects, equal representations of genders were required since the study at hand aims to examine gender differences. As a result, additional subjects were gathered to further support the power of the study and hypotheses to be tested. These numbers of participants are consistent with studies in the field of body image that have also employed correlational measures to measure body image (Cash et al., 2004; Wiederman, 2000).

For hypotheses $1,3 \& 5$, which sought to examine correlations between scores for fear of intimacy, body image avoidance and BMI, overall, the prospective power analyses computed provided the smallest acceptable effect size of .30 with statistical power of .87 (Lipsey, 1990; Cohen, 1988) at only 80 subjects. For hypotheses 2, 4 \& 6, which examined gendered differences for the aforementioned variables, Fisher's R-to-Z transformation provided a consistent effect size with only 40 subjects per gender group (Lipsey, 1990; Cohen, 1988).

The data analysis for this research was conducted using SAS (v.9.1) software. Two statistical procedures will be used to analyze the data.

First for hypotheses $1,3 \& 5$, Pearson correlations between scores for fear of intimacy, body image avoidance, and BMI, overall and for men and women separately 
were calculated and tested. Second, for hypotheses $2,4, \& 6$, the difference between the Pearson's correlations for men and women were tested with a Z test using Fisher's r-to-Z transformation using VassarStats. All tests were declared significant at $\mathrm{p}<.05$. 


\section{CHAPTER IV}

\section{RESULTS}

\section{Exploratory Analysis}

Prior to the analysis of correlations between Fear of Intimacy (FOI), Body Image Avoidance (BIA) and Body Mass Index (BMI), descriptive statistics, frequency distributions and normality tests were examined to determine if the data met assumptions required for correlation analysis. The descriptive statistics revealed normal ranges for the variables of BMI ("Statistics", 2006) and for FOI (Descutner \& Thelen, 1991), as well as revealing a mean age of 21 years in the sample (Table 1). The gender groups consisted of 58 female respondents and 55 male respondents, with $95 \%$ of respondents between 18 and 28 years of age.

Table 1. Descriptive Statistics

\begin{tabular}{|l|c|c|c|c|c|c|c|}
\hline \multicolumn{1}{|c|}{ Variable } & Mean & Std Dev & Skewness & Maximum & Minimum & Median & Kurtosis \\
\hline Age & 21.6194 & 3.5864 & 1.2459 & 33.000 & 18.0000 & 21.0000 & 1.4061 \\
Fear of Intimacy (FOI) & 99.0973 & 12.4706 & -0.1648 & 138.000 & 60.0000 & 99.0000 & 0.7974 \\
Body Mass Index (BMI) & 25.6079 & 4.5855 & 0.5271 & 38.6000 & 14.9000 & 24.8000 & 0.0057 \\
Body Image Avoidance (BIA) & 25.5132 & 9.8706 & 1.2769 & 66.0000 & 8.0000 & 23.0000 & 2.6166 \\
Body Image Avoidance, ln (BIA_log) & 23.7945 & 1.4603 & -0.2286 & 65.9999 & 7.9998 & 22.9999 & 22.9999 \\
Body Image Avoidance, log (BIA_10log) & 23.7945 & 1.46036 & -0.2286 & 65.9998 & 8.0000 & 23.0000 & 0.9754 \\
Body Image Avoidance, square root & 25.1629 & 0.8581 & 0.5665 & 66.5000 & 8.4999 & 23.5000 & 1.0982 \\
(BIA_sqrt) & & & & & & & \\
\hline
\end{tabular}

Preliminary exploratory analysis examined descriptive statistics (Table 1), histograms with normal curves and quantile-quantile plots to visually assess the aforementioned data distributions. A mean score of 25 for BIA (Table 1) was suggestive of further exploration. Increasingly, the extremes to which people experience body image 
disturbance are important and traditionally accepted outliers may in fact reflect valid and important data in this area. The increasing prevalence - both reported and undiagnosed of Body Dysmorphic Disorder (Rosen et al., 1991) and of a normalized discontent with regard to body weight (Rodin et al., 1985) suggest that if an assumption of normality for a score related to body image fails, the scores should be transformed. As a result, values for descriptive statistics for BIA were transformed for natural logarithm function, logarithm in base 10 and square root in an attempt to improve the shape of the distribution (see Table $1 \&$ Table 2). The Shapiro-Wilk W statistic was also used to test data for normality assumptions, due to a sample size below 2000 observations. The results for normality tests are reported in Table 2.

Table 2. Test for Normality

\begin{tabular}{|l|c|c|c|}
\hline \multicolumn{1}{|c|}{ Test for Normality (Shapiro-Wilk test) } \\
\hline \multicolumn{1}{|c|}{ Variable } & Statistic & \multicolumn{2}{c|}{ p Value } \\
\hline Body Image Avoidance (BIA) & 0.9128 & Pr < W & 0.0001 \\
\hline Body Image Avoidance, natural log (BIA_log) & 0.9730 & $\operatorname{Pr}<$ W & 0.0218 \\
\hline Body Image Avoidance, square root (BIA_sqrt) & 0.9647 & $\operatorname{Pr}<\mathrm{W}$ & 0.0045 \\
\hline Body Image Avoidance, $\log$ in base 10 (BIA_log10) & 0.97300 & $\operatorname{Pr}<\mathrm{W}$ & 0.0218 \\
\hline Body Mass Index (BMI) & 0.9757 & $\operatorname{Pr}<\mathrm{W}$ & 0.0376 \\
\hline Fear of Intimacy (FOI) & 0.9893 & $\operatorname{Pr}<\mathrm{W}$ & 0.5205 \\
\hline
\end{tabular}


Of the three aforementioned variables, Body Image Avoidance (BIA)

demonstrated a moderate positive skew (Graph 1.1 and Graph 1.2) and failed to accept the assumption of normality (Table 2). The $P$-value of Shapiro-Wilk's statistic (Table 2) for BIA was 0.001 which provided sufficient evidence to reject the assumption of normality. Histogram and examination of data indicated that there were ten observations that appeared to be outliers which influenced the shape of distribution for BIA (Graph 1.1 and Table 1) however, eight of these outliers did not appear to be true outliers (as defined by more than three inter-quantile ranges away from median). Two observations with extreme values for BIA of 55 and 66 were examined, verified for data errors and determined to be valid for analysis as observations that could potentially reveal valuable information.

Graph 1.1.

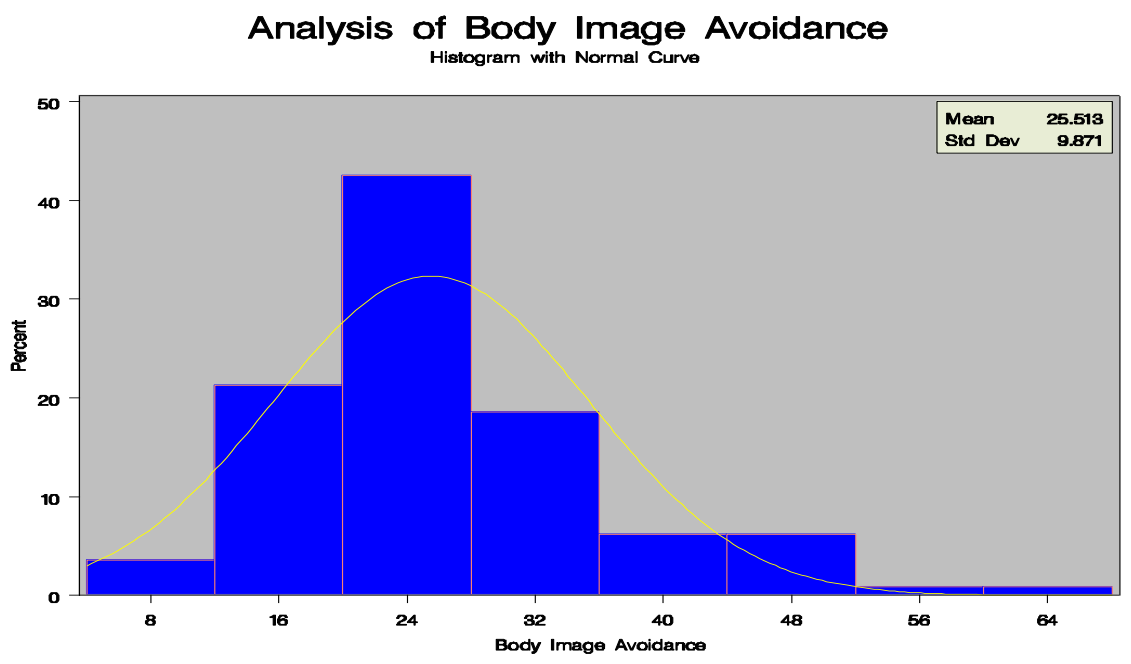


Graph 1.2.
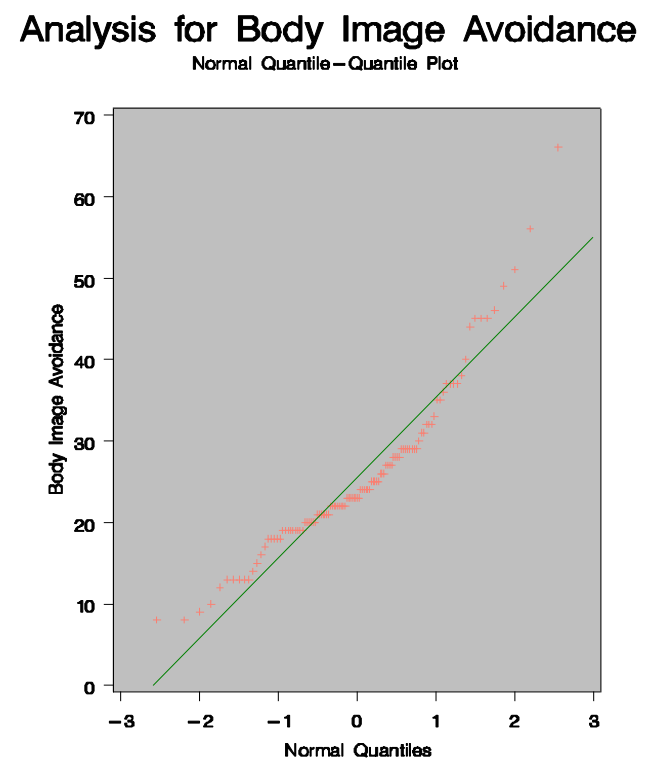

The data transformations were applied to BIA in an attempt to improve the shape of the distribution. Results were reported for natural logarithm function, logarithm in base 10 and square root. Although these transformations did not significantly improve the actual values for the normality test (Table 2), they seem to improve the shape and skew of the distribution (Graph 2.1, 2.2. and 2.3 and Table 1.). Descriptive statistics and histograms for transformed BIA indicate only slight negative skew. Out of the three transformations employed, the natural logarithm transformation was selected as a transformation that better approximates normality than the original distribution and has the lowest skewness coefficient $(-0.22)$ as opposed to 1.27 for non-transformed BIA. For further analysis, the results are reported for original BIA and log transformed BIA (eight of the presented outliers did not appear to be true outliers - as defined by more than three inter-quantile ranges away from median - and observations with extreme values for BIA were examined, verified for data errors and determined to be valid for analysis). 
Graph 1.3.

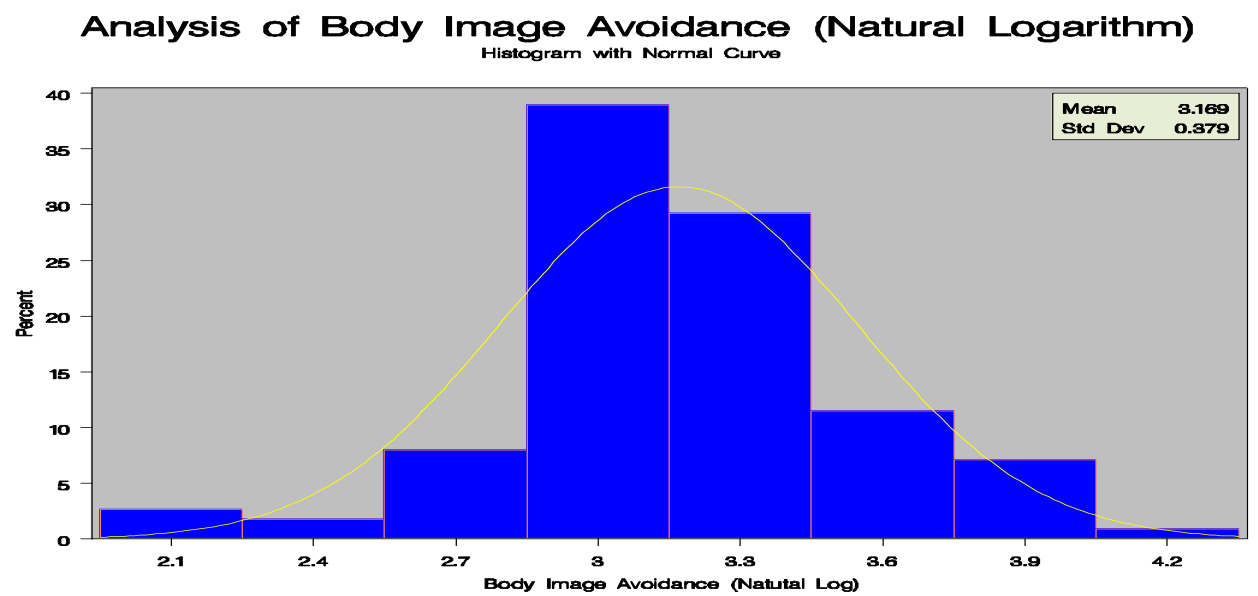

Graph 1.4.

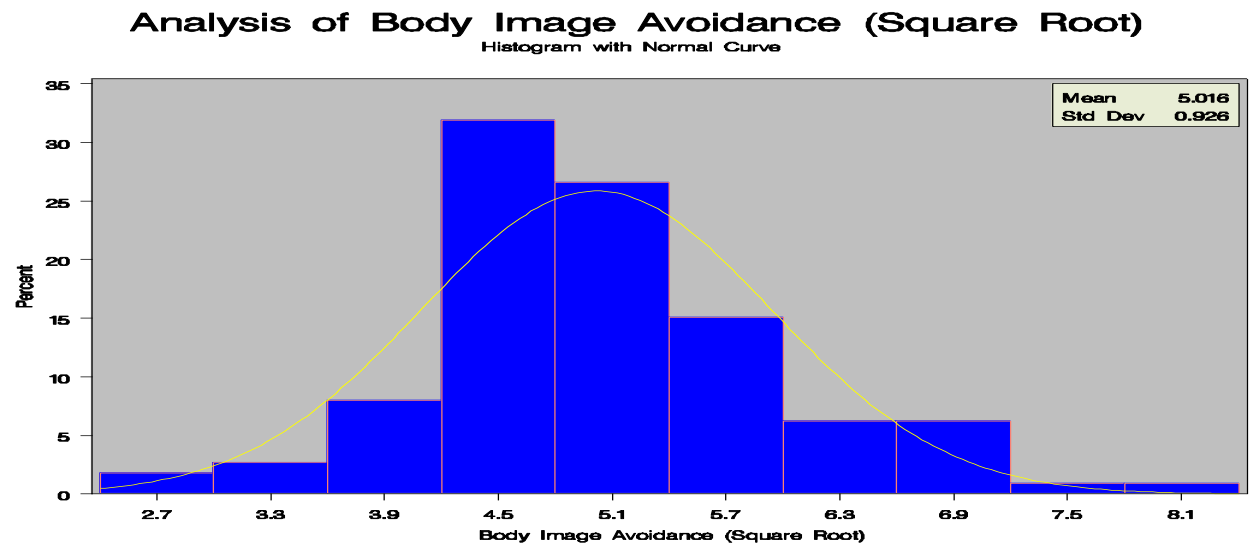

Graph 1.5.

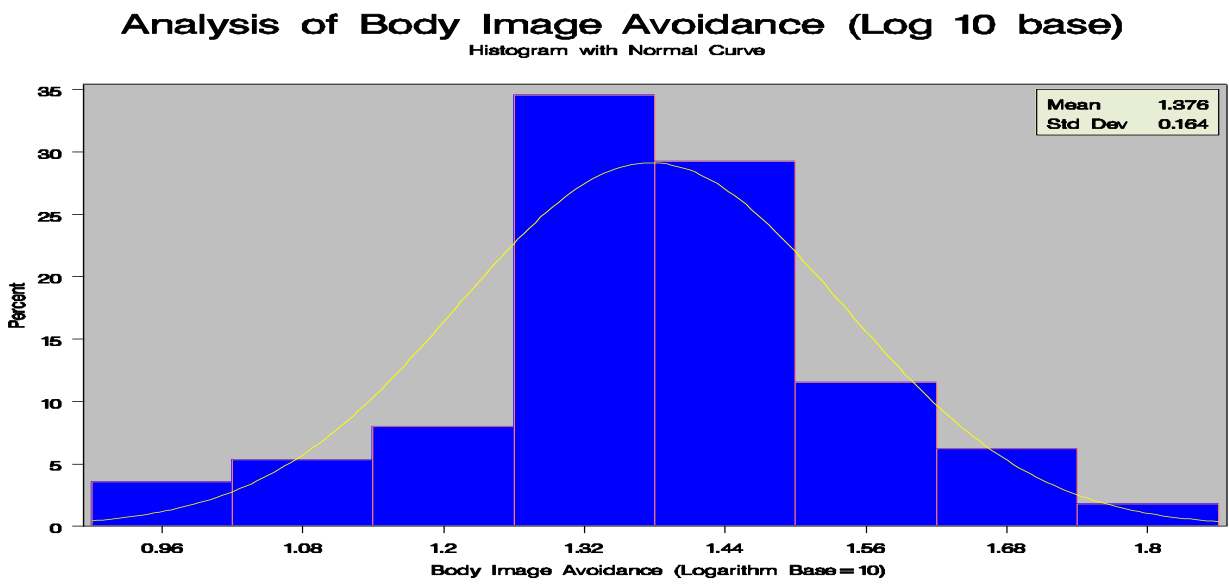


The other variables - Fear of Intimacy (FOI) and Body Mass Index (BMI) in the data set appear to have more normal and symmetrical distributions based upon the descriptive statistics and visual examination of histograms (Graph 3.1, 3.2, 4.1, 4.2 and Table 1). No significant skew was associated with these aforementioned variables. The $P$-value of Shapiro-Wilk's statistic (Table 2) for FOI was 0.52 which provided insufficient evidence to reject the assumption of normality.

Although the Shapiro-Wilk's test (Table 2) did not reject the assumption of normality for BMI, additional visual examination of the frequency distribution noted a large observation of a BMI score of 39 which was examined and validated to be free of data errors. No transformations were applied to these variables.

Graph 2.1.

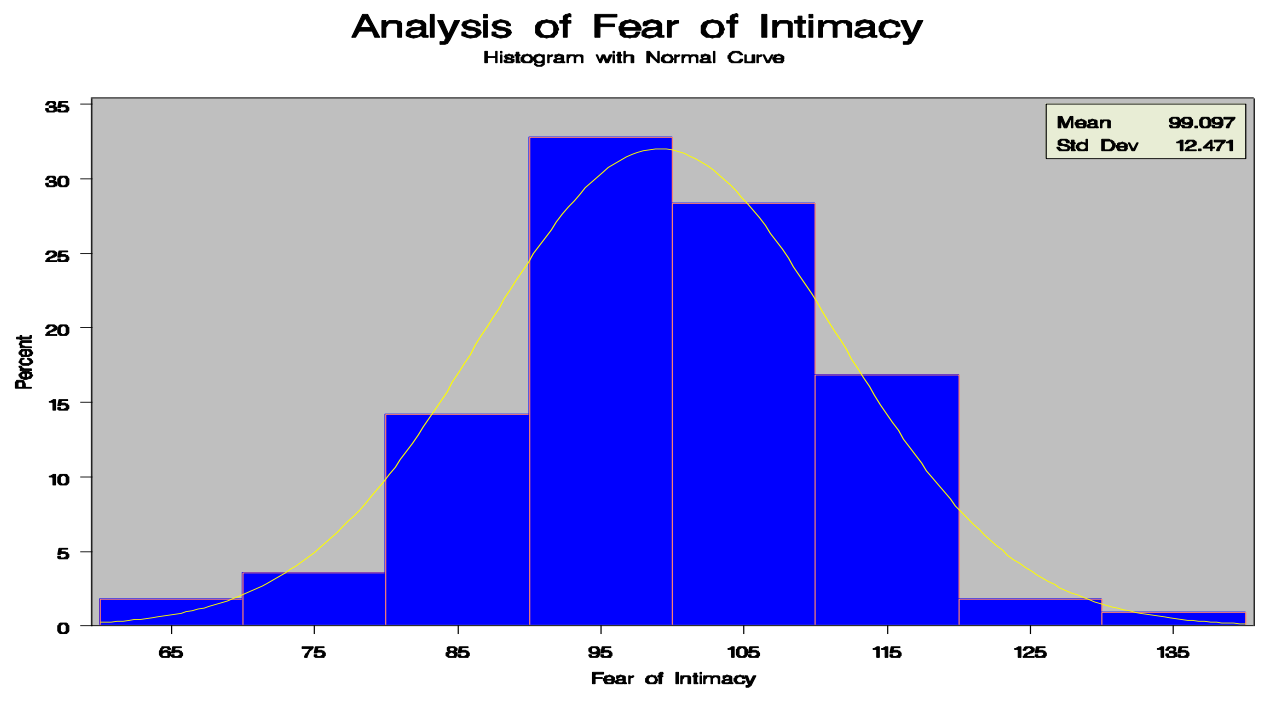


Graph 2.2.

\section{Analysis for Fear of Intimacy}

Normal Quantile-Quantile Plot

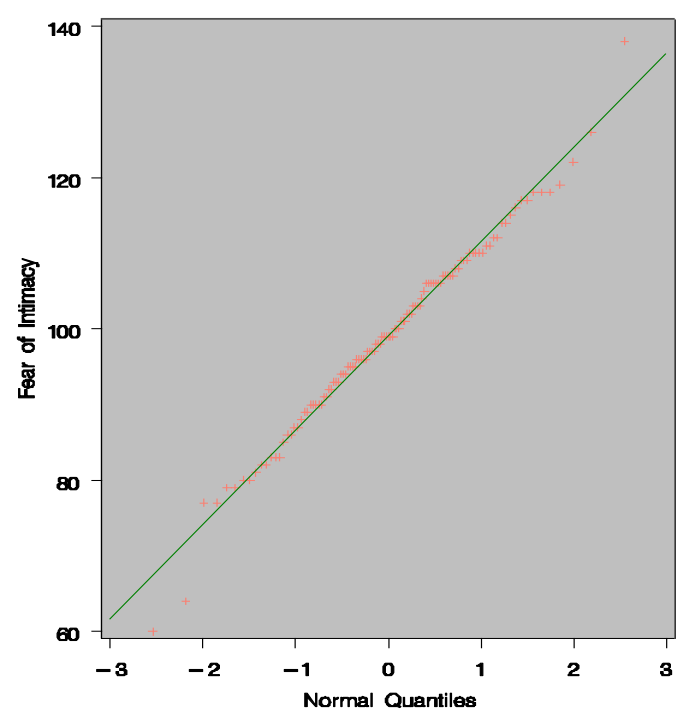

Graph 3.1.

Analysis of Body Mass Index

Histogram with Normal Curve

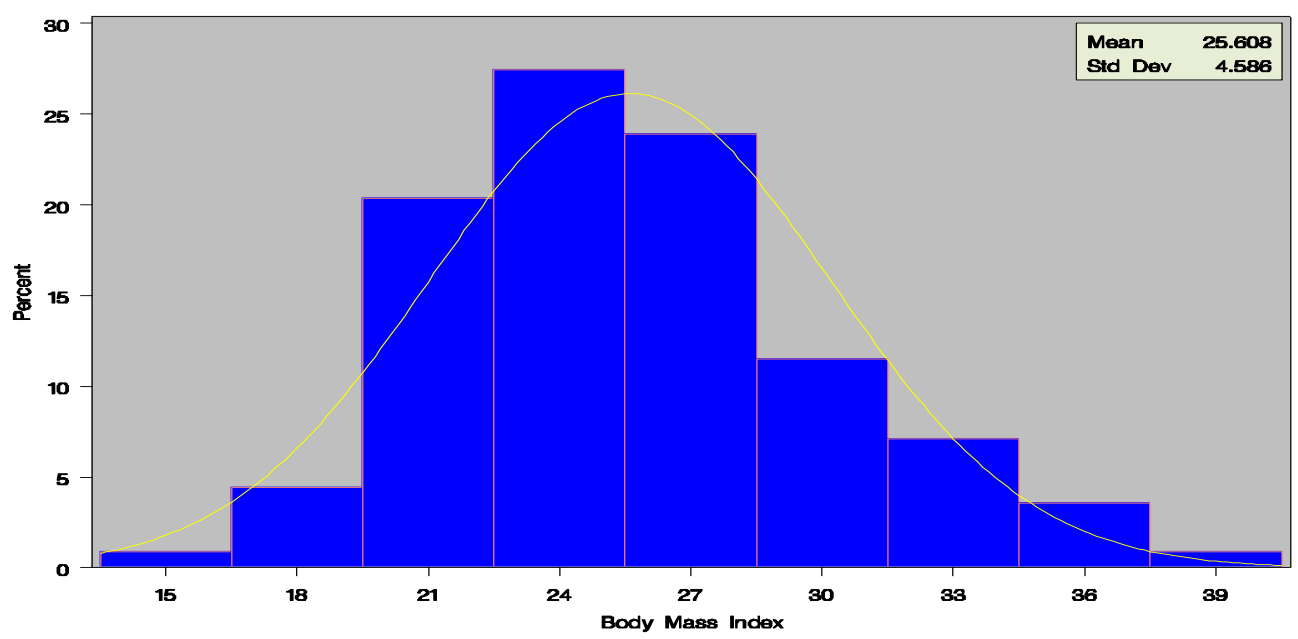


Graph 3.2.
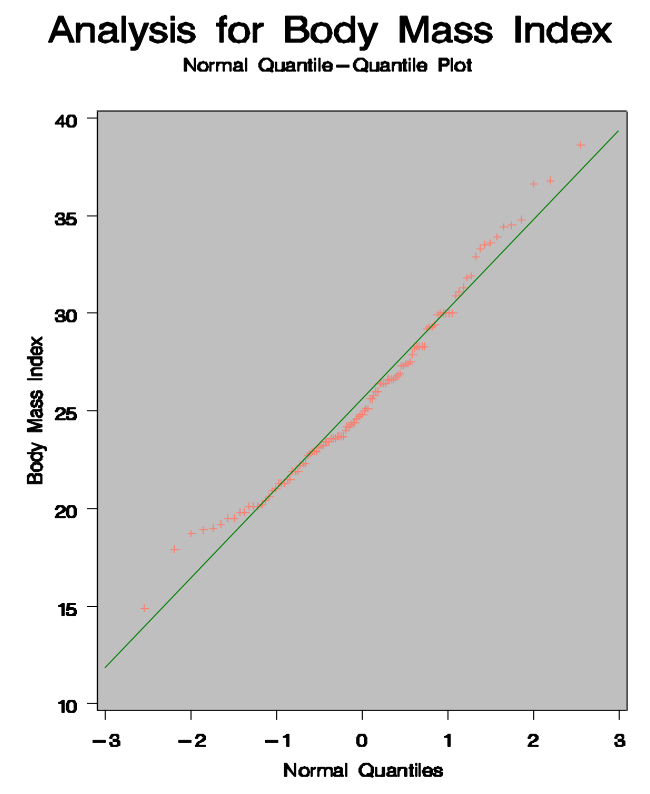

Scatter plots with confidence ellipses (Graph 5.1) were used to test for bivariate normal distributions and to serve as possible indicators of a correlation between two variables. Under bivariate normality, the percentage of observations falling within the confidence ellipse should closely align with the specified confidence level. Confidence ellipses for FOI and BIA, BIA and BMI and BMI and FIO contain the number of observations appropriate to the $95 \%$ confidence level specified.

Confidence ellipses were also examined as visual indicators of correlations; the confidence ellipse recedes diagonally as the correlation between two variables approaches 1 or -1 . The confidence ellipse appears more circular in direction when two variables are uncorrelated. This circular appearance of the confidence ellipse in the plotting of the relationship between BIA and FOI reflects the small correlation that could 
be observed in the correlational matrix. The ellipse in the plotting of the relationship between $\mathrm{BMI}$ and FOI and the relationship between BIA and BMI is somewhat more elongated, reflecting a higher correlation.

Graph 4.1.

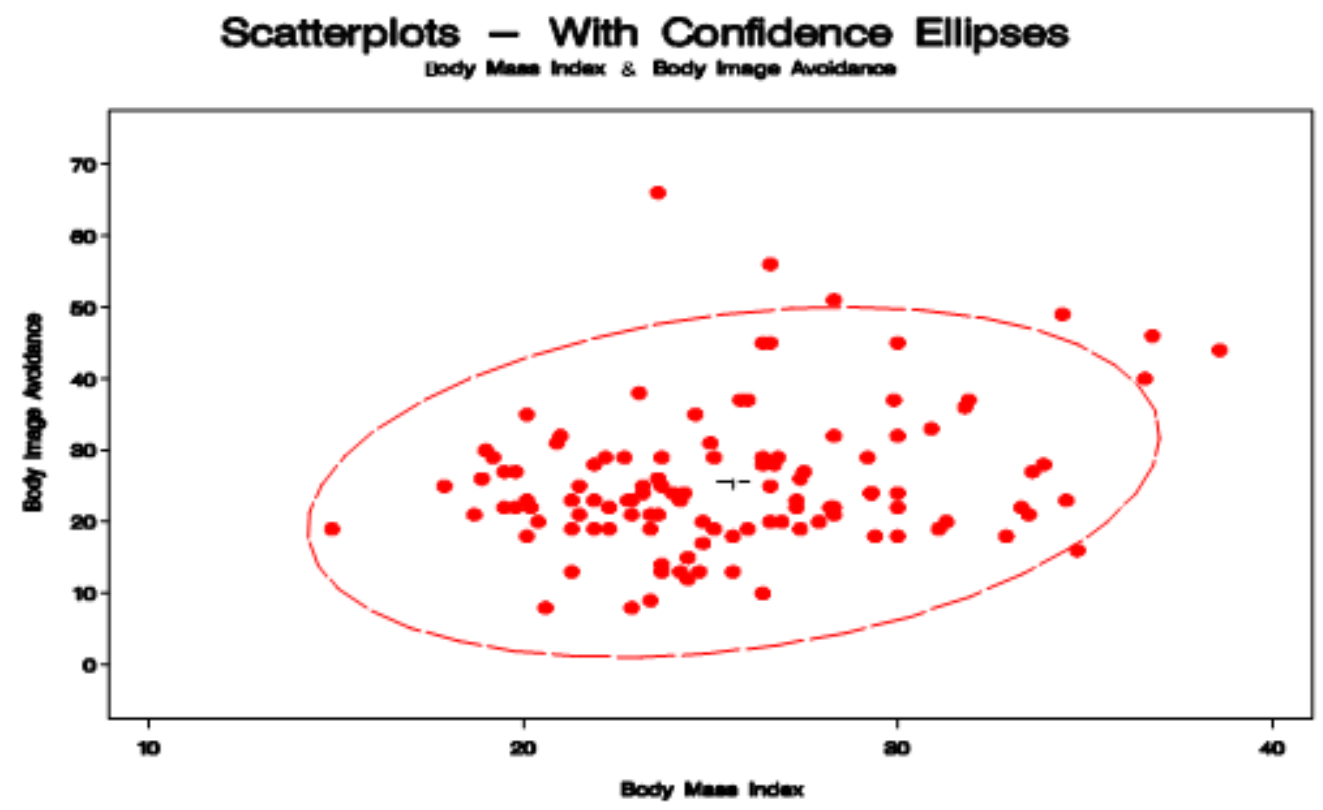


Graph 4.2.

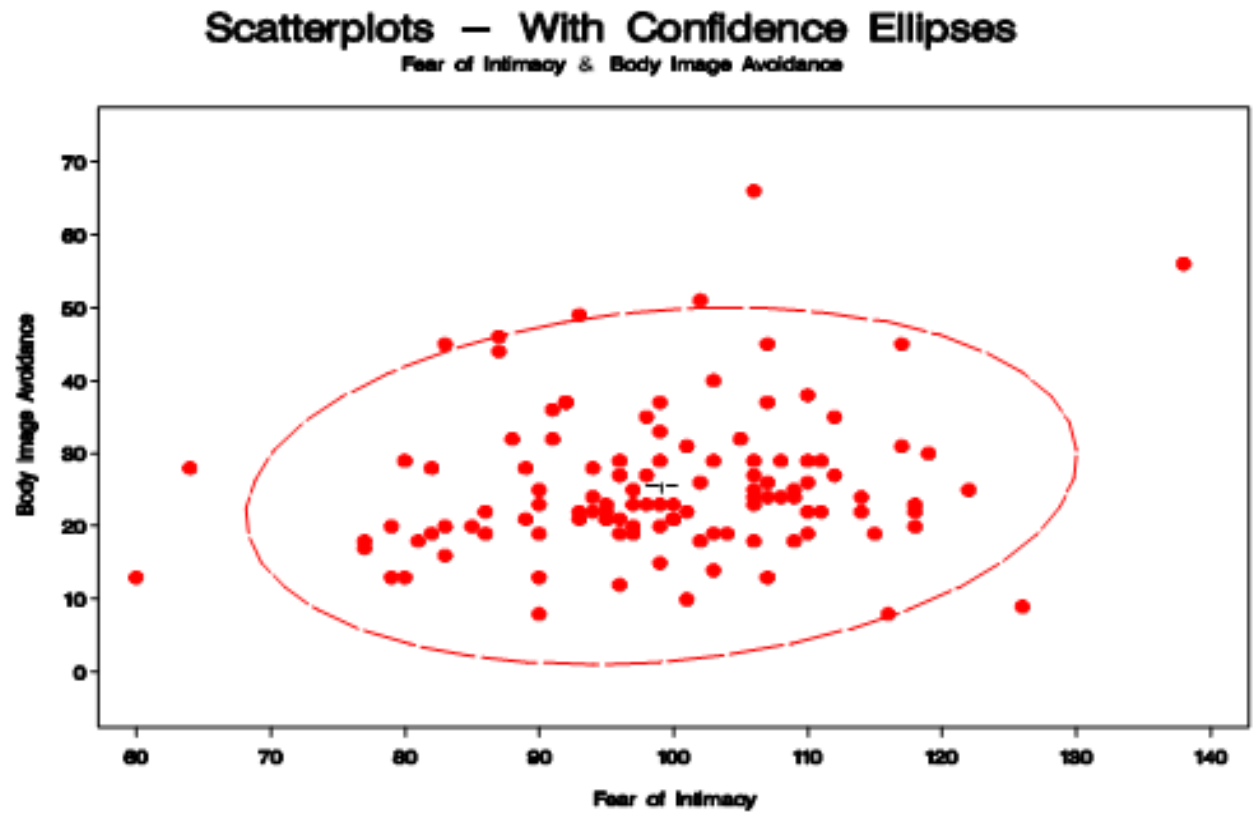

Graph 4.3.

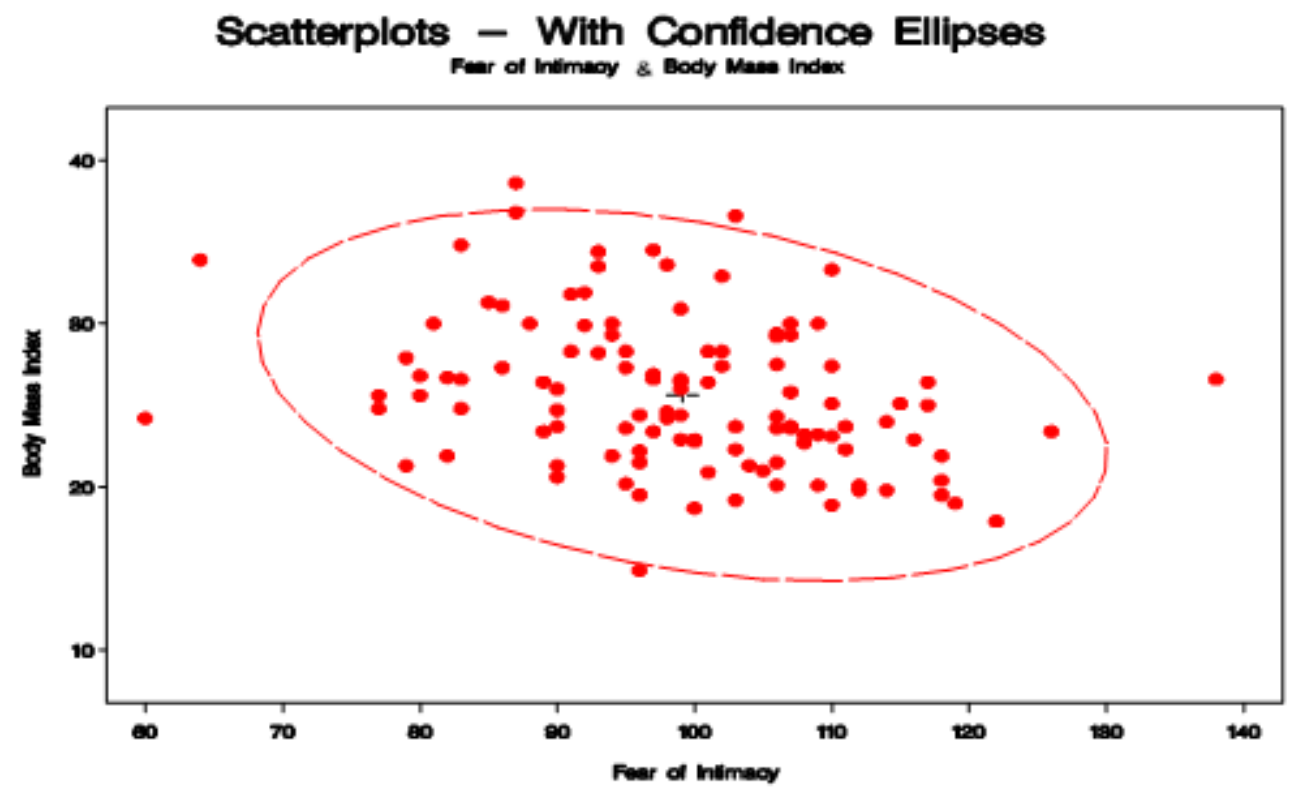


Following the exploratory examination of the raw data, Pearson correlation coefficients were computed for the relationship between BMI and BIA, the relationship between BMI and the relationships between FOI, BIA and FOI (Table 3). The negative correlation is reported in the relationship of BMI and FOI $(-0.32)$ that is significant at $r$ $=.30$. This confirms prior conclusions from scatter plots with confidence ellipses. There is also a significant moderate positive correlation between BMI and BIA (0.2677).

Although the results indicate a weak positive correlation between BIA and FOI, the correlation is not significant based upon a desired medium effect size $(r=.30)$. The correlation coefficient is also not significant for FOI and the natural logarithm transformation for BIA.

Table 3. Matrix of Correlations Between Major Variables.

\begin{tabular}{|l|c|c|c|c|}
\hline \multicolumn{1}{|c|}{ Variable } & $\begin{array}{c}\text { Body Image } \\
\text { Avoidance } \\
\text { (BIA) }\end{array}$ & $\begin{array}{c}\text { Body Image } \\
\text { Avoidance, } \\
\text { natural log } \\
\text { (BIA_log) }\end{array}$ & $\begin{array}{c}\text { Body Mass } \\
\text { Index (BMI) }\end{array}$ & $\begin{array}{c}\text { Fear of } \\
\text { Intimacy } \\
\text { (FOI) }\end{array}$ \\
\hline \multicolumn{1}{|c|}{ With Variable } & & & & \\
Avody Image & 1 & $\begin{array}{l}0.95566 \\
(<.0001)\end{array}$ & $\begin{array}{c}0.26777 \\
(<0.0041)\end{array}$ & $\begin{array}{c}0.16918 \\
(<0.0732)\end{array}$ \\
\hline $\begin{array}{l}\text { Body Image } \\
\text { Avoidance, natural log } \\
\text { (BIA_log) }\end{array}$ & $\begin{array}{l}0.95566 \\
(<.0001)\end{array}$ & 1 & $\begin{array}{c}0.25141 \\
(<0.0072)\end{array}$ & $\begin{array}{c}0.14885 \\
(<0.1156)\end{array}$ \\
\hline $\begin{array}{l}\text { Body Mass Index } \\
\text { (BMI) }\end{array}$ & $\begin{array}{c}0.26777 \\
(<0.0041)\end{array}$ & $\begin{array}{c}0.25141 \\
(<0.0072)\end{array}$ & $\begin{array}{l}-0.32612 \\
(<0.0004)\end{array}$ \\
\hline $\begin{array}{l}\text { Fear of Intimacy } \\
\text { (FOI) }\end{array}$ & $\begin{array}{l}0.16918 \\
(<0.0732)\end{array}$ & $\begin{array}{l}0.14885 \\
(<0.1156)\end{array}$ & $\begin{array}{c}-0.32612 \\
(<0.0004)\end{array}$ & 1 \\
\hline
\end{tabular}


Table 4. Matrix of Correlations by Gender.

\begin{tabular}{|c|c|c|c|c|c|c|c|c|}
\hline \multirow{2}{*}{$\begin{array}{l}\text { Variable } \\
\text { With }\end{array}$} & \multicolumn{2}{|c|}{$\begin{array}{c}\text { Body Image } \\
\text { Avoidance (BIA) }\end{array}$} & \multicolumn{2}{|c|}{$\begin{array}{c}\text { Body Image } \\
\text { Avoidance, } \\
\text { natural log } \\
\text { (BIA_log) }\end{array}$} & \multicolumn{2}{|c|}{$\begin{array}{l}\text { Body Mass Index } \\
\text { (BMI) }\end{array}$} & \multicolumn{2}{|c|}{$\begin{array}{c}\text { Fear of Intimacy } \\
\text { (FOI) }\end{array}$} \\
\hline & & & & & & & & \\
\hline Gender & Female & Male & Female & Male & Female & Male & Female & Male \\
\hline $\begin{array}{l}\text { Body } \\
\text { Image } \\
\text { Avoidance } \\
\text { (BIA) }\end{array}$ & 1 & 1 & $\begin{array}{l}0.95566 \\
(<.0001)\end{array}$ & $\begin{array}{l}0.95566 \\
(<.0001)\end{array}$ & $\begin{array}{r}0.25610 \\
(<0.0521)\end{array}$ & $\begin{array}{l}0.28642 \\
(<0.0336)\end{array}$ & $\begin{array}{c}0.2362 \\
(<0.0741)\end{array}$ & $\begin{array}{l}0.10697 \\
(<0.4387)\end{array}$ \\
\hline $\begin{array}{l}\text { Body } \\
\text { Image } \\
\text { Avoidance, } \\
\text { natural log } \\
\text { (BIA_log) }\end{array}$ & $\begin{array}{l}0.95566 \\
(<.0001)\end{array}$ & $\begin{array}{l}0.95566 \\
(<.0001)\end{array}$ & 1 & 1 & $\begin{array}{r}0.23461 \\
(<0.0762)\end{array}$ & $\begin{array}{l}0.27856 \\
(<0.0391)\end{array}$ & $\begin{array}{c}0.2117 \\
(<0.0762)\end{array}$ & $\begin{array}{l}0.09239 \\
(<0.5040)\end{array}$ \\
\hline $\begin{array}{l}\text { Body Mass } \\
\text { Index } \\
\text { (BMI) }\end{array}$ & $\begin{array}{l}0.25610 \\
(<0.0521)\end{array}$ & $\begin{array}{l}0.28642 \\
(<0.0336)\end{array}$ & $\begin{array}{l}0.23461 \\
(<0.0762)\end{array}$ & $\begin{array}{l}0.27856 \\
(<0.0391)\end{array}$ & 1 & 1 & $\begin{array}{l}-0.3404 \\
(<0.0085)\end{array}$ & $\begin{array}{l}-0.3510 \\
(<0.0082)\end{array}$ \\
\hline $\begin{array}{l}\text { Fear of } \\
\text { Intimacy } \\
\text { (FOI) }\end{array}$ & $\begin{array}{c}0.2362 \\
(<0.0741)\end{array}$ & $\begin{array}{l}0.10697 \\
(<0.4387)\end{array}$ & $\begin{array}{c}0.2117 \\
(<0.0762)\end{array}$ & $\begin{array}{l}0.09239 \\
(<0.5040)\end{array}$ & $\begin{array}{l}-0.3404 \\
(<0.0085)\end{array}$ & $\begin{array}{l}-0.3510 \\
(<0.0082)\end{array}$ & 1 & 1 \\
\hline
\end{tabular}

Next, the sample population was tested to determine differences between gender groups; the correlation coefficients from gender groups were calculated (see Table 4) and tested for equality using Fishers' R-to-Z transformation (see Table 5). The $p$-value for testing $H_{0}$ is derived by treating the difference $z_{1}-z_{2}$ as a normal random variable with a mean of zero and variance of $1 /\left(n_{1}-3\right)+1 /\left(n_{2}-3\right)$, where $z_{1}$ and $z_{2}$ are Fisher's $z$ transformation of the sample correlations $r_{1}$ and $r_{2}$, respectively, and where $n_{1}$ and $n_{2}$ are the corresponding sample sizes. 
Table 5. Test of Equality of Population Correlations by Gender (Fisher R-to-Z Transformation Test)

\begin{tabular}{|l|l|c|c|c|c|c|c|c|}
\hline \multicolumn{1}{|l|}{ Variable } & $\begin{array}{c}\text { With } \\
\text { Variable }\end{array}$ & $\begin{array}{c}\text { sample } \\
\text { size } \\
\text { (Female) }\end{array}$ & $\begin{array}{c}\text { Fisher's } \\
\mathbf{Z} \\
\text { (Female) }\end{array}$ & $\begin{array}{c}\text { sample } \\
\text { size } \\
\text { (Male) }\end{array}$ & $\begin{array}{c}\text { Fisher's } \\
\mathbf{Z} \\
\text { (Male) }\end{array}$ & variance & $\mathbf{Z}$ & Pval \\
\hline $\begin{array}{l}\text { Fear of } \\
\text { Intimacy } \\
\text { (FOI) }\end{array}$ & $\begin{array}{l}\text { Body } \\
\text { Image } \\
\text { Avoidance } \\
\text { (BIA) }\end{array}$ & 58 & 0.2408 & 55 & 0.1073 & 0.0374 & 0.6897 & 0.4903 \\
\hline $\begin{array}{l}\text { Fear of } \\
\text { Intimacy } \\
\text { (FOI) }\end{array}$ & $\begin{array}{l}\text { Body Mass } \\
\text { Index } \\
\text { (BMI) }\end{array}$ & 58 & -0.3546 & 55 & -0.3666 & 0.0374 & 0.0623 & 0.9502 \\
\hline $\begin{array}{l}\text { Fear of } \\
\text { Intimacy } \\
\text { (FOI) }\end{array}$ & $\begin{array}{l}\text { Body } \\
\text { Image } \\
\text { Avoidance, } \\
\text { natural log } \\
\text { (BIA_log) }\end{array}$ & 58 & 0.21504 & 55 & 0.09266 & 0.037413 & 0.63273 & 0.52691 \\
\hline $\begin{array}{l}\text { Body } \\
\text { Mass } \\
\text { Index } \\
\text { (BMI) }\end{array}$ & $\begin{array}{l}\text { Body } \\
\text { Image } \\
\text { Avoidance, } \\
\text { natural log } \\
\text { (BIA_log) }\end{array}$ & 58 & 0.23906 & 55 & 0.28612 & 0.037413 & -0.2433 & 0.80776 \\
\hline $\begin{array}{l}\text { Body } \\
\text { Image } \\
\text { Avoidance } \\
\text { (BIA) }\end{array}$ & $\begin{array}{l}\text { Body Mass } \\
\text { Index } \\
\text { (BMI) }\end{array}$ & 58 & 0.2619 & 55 & 0.2946 & 0.0374 & -0.1692 & 0.8656 \\
\hline
\end{tabular}

Fisher's R-to-Z transformations were conducted to test the difference between BIA and BMI, BIA and FOI and FOI and BIA for male and female samples (see Table 5). The $p$-values that range from 0.49 to 0.95 do not provide evidence to reject the null hypothesis that the correlations between scores are different for male and female. Despite prospective power analyses that were conducted to estimate sample size, the sample sizes $n_{1}=58$ (female) and $n_{2}=55$ (male) were large enough to detect correlations of size .0 .3 or more, however, the observed correlations were less than this. This is also evident from the examining scatter plots of scores by gender. 
Despite the initial treatment of only FOI, BIA, BMI and gender as variables, exploratory analyses revealed that differences in scores based upon age were also worthy of further investigation. The histogram below shows the distribution of respondents by age.

Graph 5.1. Distribution of Age

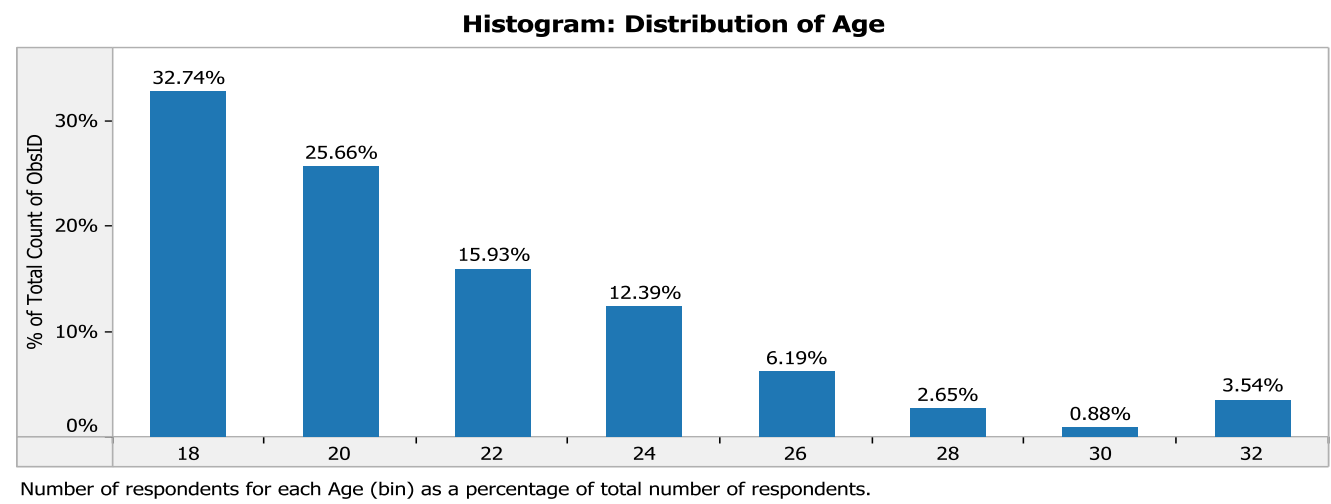

Since the sample population was comprised of students on a college campus, 95 $\%$ of respondents were aged 28 or less. Prior to analysis, the additional variable of age was binned into two equal categories: group 1 (less than or equal to age 22) and group 2 (older than age 22). Correlation coefficients for FIO, BMI and BIA were computed for two age groups (group 1 and group 2) (see Table 6) and Fisher's R-to-Z transformation was used to test the difference between correlation coefficients between scores for two samples (see Table 7). 
Table 6. Matrix of Correlations by Age

\begin{tabular}{|c|c|c|c|c|c|c|c|c|}
\hline \multirow{2}{*}{$\begin{array}{l}\text { Variable } \\
\text { With }\end{array}$} & \multicolumn{2}{|c|}{$\begin{array}{c}\text { Body Image } \\
\text { Avoidance (BIA) }\end{array}$} & \multicolumn{2}{|c|}{$\begin{array}{c}\text { Body Image } \\
\text { Avoidance, } \\
\text { natural log } \\
\text { (BIA_log) }\end{array}$} & \multicolumn{2}{|c|}{$\begin{array}{c}\text { Body Mass Index } \\
\text { (BMI) }\end{array}$} & \multicolumn{2}{|c|}{$\begin{array}{c}\text { Fear of Intimacy } \\
\text { (FOI) }\end{array}$} \\
\hline & & & & & & & & \\
\hline Age & $\begin{array}{c}\text { Group } \\
1\end{array}$ & $\begin{array}{c}\text { Group } \\
2\end{array}$ & $\begin{array}{c}\text { Group } \\
1\end{array}$ & $\begin{array}{c}\text { Group } \\
2\end{array}$ & $\begin{array}{c}\text { Group } \\
1\end{array}$ & $\begin{array}{c}\text { Group } \\
2\end{array}$ & $\begin{array}{c}\text { Group } \\
1\end{array}$ & $\begin{array}{c}\text { Group } \\
2\end{array}$ \\
\hline $\begin{array}{l}\text { Body Image } \\
\text { Avoidance (BIA) }\end{array}$ & 1 & 1 & - & - & $\begin{array}{c}0.3349 \\
(0.0057)\end{array}$ & $\begin{array}{r}0.1845 \\
(0.2157)\end{array}$ & $\begin{array}{l}-0.0148 \\
(0.9063)\end{array}$ & $\begin{array}{r}0.4139 \\
(0.0035)\end{array}$ \\
\hline $\begin{array}{l}\text { Body Image } \\
\text { Avoidance, } \\
\text { natural log } \\
\text { (BIA_log) } \\
\end{array}$ & - & - & 1 & 1 & $\begin{array}{l}0.26573 \\
(0.0307)\end{array}$ & $\begin{array}{l}0.21799 \\
(0.1417)\end{array}$ & $\begin{array}{l}-0.0181 \\
(0.8853)\end{array}$ & $\begin{array}{r}0.4127 \\
(0.0036)\end{array}$ \\
\hline $\begin{array}{l}\text { Body Mass Index } \\
\text { (BMI) }\end{array}$ & $\begin{array}{l}0.3349 \\
(0.0057)\end{array}$ & $\begin{array}{r}0.1845 \\
(0.2157)\end{array}$ & $\begin{array}{l}0.2657 \\
(0.0307)\end{array}$ & $\begin{array}{l}0.2179 \\
(0.1417)\end{array}$ & 1 & 1 & $\begin{array}{l}-0.3954 \\
(0.0009)\end{array}$ & $\begin{array}{l}-0.2053 \\
(0.1671)\end{array}$ \\
\hline $\begin{array}{l}\text { Fear of Intimacy } \\
\text { (FOI) }\end{array}$ & $\begin{array}{l}-0.0148 \\
(0.9063)\end{array}$ & $\begin{array}{r}0.4139 \\
(0.0035)\end{array}$ & $\begin{array}{l}-0.0181 \\
(0.8853)\end{array}$ & $\begin{array}{c}0.4127 \\
(0.0036)\end{array}$ & $\begin{array}{l}-0.3954 \\
(0.0009)\end{array}$ & $\begin{array}{l}-0.2053 \\
(0.1671)\end{array}$ & 1 & 1 \\
\hline
\end{tabular}

Table 7. Test of Equality of Population Correlations by Age (Fisher R-to-Z Transformation Test)

\begin{tabular}{|l|l|c|c|c|c|c|c|c|}
\hline Variable & $\begin{array}{c}\text { With } \\
\text { Variable }\end{array}$ & $\begin{array}{c}\text { sample } \\
\text { size } \\
\text { (Female) }\end{array}$ & $\begin{array}{c}\text { Fisher's } \\
\mathbf{Z} \\
\text { (Female) }\end{array}$ & $\begin{array}{c}\text { sample } \\
\text { size } \\
\text { (Male) }\end{array}$ & $\begin{array}{c}\text { Fisher's } \\
\mathbf{Z} \\
\text { (Male) }\end{array}$ & variance & $\mathbf{Z}$ & Pval \\
\hline $\begin{array}{l}\text { Fear of } \\
\text { Intimacy } \\
\text { (FOI) }\end{array}$ & $\begin{array}{l}\text { Body } \\
\text { Image } \\
\text { Avoidance } \\
\text { (BIA) }\end{array}$ & 66 & -0.0148 & 47 & 0.44039 & 0.03860 & -2.3170 & 0.0205 \\
\hline $\begin{array}{l}\text { Fear of } \\
\text { Intimacy } \\
\text { (FOI) }\end{array}$ & $\begin{array}{l}\text { Body Mass } \\
\text { Index } \\
\text { (BMI) }\end{array}$ & 66 & -0.4182 & 47 & -20829 & 0.03860 & -1.0685 & 0.2853 \\
\hline $\begin{array}{l}\text { Fear of } \\
\text { Intimacy } \\
\text { (FOI) }\end{array}$ & $\begin{array}{l}\text { Body } \\
\text { Image } \\
\text { Avoidance, } \\
\text { natural log } \\
\text { (BIA_log) }\end{array}$ & 66 & -0.0181 & 47 & 0.4388 & 0.03860 & -2.3261 & 0.0200 \\
\hline $\begin{array}{l}\text { Body } \\
\text { Mass } \\
\text { Index } \\
\text { (BMI) }\end{array}$ & $\begin{array}{l}\text { Body } \\
\text { Image } \\
\text { Avoidance, } \\
\text { natural log } \\
\text { (BIA_log) }\end{array}$ & 66 & 0.2722 & 47 & 0.2215 & 0.03860 & 0.2582 & 0.7963 \\
\hline $\begin{array}{l}\text { Body } \\
\text { Image } \\
\text { Avoidance } \\
\text { (BIA) }\end{array}$ & $\begin{array}{l}\text { Body Mass } \\
\text { Index } \\
\text { (BMI) }\end{array}$ & 66 & 0.3483 & 47 & 0.18666 & 0.03860 & 0.8232 & 0.4104 \\
\hline
\end{tabular}


Of the correlations tested (BMI and BIA, FOI and BIA, FOI and BMI), the value for the difference of correlation between FOI and BIA that is equal to 0.02 provides evidence that these correlations for age group 1 and age group 2 are significantly different. This is supported from the visual examination of the scatter plot between FOI and BIA (see Graph 7.1) for the population split by those who are less than 22 years old and more than 22. The scatter plot of FOI and BIA for group 1 shows a weak negative relationship, while the scatter plot of FOI and BIA for group 2 reveals a strong positive slope.

There is no difference indicated in the correlations between FOI and BMI and BMI and BIA for two age groups as evident from the visual examinations of scatter plots (See Graph 7.2 and 7.3). $P$-values for the Fisher's statistics $(p=0.2853$ for FOI and BMI and $p=0.4104$ for BIA and BMI) fail to reject the null hypothesis that the correlations between the two scores are different for two age groups (see Table 7).

\section{Hypothesis \#1: As body mass index increases, fear of intimacy increases.}

This hypothesis is supported at $p<0.05$. Correlation coefficient $=-0.3261$, significant at $\mathrm{p}<0.05$. This correlation can also be observed from the scatterplot with confidence ellipses (See Graph 5.1).

\section{Hypothesis \#2: The relationship of body mass index and fear of intimacy varies by gender.}

This hypothesis is not supported. The correlation coefficient between BMI and FOI for the male sample is $-0.3510(p<0.05)$. The correlation coefficient between BMI and FOI 
for the female sample is $-0.3404(p<0.05)$ (see Table 4). Visual examination of the scatterplots of the correlation between FOI and BMI for female and male also does not indicate that these two correlations are significantly different (see Graph 6.1).

Graph 6.1.

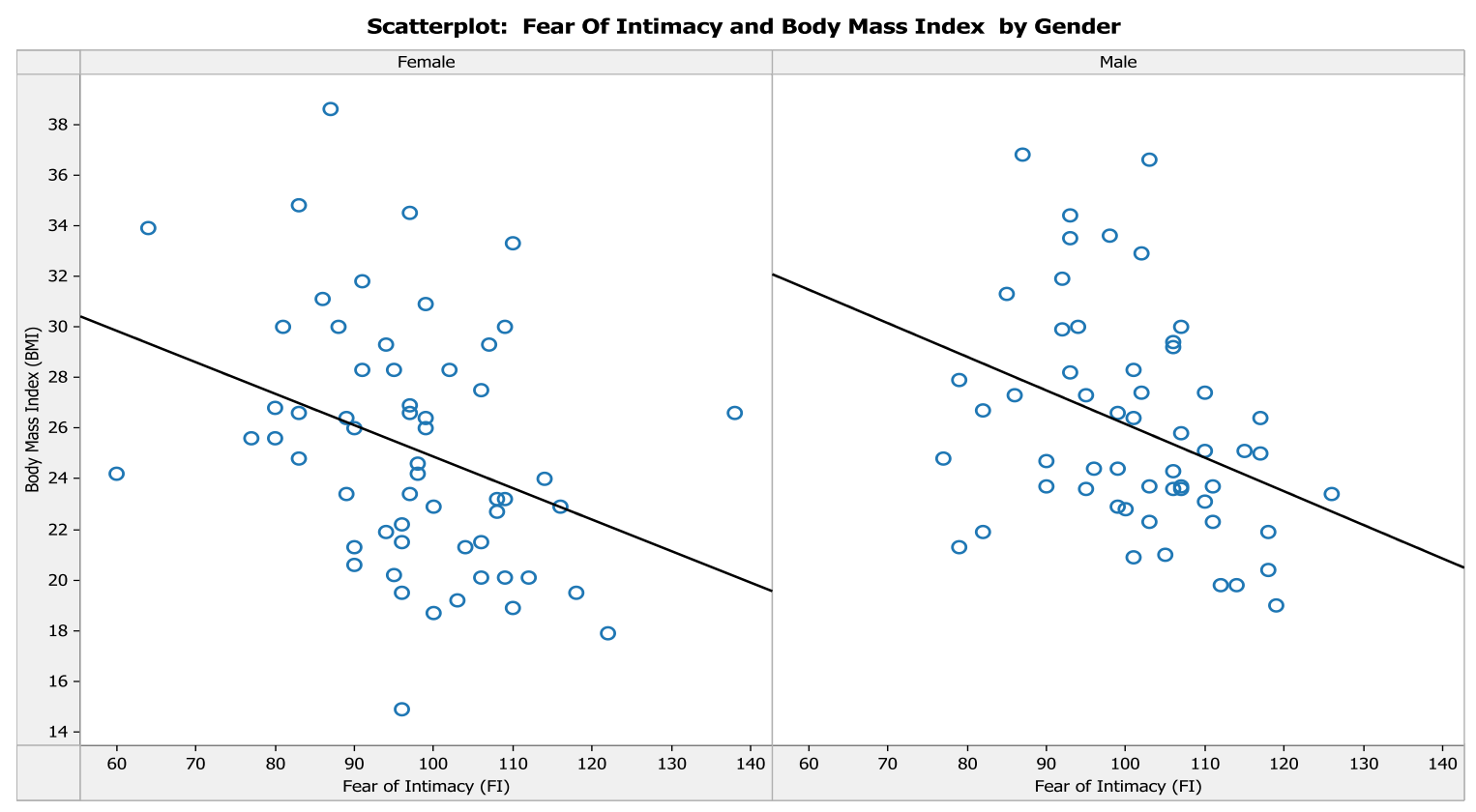

Fear of Intimacy (FI) vs. Body Mass Index (BMI) broken down by Gender(bin).

Fisher's tests for the difference between these two correlations reports $p$-value $=$ 0.9502, which does not provide evidence to reject the null hypothesis that the correlations between scores are equal for male and female. The sample sizes $n 1=58$ (female) and $n 2$ $=55$ (male) could also not be large enough to detect a desired medium effect size of $\mathrm{r}=$. 30 (see Table 5). 


\section{Hypothesis \#3: As body image avoidance increases, fear of intimacy increases.}

This hypothesis is not supported, however, $p$ approached significance, at $p<0.07$ with a correlation coefficient equal to -0.1691 (see Table 3). This correlation can also be observed from the scatterplot with confidence ellipses (See Graph 5.2).

\section{Hypothesis \#4: The relationship of body image avoidance and fear of intimacy varies by gender.}

This hypothesis is not supported; the correlation coefficient between BIA and FOI for male sample is $-0.1070(p<0.43)$. The correlation coefficient between BIA and FOI for female sample is $-0.2362(p<0.07)$ (see Table 4). Visual examination of the correlation between BIA and FOI for female and male also does not indicate that these two correlations are significantly different (see Graph 6.2).

\section{Graph 6.2.}

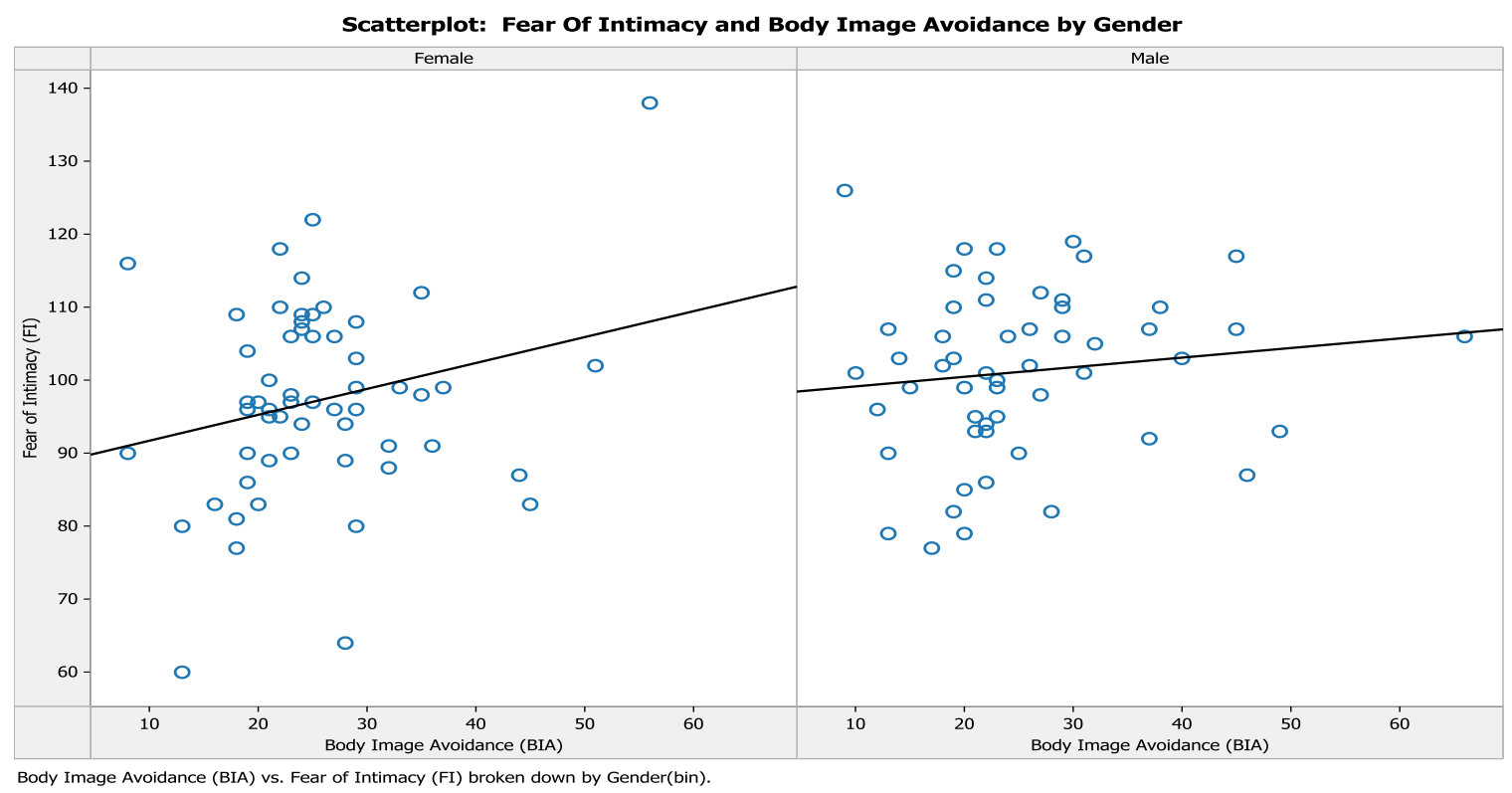


Fisher's R-to-Z transformation revealed that the difference between these two correlations yield a $p$-value $=0.4903$, which does not provide evidence to reject the null hypothesis that the correlations between scores are equal for male and female (see Table $5)$.

Hypothesis \#5: As body mass index increases, body image avoidance increases. This hypothesis is supported at $p<0.05$, with a correlation coefficient $=0.2677$. This correlation can also be observed from the scatterplot with confidence ellipses (See Graph $5.1)$.

Hypothesis \#6: The relationship of body mass index and body image avoidance varies by gender.

This hypothesis is not supported. The correlation coefficient between BIA and BMI for male population is $0.2864(p<0.05)$ and equal to $0.2561(p>0.0521)$ for female sample (see Table 4). Visual examination of the correlation between BIA and BMI for female and male sample also does not show that these two correlations are significantly different (see Graph 6.3). 
Graph 6.3.

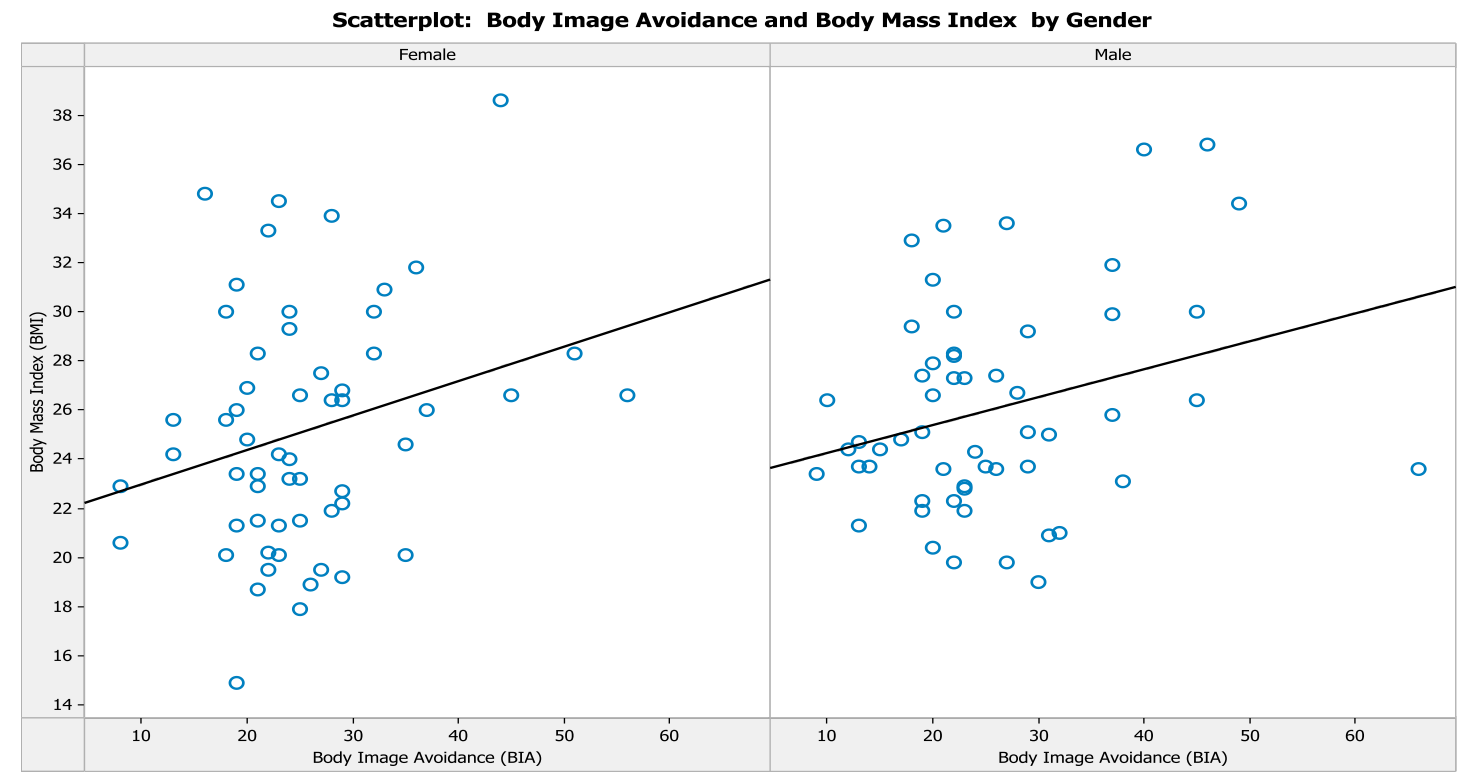

Body Image Avoidance (BIA) vs. Body Mass Index (BMI) broken down by Gender(bin).

Fisher's R-to-Z transformation for the difference between these two correlations reported a $p$-value $=0.8656$ which does not provide evidence to reject the null hypothesis that the correlations between scores are equal for male and female (see Table 5).

The inclusion of age as a variable resulted in the treatment of an additional 3 hypotheses. Using Fisher's R-to-Z transformation, the data was assessed to determine a difference between correlation coefficients between scores for two samples split by age. Prior to analysis, the additional variable of age was binned into two equal categories: group 1 - less than or equal to age 22 , with group 2 - older than 22 . Since the sample population was comprised of students on a college campus, $95 \%$ of respondents were aged 28 or less. 
Hypothesis \#7: The relationship of body mass index and fear of intimacy varies from ages 18 to 22 (group 1) to ages 23 to 34 (group 2).

This hypothesis is not supported; the correlation coefficient between FOI and BMI for Group 1 is $-0.39543(p<0.05)$. Correlation coefficient between FOI and BMI for Group 2 is $-0.20533(p<0.1671)$ (see Table 6). Visual examination of the correlation between FOI and BMI for group 1 and group 2 also does not show that these two correlations are significantly different (see Graph 7.1).

\section{Graph 7.1.}

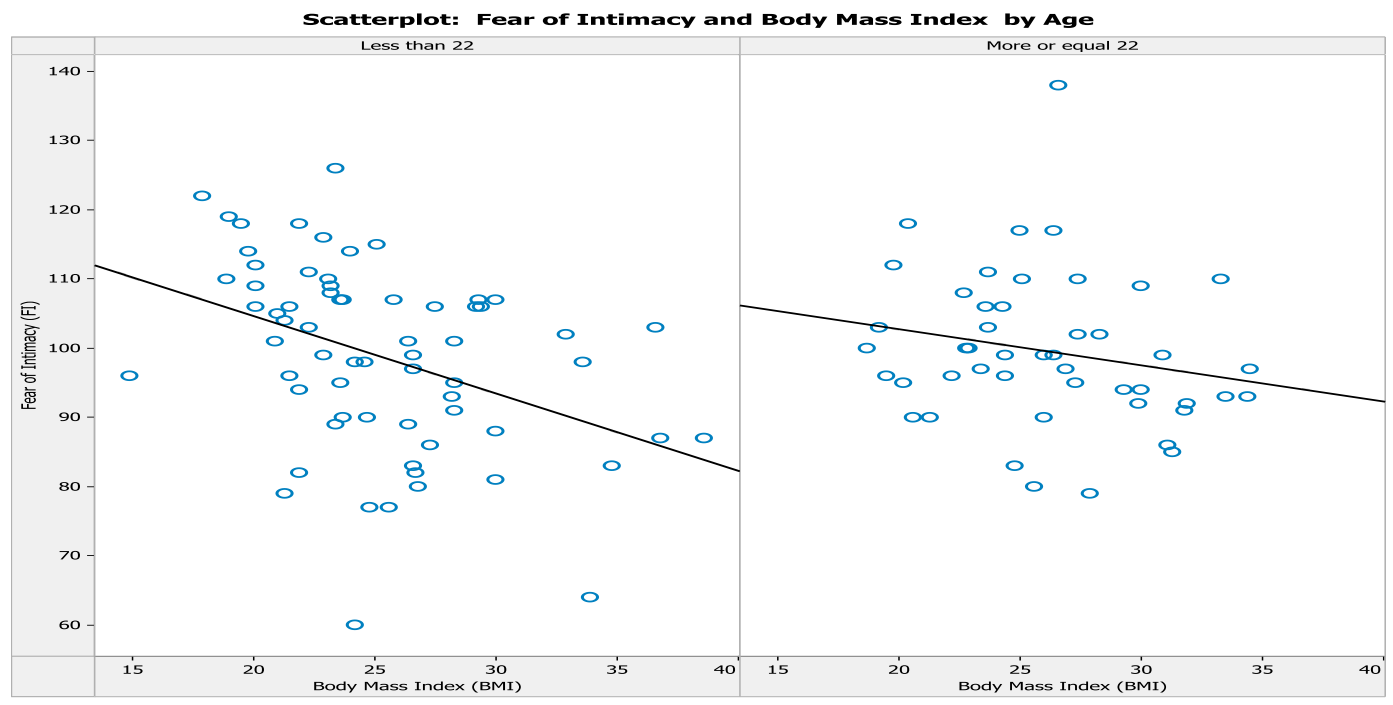

Fisher's R-to-Z transformation tested the difference between these correlations and yielded a $p$-value $=0.2853$, which does not provide evidence to reject the null hypothesis that the correlations between scores are equal for group 1 and group 2 . The sample sizes of $n 1=66$ and $n 2=47$ could also not be large enough for an effect size of $r=.30$. In this case, the null hypothesis that the correlations are not different is accepted (see Table 7). 
Hypothesis \#8: The relationship of body image avoidance and fear of intimacy varies from ages 18 to 22 (group 1) to ages 23 to 34 (group 2).

This hypothesis is supported; the correlation coefficient between FOI and BIA for Group1 sample is $-0.0148(p<0.9063)$. The correlation coefficient between FOI and BIA for Group 2 sample is $0.4139(p<0.0035)$ (see Table 6). This relationship is supported by the visual examination of the scatter plot between FOI and BIA for the population split by those who are less than 22 years old and those who are over 22 years of age (see Graph 7.2). The scatter plot of FOI and BIA for group 1 shows a weak negative relationship, while the scatter plot of FOI and BIA for group 2 reveals a strong positive relationship and could be a sign of major difference in the relationships between the two groups.

Graph 7.2.

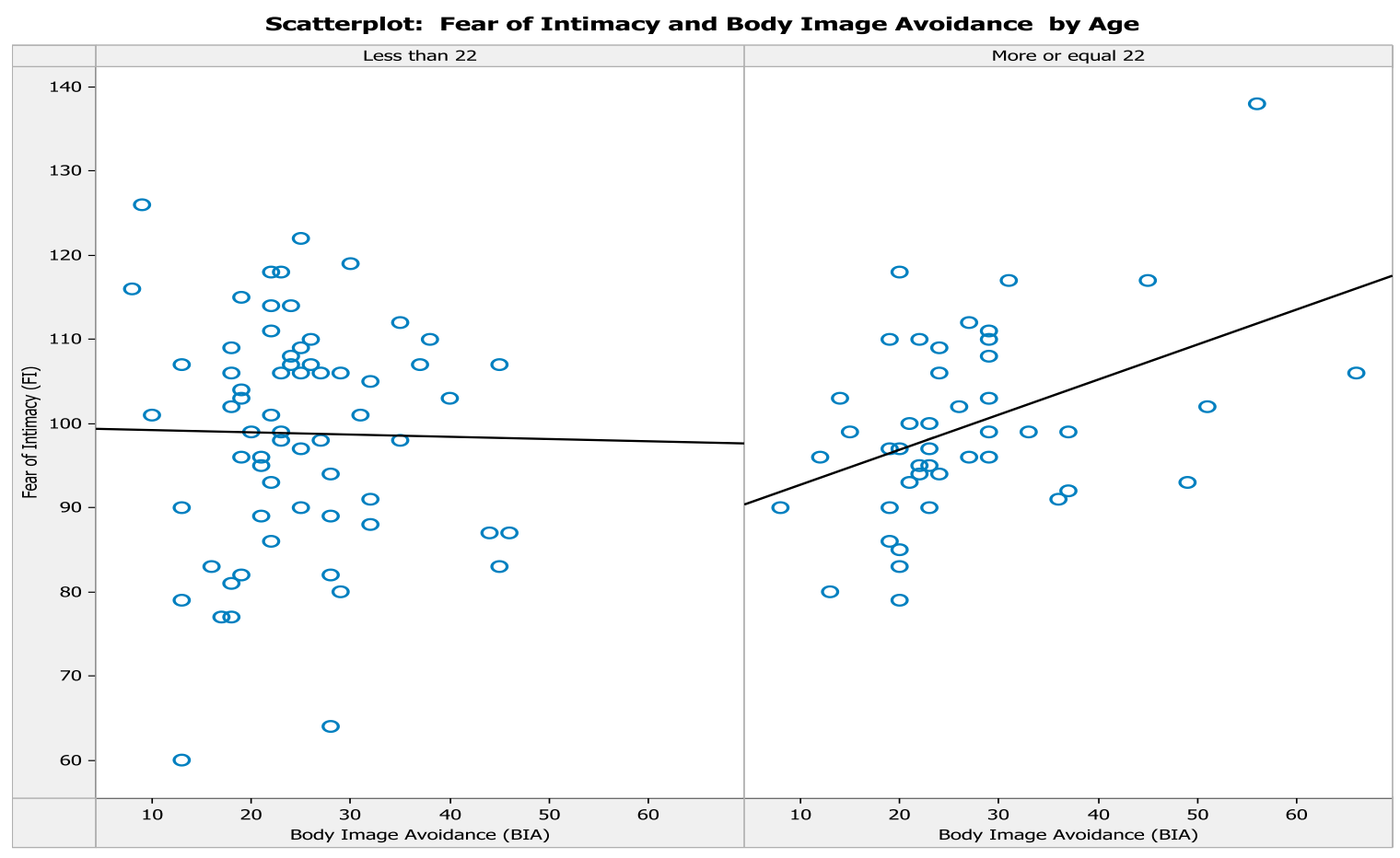


Fisher's R-to-Z transformation tested for the difference between these two correlations yielded a $p$-value $=0.0205$. This provides evidence to reject the null hypothesis that the correlations between scores are equal for group 1 and group 2 in favor of alternative hypothesis. In this case, the two correlations are different at a significance level of $r=.30$. (see Table 7).

Hypothesis \#9: The relationship of body mass index and body image avoidance varies from ages 18 to 22 (group 1) to ages 23 to 34 (group 2).

This hypothesis is not supported; the correlation coefficient between BIA and BMI for Group 1 is $0.3349(p<0.05)$. The correlation coefficient between BIA and BMI for Group 2 is $0.1845(\mathrm{p}<0.2157)$ (see Table 6). Visual examination of the correlation between BIA and BMI for group 1 and group 2 also does not show that these two correlations are significantly different (see Graph 7.3).

\section{Graph 7.3.}

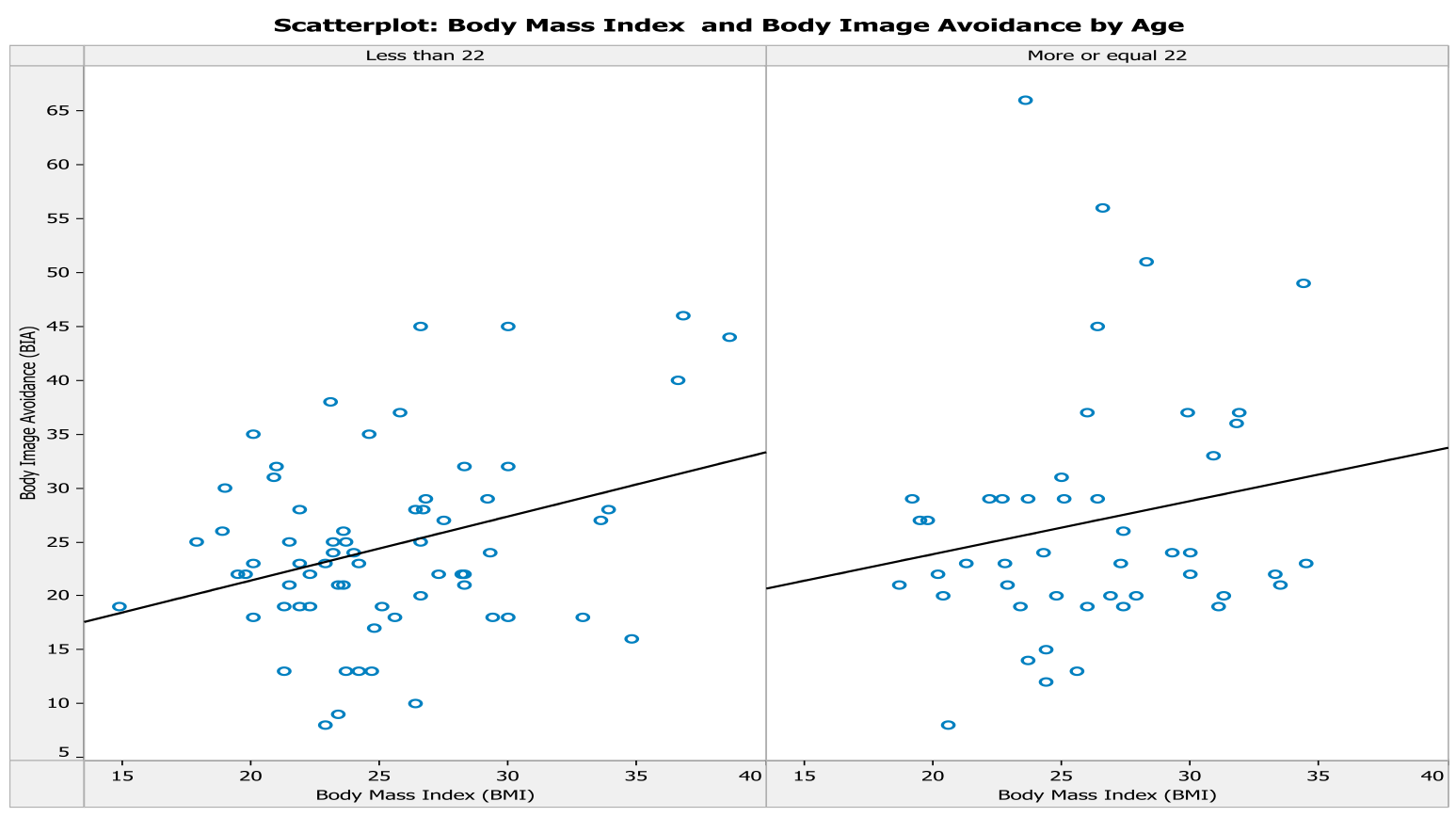


Fisher's R-to-Z transformation for the difference between these two correlations provided a $p$-value $=0.4104$ which does not offer evidence to reject the null hypothesis that the correlations between scores are equal for group 1 and group 2 (see Table 7). 


\section{CHAPTER V}

\section{DISCUSSION}

The primary purpose of this study sought to examine the relationship between a fear-of-intimacy and body image avoidance (attitudinal and perceptual), and how these relationships may be affected by body mass index. A secondary purpose sought to examine how these relationships may be gendered. Exploratory analysis of the collected data also indicated that age is involved in the relationships of body mass index, fear of intimacy and body image avoidance. Results indicated that as body mass index increases, fear of intimacy increases $(p<0.05)$ and that as body mass index increases, body image avoidance increases $(p<0.05)$. The relationship that as body image avoidance increases, fear of intimacy increases $(p<0.07)$ was also suggestive of a correlation, while not significant. No differences in these relationships were determined between gender groups. For age, the only observed relationship was that of a difference between scores for age groups [18 to 22 (group 1) and ages 23 to 34 (group 2)] for the relationship of body image avoidance and fear of intimacy $(p<0.02)$, with group 1 displaying increased body image avoidance and fear of intimacy.

The Relationship of Body Mass Index and Fear of Intimacy

The data revealed a correlation coefficient of -0.3261 (significant at $p<0.05$ ) for the relationship of body mass index and fear of intimacy. As a result, the hypothesis is accepted that as body mass index increases, fear of intimacy increases. The hypothesis that as body mass index increases, body image avoidance increases is also accepted as a 
result of the correlation coefficient $=0.2677$ (at $p<0.05)$. These findings are in line with research presented in the review of literature (Beller, 1977; Bess, 1997; Carpenter, et. al, 2000; Cash et al., 2004; Faith \& Schare, 1993; Schooler \& Ward, 2006; Strauss \& Wise, 1978) and speak to the close relationship of intimacy and body image.

The 1976 study cited by Beller hypothesized that adiposity served as a somatic metaphor for psychological armor that fat people employ as a defense against sex and love. The exploration of emotional issues that underlie obesity have been advocated by researchers toward effective treatment (Beach \& Martin, 1985). This may also explain, in part, the failure of community based interventions toward excessive weight since consumptive appetites are both subjective and personal in origin. The confirmation of these hypotheses offer motive to further investigate a reciprocal dynamism of these issues. The 2004 work by Cash et al. found that romantic attachment was the strongest predictor of body image dysfunction for men and women (Cash et al., 2004). The results of this aforementioned study confirmed significant relationships between interpersonal functioning and body image and would suggest that fear of intimacy (conceiving of intimacy as a romantic attachment) would predict body image avoidance (Cash et al., 2004). Body image disturbance has been found to characterize individuals with eating disorders, and has been found to exist with greater prevalence within overweight and obese individuals (Cash et al., 2004; Cash, 1993; Cash, 1990; Rodin et al., 1985; Garner, 1997).

Social constructs of masculinity and femininity hold both protective, and risk factors for the development of eating psychopathology, as well as guide attitudes and behaviors toward intimacy (Meyer, 2001). It has been speculated that perhaps this 
preoccupation is an attempt at upholding the virtues of power and control that sustain masculinity as a construct in a segment of the population often criticized as being effeminate (Heffernan, 1994). For homosexual men, visual cues are a powerful determinant for motivating the manifestations of body beautiful - the resulting effects yield an increased power in one's ability to realize romantic interaction. As constructs of masculinity continue to shift, and the new notion of the cosmopolitan "Metrosexual" becomes mainstream, the dynamics of gay relationships and attached impetus for achieving these physical ideals will offer greater insight into correlations between perceived body image and intimacy within our heterosexual society at large (Meyer, 2001).

Excessive weight (as defined by BMI) carries psychological consequences in developed societies that value thinness and physical attraction; the resulting affect may motivate avoidant cognitions and behaviors (Cash, 1993). Obese individuals overestimate or distort the size of their body more, are more dissatisfied and preoccupied with their appearance, and tend to avoid more social interactions because of their appearance than normal weight individuals (Cash, 1990). The accepted hypothesis in this study that as body mass index increases, fear of intimacy increases is supported by a correlation coefficient of -0.3261 (significant at $p<0.05$ ) and suggests that this relationship is linear, and that fear of intimacy correlated with BMI does not only occur at the point of obesity, but is relational.

As a result of many extraneous factors (including relationship status, relationship history, co-morbidities with psychological disorders, built environment, etc.) it is difficult to determine if fear of intimacy and body image avoidance result in an increased body 
mass index, or if the reverse is true. It would seem plausible that in a society that increasingly values thinness, the discrepancy between desirable body weight and actual body weight increases as well (Brownell, 2005). This would serve to intensify the bodyimage concerns of those who are objectively overweight and may result in a fear of intimacy. Changes in sexual interest and rate of sexual activity have been described coinciding with weight change (Strauss \& Wise, 1978) - however it is premature to attribute these changes to biology (Adolfsson et. al, 2004) or, instead, to socialized norms and expectancies.

The Relationship of Body Image Avoidance and Fear of Intimacy

The analysis of data further revealed a correlation coefficient of -0.1691 (at $p<0.07$ ), but did not lead to the acceptance of the hypothesis that as body image avoidance increases, fear of intimacy increases. Although the results indicate a weak positive correlation between BIA and FOI, the correlation was not significant at the $95 \%$ confidence level. The meta analytic study conducted by Cafri et al. (2005) determined that previous research into the influence of sociocultural factors on body image have commonly employed longitudinal and experimental designs to provide evidence for their role as causal risk factors for body image dissatisfaction. The study revealed that effect sizes obtained in cross-sectional studies are, on average, significantly larger than those obtained in experimental and longitudinal studies. This finding indicates that the magnitude of effects expected in experimental and prospective studies should be lower than those obtained in cross-sectional studies. These findings were carefully considered toward the planning of this study, but have also taken into account the complex issue of 
multiculturalism in the study of body image (Fitzgibbon et al., 2000). As a result of the strong relationship between these variables as addressed in the review of literature, and of Cafri et al.'s (2005) meta analysis, the weak positive correlation between body image avoidance and fear of intimacy is worthy of consideration.

Body image has been found to be closely related to the experience of romantic relationships and can affect both the quality and quantity of sexual and intimate experiences (Wiederman, 2002; Yamamiya, 2003, Cash et al., 2004). Internal (cognitive) models of adult attachment theories have long focused upon concepts of self-modeling and other-modeling (valuations), reflecting two basic dimensions of anxiety and avoidance of interaction (Cash et al., 2004). Avoidant behavior has also been examined as an external, behavioral component of body image for both men and women; selfperceptions of social attractiveness were positively related to the intimacy achieved in interaction (Nezlek, 1999). These consistent findings, including the confirmation of hypotheses in this study related to the relationships of fear of intimacy, body image avoidance and body mass index would suggest that these issues be examined to better understand the directionality of these relationships.

The rejection of the body has ramifications that extend far beyond the body. Research has indicated that for many women that feel "overweight," there is also an assumption of feeling unattractive, undesirable and ultimately - asexual (Areton, 2002). The resulting effect is one of withdrawal from trying to attract partners, from communicating or asserting themselves in both potential relationships and in actual sexual relationships (Areton, 2002). The suggestion has been made that weight bears 
psychosocial consequences that differ based upon gendered implications of weight (Carpenter, et. al, 2000).

As the social pressures for thinness and the associated stigmatization of obesity have increased, so has the rate of obesity (Friedman \& Brownell, 1995; "American Obesity Association", 2005; "Statistics," 2006). Among the overweight and obese segments of the population, as the pressure for thinness increases, the discrepancy between desirable body weight and actual body weight increases as well. This relationship has served to intensify the body-image concerns of those who are in fact overweight, and speculated that the greater the dissatisfaction, the greater the resulting avoidance toward social interaction (Friedman \& Brownell, 1995; “American Obesity Association”, 2005; Park, 2007). In spite of the growing body of research, little has been reported of the relationship between body image and body weight, nor how intimacy may be part of this equation. The results of this study indicate a weak positive correlation between BIA and FOI, and while not significant at the $95 \%$ confidence level, this correlation would be worthy of further investigation in future studies to better define any relationship between these two variables.

The Relationship of Gender and Fear of Intimacy, Body Mass Index, and Body Image Avoidance

Results did not indicate that the relationships of fear of intimacy, BMI and body image avoidance vary by gender. The $p$-values that ranged from 0.49 to 0.95 did not 
provide evidence to reject the null hypothesis that the correlations between scores are different for male and female. While prospective power analyses were conducted to estimate sample size and determined to require a minimum of 40 participants to achieve a medium desired effect size, the sample sizes $n_{1}=58$ (female) and $n_{2}=55$ (male) were not large enough to detect these differences at a significance level of 95\% percent. This is also evident from examining the scatter plots of scores by gender.

Little previous research has been conducted to examine the relationship of body image avoidance and fear of intimacy - less as it relates to body weight, and even less as it pertains to gender (Cash et al., 2004). This study found no significant difference between gender groups in the relationship of fear of intimacy and body mass index; this may be the result of too small a sample size to detect the desired effect size or of possible multicultural differences in the experience of body. While the study sample was derived from a multicultural campus environment, the confirmation of correlated results for both genders may reflect factors including acculturation, regional and generational differences as noted in the study by Fitzgibbon et al. (2000).

This lack of significance pertaining to gender would initially speak to an issue of sample size, the lack of significance may reveal important findings worthy of further investigation. The study's sample was obtained from Florida International University's multicultural campuses. According to the university's Office of Planning and Institutional Effectiveness, the fall 2007 university wide profile of students revealed the largest constituencies of 58.39\% Hispanic students, $13.18 \%$ African American students, and 17.65\% White students. The National Health and Nutrition Survey (NHANES) finds that 
the age-adjusted prevalence of overweight and obese combined in racial and ethnic minorities is generally higher than in Caucasians within the US ("Statistics," 2006); it is highest among non-Hispanic black women (77.3\% prevalence) and Hispanic American women (71.9 percent prevalence). This study sample held a mean BMI of 25.6. The 2000 Census found that the African American makeup of North Miami, a large community within north Miami-Dade consisted was $38.97 \%$. Despite a lack of available campusspecific data, Florida International University’s Biscayne Bay Campus is situated in this locale of North Miami and reflects this African American presence. The student body, and thus, a portion of the study sample, may reflect this community whose gendered differences may not reflect statistical significance as was hypothesized in this study.

The majority of research in the area of body image has not addressed the issue of body image (perceptual and attitudinal) in a stratified multi-ethnic population, nor has research established baselines for these findings (Fitzgibbon et al., 2000). Difficulties in body image research have been well documented (Cafri et al., 2005; Fitzgibbon et al, 2000, Rosen et al., 1991) as they pertain to race and ethnicity due to factors including acculturation, regional and generational differences, etc. A reflection of these findings may account for a lack of statistically significant gender differences in this study as the sample population does reflect a multiethnic university population as aforementioned.

Conversely, the lack of statistically significant variance between gender groups in this study may reflect important shifts in gender roles within a multiethnic population that deserve attention. The preoccupation of body image for men has often been obscured by the field's focus on thinness, which, although appropriate for the study of women, may 
inadequately capture men's body image preferences (Schooler \& Ward, 2006). Media and sociocultural markers now target men as an audience for the transmission of messaging pertaining to body image ideals (Faith \& Schare, 1993). This may also account for a lack of statistical difference between gender groups.

No study prior to Cash et al.'s 2004 work on body image and interpersonal functioning had examined body image and attachment in males. Similar to the results of this study, Cash et al. found little significant difference between gender groups. The results of the study (Cash et al., 2004) confirmed significant relationships between interpersonal functioning and body image - a reflection of growing social changes, gender role expectations, and a proliferation of media reflecting body ideals for both sexes (Cash et al., 2004).

The Relationship of Body Image Avoidance \& Fear of Intimacy Between Age Groups

Despite the initial treatment of only FOI, BIA, BMI and gender as variables, exploratory analyses revealed that differences in scores based upon age were also worthy of further investigation. Fisher's R-to- $Z$ transformation was used to test the difference between correlation coefficients between scores for two samples split by age. Since the sample population was comprised of students on a college campus, $95 \%$ of respondents were aged 28 or less. Prior to analysis, the additional variable of age was binned into two equal categories: group 1 (less than or equal to age 22) and group 2 (older than age 22). 
Of the correlations tested (BMI and BIA, FOI and BIA, FOI and BMI), the value for the difference of correlation between FOI and BIA that is equal to 0.02 provides evidence that these correlations for age group 1 and age group 2 are significantly different. Age has been found to be an important factor in classifying weight, height, and the resulting BMI from self-reports. An analysis of NHANES III data indicated statistically significant differences for the mean error (measured-self-reported values) for height and weight that were notably lower for younger $(<60)$ age groups (Kuczmarski et al., 2001). This is further support for the study sample to have consisted of participants aged 18 to 34 .

Research about body image highlights avenues toward generating meaningful insights. Cafri et al.'s (2005) meta-analysis suggested that greater attention be paid to an analysis of raw data. For example, age varies within each sample so that the mean age used as the moderator at the study level may lead to an underestimate of the actual moderating influence of age at the raw data level.

Cafri et al.'s (2005) meta analytic study revealed that neither age nor ethnicity was found to be a statistically significant moderator of the internalization-body image or awareness-body image relationship. Finding that age is not a significant moderator runs somewhat contrary to theories that adolescence is a critical period of development in which sociocultural influences are at their apex (Haines \& Neumark-Sztainer, 2006; Hargreaves \& Tiggemann, 2003; Harrison \& Cantor, 1997). For the purpose of this study, the relationship of FOI and BIA between age bins was significant only for those aged 22 or younger. It would be expected that as age increases, the association between 
sociocultural factors and body image would decrease (Haines \& Neumark-Sztainer, 2006; Hargreaves \& Tiggemann, 2003; Harrison \& Cantor, 1997). This expectation is evident in this study's examination of the relationship of fear of intimacy, body image avoidance and age and that a stronger relationship of FOI and BIA exists for those aged 18 to 22 .

Haines \& Neumark-Sztainer (2006) reported body dissatisfaction to be common among children and adolescents and considered how body image dissatisfaction and the avoidant behavior it spawns may be relevant in examining the development of obesity. The relationship of fear of intimacy and body image avoidance between age bins examined in this study demonstrates this trend (noting a stronger relationship between FOI and BIA for age bin 1). This may also speak to the inexperience with intimacy that is common in younger age cohorts, manifesting in avoidant behavior (and thus, a stronger relationship between FOI and BIA) as was suggested in the 2006 study by Haines \& Neumark-Sztainer.

It has been fairly well established, as well as demonstrated in this study, that weight as determined through self-reported BMI has less to do with body image than the attitudinal and perceptual body imagery of this weight (Areton, 2002; Friedman \& Brownell, 1995; Friedman et al., 2002). Instead, the relationships of fear of intimacy, body image avoidance and body mass index reveal correlations that may be useful toward more effective prevention and treatment in the North American epidemic of overweight and obesity by addressing issues that exist along with body weight. 


\section{Limitations}

The methodology employed in this study sought to reveal correlations between variables through quantifiable scales. The sample analyzed in this study revealed a correlational relationship between body mass index and intimacy, however, the nature of this relationship would benefit from a mixed method or qualitative approach to understanding the contextual qualities of these relationships. The research pertaining to body image and related spectrum of disorders have historically employed a quantitative approach, failing to consider the dynamism involved in these relationships. Qualitative data may reveal an improved understanding between interpersonal experiences and internal experiences.

Despite fluency in English amongst the university sample in this study, issues of intimacy, body image and body weight are highly subjective, and responses to the measures may have been biased by this, as well as by different interpretations of verbiage based on linguistic, cultural and religious differences. Intimacy, for example, means different things to different people, despite the treatment of specific behaviors and not attitudes presented in the validated measurement tools. Finally, while self-reported height and weight is the accepted practice in research pertaining to body image (Thompson, 2004) as body image is perceptual, this self reporting may account for some inaccuracies in the data. BMI may also fail to reveal true measurement of overweight and obesity based upon muscle mass, bone density and frame.

A further limitation worthy of appraisal in future research is the interaction between psychological issues and relational experiences. The treatment of these topics 
may better reveal antecedents and consequences of disordered body image, implications of body weight and relational experiences, rather than correlations alone. As a result of many extraneous factors (relationship status, relationship history, co-morbidities, etc.) it is difficult to determine if fear of intimacy and body image avoidance result in an increased body mass index, or if the reverse is true.

This study found no significant difference between gender groups in the relationship of fear of intimacy and body mass index - either as a result of sample size or of possible multicultural differences in the experience of body. Differences in these relationships between gender groups were hypothesized to reveal meaningful data, however, these differences were not detected. Larger samples of gender groups may have provided meaningful information based on greater sensitivity to smaller effect sizes. While the study sample was derived from a multicultural campus environment, the confirmation of correlated results for both genders may reflect factors including acculturation, regional and generational differences as noted in the study by Fitzgibbon et al. (2000). For research respective to body image, race and ethnicity, divergent ways of defining samples, inconsistent methods of measuring and categorizing weight status, and the use of differing body image measurements have contributed to problems in conclusive research, but also in the generalization of body image methodology and implications (Fitzgibbon et al., 2000).

The aim of this study was not to examine how race and ethnicity may impact attitudes toward body, but instead, how self-perception of body image may be correlated with fear of intimacy and body mass index, as well as to examine these gendered 
differences. Cafri et al. (2005)'s meta analytic study on body image found that ethnicity was not a statistically significant moderating effect for body image, however, it is clear that ethnicity may be a moderating effect for gender roles (Cafri et al., 2005; Fitzgibbon et al, 2000, Rosen et al., 1991). The student body, and thus, a portion of the study sample, may reflect this community whose gendered differences may not reflect statistical significance as was hypothesized in this study.

Limitations of this study exist, in part, as a result of current aforementioned limitations in methodology. In addition, this study's limitations exist in the lack of demographic information acquired, including additional information regarding relationship status, educational level, religious affiliations, ethnicity, economic status, etc.

\section{Future Research}

The explorations of emotional issues that underlie obesity have been advocated by researchers toward effective treatment (Beach \& Martin, 1985). This may also explain, in part, the failure of community based interventions toward excessive weight since consumptive appetites are both subjective and personal in origin. Results of this study indicated that as body mass index increases, fear of intimacy increases $(p<0.05)$ and that as body mass index increases, body image avoidance increases $(p<0.05)$. The confirmation of these hypotheses offers motive to further investigate a reciprocal dynamism of these issues, with a study design that can better support these correlations through larger sample sizes and reconsidered effect sizes. The relationship that as body image avoidance increases, fear of intimacy increases approached significance at $(p<0.07)$ but was not significant. 
Despite increasing research, little has been reported of the relationship between body image and body weight, nor how intimacy may be part of this equation. The results of this study indicate a weak positive correlation between BIA and FOI, and while not significant at the $95 \%$ confidence level, this potential relationship would be worthy of further investigation in future studies to better determine if a relationship exists. Contributions from psychological research over the past decade have failed to find inclusion of these issues in public health prevention and treatment approaches to obesity. Collaboration between the fields of expertise would provide a more comprehensive scope of the problem and opportunities for treatment and have been advocated by the Weight Control and Information Network of the National Institutes of Health ("Statistics", 2006).

Previous research has found that heavier women were less likely than their peers to get married (Gortmaker et al., 1993) and it was also determined that weight gain in women resulted in decreased sexual interest and satisfaction among their husbands while weight gain in men did not demonstrate a change in wives' sexual interest (Gortmaker et al., 1993; Margolin \& White, 1987). These changes have not been examined in homosexual relationships to the same extent. Studies have established a relationship between sexual orientation and eating psychopathology where homosexuality in men serves as a risk factor for the development of eating disorders, and serves as a protective factor in homosexual women (Heffernan, 1994; Meyer, 2001).

Social constructs of masculinity and femininity hold protective and risk factors for the development of eating psychopathology (Meyer, 2001) as well as body image dissatisfaction (Heffernan, 1994). For homosexual males, it has been speculated that perhaps this preoccupation is an attempt at upholding the virtues of power and control 
that sustain masculinity as a construct in a segment of the population often criticized as being effeminate (Heffernan, 1994). Accordingly, gender role and gender identity may provide meaningful data where gender alone failed to provide observed differences in this study. Between 1980 and 2000, the percentage of obese males increased from $13 \%$ to $28 \%$ and from $17 \%$ to $34 \%$ for women ("Statistics," 2006). This era reflects the aforementioned shifts in gender and sex roles in our modern history following the sexual revolution and women's liberation movement (Brumberg, 1988). The lack of statistical significance in differences between gender groups found in this study may reveal important changes in our society in which men and women are now both subject to body image related pressures, and share increasingly similar experiences that may be measured through smaller effect sizes, and thus, larger sample sizes.

Sexual orientation, gender identity (rather than assumptions based upon gender alone) and relationship status and relationship history may also provide insight as additional considerations in the discourse on the relationships of intimacy, body image and body mass index for future study.

\section{Implications}

The primary purpose of this study sought to examine the relationship between a fear-of-intimacy and body image avoidance (attitudinal and perceptual), and how these relationships may be affected by body mass index. Results indicated that as body mass index increases, fear of intimacy increases $(p<0.05)$ and that as body mass index increases, body image avoidance increases $(p<0.05)$. The relationship that as body image avoidance increases, fear of intimacy increases $(p<0.07)$ was also suggestive of a 
correlation, while not significant. A secondary purpose sought to examine how these relationships may be gendered; no differences in these relationships were found between gender groups in the study sample. These relationships have not been considered toward understanding what may be a cognitive reasoning for consumption that has contributed to the increase in rates of overweight and obesity since 1980 (Flegal, 2005; "Statistics," 2006). The results suggest that the relationship of body image avoidance and fear of intimacy, as well as age, bear consideration toward the escalating prevalence of overweight and obesity. An integrative approach to body weight that addresses issues of body image and intimacy may prove more effective in prevention and treatment.

The relationships addressed in this study provide a direction for further theoretical and applied work. While there are a number of variables that may come into play in the analysis of an increasing prevalence of overweight and obesity - including built environment, race, ethnicity, policy, culture, religion, media, access to opportunities for physical activity and proper nutrition, etc. - the individual experience of body image and its role in interpersonal functioning is an important consideration. Although a high degree of heritability for obesity exists, the rapid rise in the prevalence of overweight and obesity suggest that factors other than genetics may be involved (Chesney \& Thurston, 2001; Choi et al., 2005). The results of the study suggest that clinicians and behaviorists working with clients should consider the involvement of the relationship of body mass index, fear of intimacy and body image avoidance. The results from this study indicate that as body mass index increases, fear of intimacy increases and that as body mass index 
increases, body image avoidance increases. Therapies that address the interrelation of these issues may prove more effective than those that do not.

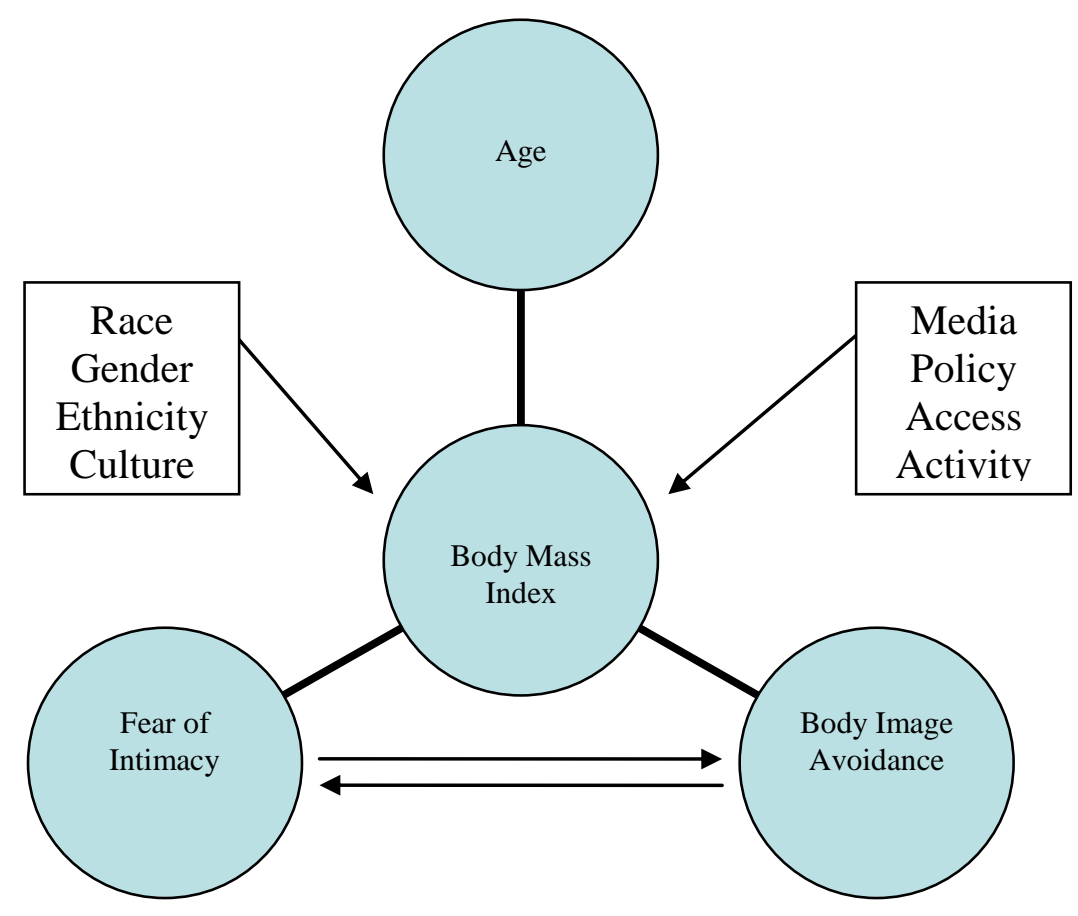

Most interventions to change health behaviors emphasize various strategies derived from theories of behavior as it occurs at the level of the individual. Nonetheless, individual behavior is continuously influenced by factors from the biological, psychological, social, and environmental levels (Lang, 2006). New paradigms are needed that examine the practice of health behaviors from initiation, through change, and into maintenance of change that recognize these influences. Similarly, to effectively change behavior and maintain change, efforts should focus on all of these levels. The most 
effective weight loss programs have empirically combined diet, exercise and behavioral modifications; however, even at best these programs have had only moderate success (Lang, 2006; Weaver \& Byers, 2006). While the psychoanalytic framework for this study is individualistic by nature, there are several implications for community-based health promotion and intervention efforts. The acknowledgement of a relationship between intimacy, body image and body mass index suggests a need to better understand how integrating therapies may benefit prevention and effective, long term weight loss strategies that ultimately affect that person's overall functioning

As overweight and obesity have become the norm ("Statistics", 2006), this described psychopathology concerning body image and intimacy may no longer be considered abnormal, but rather adaptive. This approach has begun to gain recognition as important for most patients who undergo rapid weight loss (gastric bypass surgery), because they must learn to accept and appreciate their bodies in order to enjoy close relationships with others (Areton, 2002). There are a number of healing therapies for the body (Hutchinson, 1985; Bergner, 1985; Tenzer, 1989; Freedman, 1988), but an integration of these therapies with nutritional counseling and physical activity have not been studied. Additional research in this area is necessary.

Body dissatisfaction among children and adolescents has been shown to result in avoidant behavior (Brumberg, 1988; Grogan, 1999; Haines \& Neumark-Sztainer, 2006). Avoidant behaviors, including isolation, often involve lower levels of physical activity and may bear implications later in life when forming intimate relationships (Haines \& Neumark-Sztainer, 2006). Body image dissatisfaction is well established as a risk factor 
for the development of eating disorders [according to the Diagnostic and Statistical Manual of Mental Disorders (DSM IV) criteria] but has rarely been considered toward the incidence of overweight and obesity. The relationships addressed in this study would suggest that for an adult population, body dissatisfaction is also correlated with avoidant behavior.

In looking to a 1997 study by Abraham and Llewellyn-Jones, 65\%-87\% of women between the ages of 20 and 60 were dissatisfied with their bodies due to a perception of too much fat, compared to $48 \%$ in 1972 . This statistic, which refers to a sample of women alone, provides an example that avoidant behavior concerning the body has increased along with average BMI ("Statistics", 2006). Treatment in the prevention of a dysphoric body image would include information from this study and review of literature in the field. The confirmation of the hypotheses that as BMI increases, fear of intimacy increases, and that as BMI increases, body image avoidance increases, offers motive to further investigate a reciprocal dynamism of these issues. If community based interventions toward excessive weight are the desired or feasible approach, these programs may obtain greater success if they are personalized and targeted toward specific communities rather than as national programs.

Research has moved toward considering body-image dissatisfaction as a powerful mediator for the emotional quality of life of obese individuals (Friedman \& Brownell, 1995; Jagsdaidt et al., 2001; Cash \& Pruzinsky 2002). The 2007 study by Park examined appearance-based rejection sensitivity as an extension of body image dissatisfaction and found that subjects who engaged in self-affirmation (thought of their personal strengths) 
or experienced a secure attachment through a close, caring relationship, were buffered from the negative effects of an appearance threat on subsequent self-esteem and mood. The study found that those preoccupied with their bodies would be motivated to engage in behaviors to appear attractive and avoid rejection by others and therefore were expected to be related to greater symptoms of disordered eating (Park, 2007). The results of this study, indicating a correlational relationship between fear of intimacy, body image avoidance and body mass index would suggest that these aforementioned behavioral selfaffirmations may also buffer the negative effects of body image avoidance and fear of intimacy which were found to be correlated with a higher body mass index.

Between 1980 and 2000, the percentage of obese males increased from $13 \%$ to $28 \%$ and from $17 \%$ to $34 \%$ for women ("Statistics," 2006). This era reflects the aforementioned shifts in gender and sex roles in our modern history following the sexual revolution and women's liberation movement (Brumberg, 1988). It is important to consider that the majority of the sample population, with a mean age of 21.6 , were born post-1980 and raised during this period of social change, flux in gender roles (with many mothers working away from the home), and during this period of rapid increase in the prevalence of an overweight population (Brumberg, 1988; Brumberg, 1997; Areton, 2002). The lack of statistical significance in differences between gender groups found in this study may reveal important changes in our society in which men and women are now both subject to body image related pressures, and share increasingly similar experiences. The lack of statistical difference between gender groups in this study highlights the importance that weight control is no longer a women's issue and that both gender groups 
be considered in researching, designing and implementing health promotion programs relevant to body image and body weight. Finally, processes of interpersonal interaction and communication relevant to intimacy and sensitive to gendered stereotypes must also be addressed so as not to ostracize men from such health programs.

Counseling and support groups focus on a sense of self-efficacy and internal validation as longer lasting rewards. Where public health serves as an intersection between such disciplines as psychology, public policy, sociology, anthropology, communications and marketing - a cohesive approach toward the eradication of overweight and obesity as a leading health issue must be adopted. In considering that obesity is a complex and multifaceted condition, new models for treatment and maintenance must consider underlying psychological cues along with comprehensive interventions. There are aspects of social validity and logic to be found in the psychoanalytic rationale for overweight and obesity that should not be overlooked. On a cultural level, in bringing to the attention the gendered rationale for body ideals, we may begin to become more aware of the psychological cues that gender roles implicate toward individual self-schema. Behaviorists and clinicians may draw from this study that avoidant behaviors may exacerbate issues in other areas, and that targeting these avoidant responses to issues of self image and ego may benefit the client. The results from this study indicate that as body mass index increases, fear of intimacy increases and that as body mass index increases, body image avoidance increases. While there are a number of therapies - size-acceptance therapies, shame-based therapies, self esteem and sexual therapies - to tackle the individual issues of intimacy and body image, as well as 
behavioral therapies to address body weight, none provide a holistic framework for the relationship of fear of intimacy, body image avoidance and body mass index (Areton, 2002). Therapies that address the interrelation of these issues may prove more successful toward achieving a healthy body mass index, and an improved attitude toward body image and intimacy. A focus on the individual will prove most effective, with an emphasis on internal validation and reward related to body image and a sense of intimacy with oneself (Areton, 2002). Cultural change may be too lengthy and ambitious a process in the face of this health epidemic. Focus on what may be achieved through personal development versus programmatic interventions that do not address such issues intimacy and body image have proven ineffective (Lang, 2006).

This study has shown that issues of body image and ability to achieve intimacy are relational to body weight, and that age is involved in these relationships. Communitybased programs may achieve better success in the prevention and intervention of overweight and obesity if highly targeted to specific audiences (Areton, 2002; Chesney \& Thurston, 2001; Haines \& Neumark-Sztainer, 2006; Tiggemann \& Lynch, 2001). The implications provide direction for further study, and for application in both individual and community-based approaches to address this national epidemic. 


\section{LIST OF REFERENCES}

Abraham, S., \& Llewellyn-Jones, D. (1997). Eating disorders: the facts (4th ed.). Oxford, England: Oxford University Press.

Adolfsson, B., et al. (2004). Are sexual dissatisfaction and sexual abuse associated with obesity? A population-based study. Obesity Research, 12:1702-1709.

American Obesity Association: New survey findings uncover the dramatic negative effects of severe obesity on intimacy, social interactions and employment opportunities (2005). Market Wire. Retrieved June 28, 2007 from http://findarticles.com/p/articles/mi_pwwi/is_200511/ai_n15750612 .

Areton, L. W. (2002). Factors in the Sexual Satisfaction of Obese Women Electronic Journal of Human Sexuality, 5: Jan. 15.

Argolin, L., \& White, L. (1987). The continuing role of physical attractiveness in marriage. Journal of Marriage and the Family, 49, 21-27.

Aubrey, J. (2007) The impact of sexually objectifying media exposure on negative body emotions and sexual self-perceptions: Investigating the mediating role of body self-consciousness, Mass Communication and Society, 10, 1, 1-23

Beach, V. \& Martin, D. (1985). Obesity, sexuality and psychological treatment for women. Women \& Therapy, 4, 1, 53-60.

Beller, A. S. (1978). Fat \& thin. New York: McGraw-Hill.

Bess, B.E. (1997). Human sexuality and obesity. International Journal of Mental Health, 26 (1), 61-67.

Bergner, M., Remer, P., \& Whetsell, C. (1985). Transforming women's body image: A feminist counseling approach. Women \& Therapy, 4, 3, 25-38.

Brennan, K.A., \& Shaver, P.R. (1995). Dimensions of adult attachment, affect regulation, and relationship functioning. Personality and Social Psychology Bulletin, 21, 267283.

Brown, P. (1993). Cultural perspectives on the etiology and treatment of obesity. In Stunkard, Albert \& Wadden, Thomas (Eds.), Obesity: Theory and therapy (2nd ed., pp. 179-191). New York, New York: Raven Press (Original work published 1993). 
Brownell, K.D. (2005). Does a toxic environment make obesity inevitable? Obesity Management, 1, 52-55.

Brumberg, J. J. (1988). Fasting girls. Harvard Univeristy Press: Cambridge, Massachusetts.

Brumberg, J. J. (1997). The body project. Random House: New York.

Cafri, G., Yamamiya, Y., Brannick, M., Thompson, K. (2005). The Influence of Sociocultural Factors on Body Image: A Meta-Analysis. Clinical Psychology: Science and Practice 12 (4), 421-433.

Carpenter, et al. (2000). Relationships between obesity and DSM-IV major depressive disorder, suicide ideation, and suicide attempts: results from a general population study. American Journal of Public Health, 90:251-57.

Cash, T. F., Maikkula, C. L., \& Yamamiya, Y. (2004). "Baring the body in the bedroom": Body image, sexual self-schemas, and sexual functioning among college women and men. Electronic Journal of Human Sexuality, 7. Retrieved October 5, 2006, from http://www.ejhs.org/volume7/bodyimage.html

Cash, T.F., Theriault, J., Annis, N.M. (2004). Body image in an interpersonal context: adult attachment, fear of intimacy, and social anxiety. Journal of Social \& Clinical Psychology; 23, 1, 89-103.

Cash, T. F. (1993). Body image attitudes among obese enrollees in a commercial weightloss program. Perceptual Motor Skills, 77, 1099-1103.

Cash, T. F. (1990). The psychology of physical appearance: aesthetics, attributes, and images. Body Images: Development, Deviance, and Change, 51-79, Guilford Press New York, NY.

Chen, Edwin H (1971). The Power of the Shapiro-Wilk W Test for Normality in Samples from Contaminated Normal Distributions. Journal of the American Statistical Association, Vol. 66, No. 336, pp. 760-762.

Chesney, M., \& Thurston, R. (2001). Creating social and public health environments to sustain behavior change: Lessons from obesity research. In N. Schneiderman, M.A. Speers, J.M. Silva, H. Tomes, \& J.H. Gentry (Eds.), Integrating behavioral and social sciences with public health (pp. 31-50). Washington, DC: American Psychological Association.

Choi, B.C.K., et al. (2005) Diseases of comfort: primary cause of death in the $21^{\mathrm{d}}$ century. Journal of Epidemiology and Community Health, 59, 12, 1030-1040. 
Cohane, G. H., \& Pope, H. G. (2001). Body image in boys: A review of the literature. International Journal of Eating Disorders, 29, 373-379.

Cohen, J. (1988). Statistical power analysis for the behavioral sciences (2nd ed.) Hillsdale, NJ: Lawrence Erlbaum Associates.

Crisp, A. H., \& McGuiness, B. J. (1976). Jolly fat: A relation between obesity and psychoneurosis in general population. Journal of British Medicine, 1, 7-9.

Descutner, C.J. \& Thelen, M. H. (1991) Development of a fear-of-intimacy scale. Psychological Assessment: A Journal of Consulting and Clinical Psychology, 3, 218-225.

Devlin, M.J., et al. (2000). Obesity: What mental health professionals need to know. American Journal of Psychiatry, 157, 854-866.

De Rios, M.D. (2001). Brief psychotherapy with the Latino immigrant client. New York: Haworth Press.

Drewnowski, A., \& Yee, D. K. (1987). Men and body image: Are males satisfied with their body weight? Psychosomatic Medicine, 49, 626-634.

Erdfelder, E., Faul, F., \& Buchner, A. (1996). GPOWER: A general power analysis program. Behavior Research Methods, Instruments, \& Computers, 28, 1-11.

Faith, M. S., \& Schare, M. L. (1993). The role of body image in sexually avoidant behavior. Archives of Sexual Behavior, 22, 4, 345-356.

Falicov, C.J. (1998). Latino families in therapy. New York: Guilford Press.

Fishbein, M., \& Ajzen, I. (1975). Belief, attitude, intention behavior: An introduction to theory and research. Reading, MA: Addison-Wesley.

Fitzgibbon, M.L., Blackman, L. \& Avellone, M. (2000). The relationship between body image discrepancy and body mass index across ethnic groups. Obesity Research, $8,582-589$.

Flegal, K. (2005) Epidemiologic aspects of overweight and obesity in the United States. Physiology \& Behavior, 86, 5, 599-602.

Ford, C. S., \& Beach, F. A. (1980). Patterns of sexual behavior. Westport, Ct: Greenwood Press.

Freedman, R. (1989). Bodylove. New York: Harper \& Row. 
Friedman, M.A., \& Brownell, K.D. (1995) Psychological correlates of obesity: moving to the next research generation. Psychology Bulletin, 117, 3-20.

Friedman, K.E., Reichmann, S., Costanzo, P., Musante, G. (2002). Body image partially mediates the relationship between obesity and psychological distress. Obesity Research, 10, 33-41.

Freud, S. (1962). Three Essays on the Theoryof Sexuality, trans. James Strachey. New York: Basic Books.

Garner, D. (1991). Eating Disorder Inventory-2. Odessa, FL: Psychological Assessment Resources.

Garner, D.M. (1997). The body image survey results. Psychology Today 30, 75-84.

Gortmaker, S.L., Must, A., Perrin, J.M., Sobol, A.M., \& Dietz, W.H. (1993) Social and economic concequences of overweight in adolescence and young adulthood. New England Journal of Eating Disorders, 18, 91-97.

Greenfield, S., \& Thelen, M. (1997). Validation of the fear of intimacy scale with a lesbian and gay male population. Journal of Social and Personal Relationships, 14, 5, 707-716.

Grogan, S. (1999). Body image: Understanding body dissatisfaction in men, women, and children. New York: Routledge.

Ginis, K. A. M., et al. (2005). Mind over muscle? Sex differences in the relationship between body image change and subjective and objective physical changes following a 12-week strength-training program. Body Image, 2, 362-272.

Hargreaves, D., \& Tiggemann, M. (2002). The role of appearance schematicity in the development of adolescent body dissatisfaction. Cognitive Therapy \& Research, 26, 691-700.

Harrison, K., \& Cantor, J. (1997). The relationship between media consumption and eating disorders. Journal of Communication, 47, 40-67.

Hart, T.A., et al. (2008). Development and examination of the social appearance anxiety scale. Assessment, 15; 48-59.

Hebl, M. R. et al. (2004). The swimsuit becomes us all: Ethnicity, gender, and vulnerability to self-objectification. Personality and Social Psychology Bulletin, $30,1322-1331$. 
Heffernan, K. (1994). Sexual orientation as a factor in risk for binge eating and bulimia nervosa: A review. International Journal of Eating Disorders, 16, 335-347.

Hein, D., Haas, G., \& Cook, H. (1998).Gender differences in premorbid social adjustment and intimacy motivation in schizophrenia. Journal of Clinical Psychology, 54, 1, 35-48.

Hochbaum, G. M. (1958). Public participation in medical screening programs: A sociopsychological study. Public Health Service Publication no. 572. Washington, DC.

Holland, J., Ramazanoglu, C., Scott, S., Sharpe, S., \& Thompson, R. (1990). Sex, gender, and power: Young women's sexuality in the shadow of AIDS. Sociology of Health \& Illness, 3, 336-350.

Hutchinson, M. G. (1985). Transforming body image. Freedom, California: The Crossing Press.

Jagsdaidt et al. (1997) Relationships between sexuality and obesity in male patients. New Trends in Experimental and Clinical Psychiatry, 13, 2, 105-110.

Jagsdaidt et al. (2001). Relationships between sexuality and obesity in female patients. New Trends in Experimental and Clinical Psychiatry, 17, 1, 69-77.

Kenrick, D. T., \& Gutierres, S. E. (1980). Contrast effects and judgments of physical attractiveness: When beauty becomes a social problem. Journal of Personality and Social Psychology, 38, 131-140.

Kolotkin, R.L., et al. (2006). Obesity and sexual quality of life. Obesity 14, 472-479.

Kumanyika S., Wilson JF, Guilford-Davenport M (1993). Weight-related attitudes and behaviors of black women. Journal of the American Dietetic Association. Apr 93, 4, 416-22.

Lang, A., \& Froelicher, E.S. (2006). Management of overweight and obesity in adults: Behavioral intervention for long-term weight loss and maintenance. European Journal of Cardiovascular Nursing, 5,102-114.

Levine, M. J. (1997). I Wish I Were Thin...I Wish I Were Fat: The Real Reasons We Overeat \& What We Can Do About It. Vanderbilt Press: New York.

Lipsey, M.L. (1990). Design Sensitivity: Statistical Power for Experimental Research. Sage Publications, Newbury Park, CA. 
Lips, H. M. (2001). Sex and gender: An introduction (4th Ed.). Toronto: Mayfield Publishing.

Lovejoy, M. (2001) Disturbances in the Social Body: Differences in body image and eating, problems among African American and White women. Gender and Society, 15, 2, 239-261.

Meyer, C. (2001). Sexual Orientation and eating psychopathology; the role of masculinity and femininity. The International Journal of Eating Disorders, 29, 3, 314-318.

Miller, C.H. (1970). Fat and fed up; the challenge of weight control. New York: Lyle Stuart.

Miller, R. S., \& Lefcourt, H. M. (1982). The assessment of social intimacy. Journal of Personality Assessment, 46(5), 514-518.

Miller, S (1979). Food for thought, a new look at food and behavior. Englewood Cliffs, NJ: Prentice Hall.

Mokdad, A.H., et al. (2000). The continuing epidemic of obesity in the United States. Journal of the American Medical Association, 284, 1650-1651.

Mussap, A. (2007). Short Communication: The relationship between feminine gender role, stress and disordered eating symptomatology in women. Stress and Health 23: $343-348$.

Newton, M., et al. (2005). Romantic relationships for women with anorexia nervosa: an integrative literature review. Eating and Weight Disorders, 103, 139-153.

Nezlek, J.B. (1999). Body image and day-to-day social interaction. Journal of Personality, 67, 793-816.

Park, L.E. (2007). Appearance-based rejection sensitivity: Implications for mental and physical health, affect, and motivation. Personality and Social Psychology Bulletin, 33, 490-510.

Parker, S., M. Nichter, C.S. Vuckovic, et al. (1995). Body image and weight concerns among African-American and white adolescent females: Differences that make a difference. Human Organization, 54, 103-14.

Prochaska, J. O., \& DiClemente, C. C. (1983). Stages and processes of self-change of smoking: Toward an integrative model of change. Journal of Consulting and Clinical Psychology, 51, 390-395. 
Psychoanalysis (2006). Wikipedia. Retrieved October 24, 2006 from http://en.wikipedia.org/wiki/Psychoanalysis\#Cultural_Adaptations.

Roberts, W.C. (2004). The Amish, body weight and exercise. The American Journal of Cardiology, 94, 9, 1221.

Rodin, J., Silberstein, L., Striegel-Moore, R. (1985) Women and weight: a normative discontent Psychology and Gender: Nebraska Symposium on Motivation 32, 267307.

Rosen, J.C., Srebnik, D., Saltzberg, E., \& Wendt, S. (1991) Development of a body image avoidance questionnaire. Psychological Assessment: A Journal of Consulting and Clinical Psychology, 3, 32-37.

Rosenstock, I. M. (1960). What research in motivation suggests for public health. American Journalof Public Health, 50, 295-301.

Rosenstock, 1. M. (1966). Why people use health services. Milbank Memorial Fund Quarterly, 44, 94-124.

Rosenstock, I. M. (1974). Historical origins of the Health Belief Model. Health Education Monographs, 2, 328-335.

Schembri, C., \& Evans, L. (2008). Adverse relationship processes: The attempts of women with bulimia nervosa symptoms to fit the perceived ideal of intimate partners. European Eating Disorders Review, 16, 59-66.

Schooler, D. \& Ward, L. M. (2006). Average Joes: Men's relationships with media, real bodies, and sexuality. Psychology of Men \& Masculinity, 7, 1, 27-41.

Shapiro, S.L. (1980). Sexual attitudes and activities of obese women. Unpublished doctoral dissertation, The Institute for the Advanced Study of Human Sexuality.

Silberstein, L. R., Striegel-Moore, R. H., Timko, C., \& Rodin, J. (1988). Behavioral and psychological implications of body dissatisfaction: Do men and women differ? Sex Roles, 19, 219-232.

Smuts, R.W. (1992). Fat, sex, class, adaptive flexibility and cultural change. Etiology and Sociobiology, 13, 5, 523-542.

Spiegel, J. (1988). Differences between obese women in a relationship and obese women not in a relationship. Unpublished doctoral dissertation, California Graduate School of Marital and Family Therapy. 
Statistics: overweight and obesity (2006). Weight control and information network. Retrieved March 15, 2006 from http://win.niddk.nih.gov/statistics/index.htm

Stern, Steven (2001). Sexuality and mass media: the historical context of psychology's reaction to sexuality on the internet. Journal of Sex Research, November 2001. Retrieved March 1, 2007 from http://www.findarticles.com/p/articles/mi_m2372/is_4_38/ai_84866946.

Strauss R.J., \& L. Wise (1978). Operative risks of obesity. Surgery, Gynecology \& Obstetrics, 146, 286-91.

Swift, S.E. (2005). The effects of thin-ideal images and feminine gender role stress on body satisfaction, mood, and cognitions in women. Dissertation Abstracts International: Section B: The Sciences and Engineering, 65(8-B). US: Pro-Quest Information \& Learning.

Thompson, J.K. (1991). Body shape preferences: Effects of instructional protocol and level of eating disturbance. International Journal of Eating Disorders, 10, 193198.

Thompson, J.K., et al., (1999). Exacting beauty: Theory, assessment, and treatment of body image disturbance. Washington, DC: American Psychological Association.

Thomspon, J.K. (2004). The (mis)measurement of body image: Ten strategies to improve assessment for applied and research purposes. Body Image, 1,1, 7-14

Tiggemann, M., \& Lynch, J. E. (2001). Body image across the life span in adult women: The role of self-objectification. Developmental Psychology, 37, 243-253.

Tiggerman, M. (2005). Body dissatisfaction, disordered eating, fashion magazines and clothes; a cross-cultural comparison between Australian and Italian young women. International Journal of Psychology, 40, 5, 293-302.

Vander Wal, J.S. (2004). Eating and body image concerns among average-weight and obese African American and Hispanic Girls. Eating Behaviors; An International Journal, 5, 2, 181-187.

Vander Wal, J.S., \& Thomas, N. (2004). Predictors of body image dissatisfaction and disturbed eating attitudes and behaviors in African American and Hispanic girls. Eating Behaviors; An International Journal, 5, 4, 291-301.

Vega, W.A. (1990). Hispanic families in the 1980s: A decade of research. Journal of Marriage and the Family, 52, 1015 - 1024. 
Wadden, T.A., \& Phelan, S. (2001). Assessment of quality of life in obese individuals. Obesity Research, 10, 1, 50-57.

Weaver, A., \& Byers, E.S. (2006). The relationships among body image, body mass index, exercise, and sexual functioning. Psychology of Women Quarterly, 30, 333-339.

Wiederman, M.W. (2002). Body image and sexual functioning. In T.F. Cash \& T Pruzinsky (Eds.), Body image: A handbook of theory, research, and clinical practice (pp. 287-294). New York: Guildford Press.

Wiederman, M.W., \& Hurst, S.R. (1997). Physical Attractiveness, Body Image, and Women's Sexual Self-Schema. Psychology of Women Quarterly, 21, 567-580.

Weinfeld, M., Sigal, J.J., \& Eaton, W.W. (1981) Long-Term Effects of the Holocaust on Selected Social Attitudes and Behaviors of Survivors: A Cautionary Note. International Journal of Social Research, 60, 1, 1-19.

Wilcox, C. (1991) Race, gender role attitudes and support for feminism. The Western Political Quarterly, 43, 1, 113-121.

Wise, T. N. (1978). Massive obesity \& sexual activity. Medical Aspects of Human Sexuality, 7-23. 


\section{APPENDICES}

Measure: $\quad$ Fear-of-Intimacy Scale (FIS; Descutner \& Thelen, 1991)

Part A Instructions: Imagine you are in a close, dating relationship. Respond to the following statements as you would if you were in that close relationship. Rate how characteristic each statement is of you on a scale of 1 to 5 as described below, and put your response in the space to the left of the statement.

$$
\begin{aligned}
& 1=\text { Not at all characteristic of me } \\
& 2=\text { Slightly characteristic of me } \\
& 3=\text { Moderately characteristic of me } \\
& 4=\text { Very characteristic of me } \\
& 5=\text { Extremely characteristic of me }
\end{aligned}
$$

Note. In each statement " $\mathrm{O}$ " refers to the person who would be in the close relationship with you.

1. I would feel uncomfortable telling $\mathrm{O}$ about things in the past that I have felt ashamed of.

2. I would feel uneasy talking with $\mathrm{O}$ about something that has hurt me deeply.

3. I would feel comfortable expressing my true feelings to $\mathrm{O}$.

4. If $\mathrm{O}$ were upset I would sometimes be afraid of showing that I care.

5. I might be afraid to confide my innermost feelings to $O$.

6. I would feel at ease telling $\mathrm{O}$ that I care about him/her.

7. I would have a feeling of complete togetherness with $\mathrm{O}$.

8. I would be comfortable discussing significant problems with $O$.

9. A part of me would be afraid to make a long-term commitment to $\mathrm{O}$.

10. I would feel comfortable telling my experiences, even sad ones, to $\mathrm{O}$.

11. I would probably feel nervous showing $\mathrm{O}$ strong feelings of affection.

12. I would find it difficult being open with $\mathrm{O}$ about my personal thoughts.

13. I would feel uneasy with $O$ depending on me for emotional support.

14. I would not be afraid to share with $\mathrm{O}$ what I dislike about myself.

15. I would be afraid to take the risk of being hurt in order to establish a closer relationship with $\mathrm{O}$.

16. I would feel comfortable keeping very personal information to myself.

17. I would not be nervous about being spontaneous with $O$.

18. I would feel comfortable telling $O$ things that I do not tell other people.

19. I would feel comfortable trusting $O$ with my deepest thoughts and feelings.

20. I would sometimes feel uneasy if $O$ told me about very personal matters.

21. I would be comfortable revealing to $\mathrm{O}$ what I feel are my shortcomings and handicaps.

22. I would be comfortable with having a close emotional tie between us.

23. I would be afraid of sharing my private thoughts with $O$.

24 . I would be afraid that I might not always feel close to $O$. 
25 . I would be comfortable telling $\mathrm{O}$ what my needs are.

26. I would be afraid that $O$ would be more invested in the relationship than I would be.

27. I would feel uncomfortable about having open and honest communication with $\mathrm{O}$.

28. I would sometimes feel uncomfortable listening to O's personal problems.

29. I would feel at ease to completely be myself around $O$.

30. I would feel relaxed being together and talking about our personal goals.

Part B Instructions: Respond to the following statements as they apply to your past relationships. Rate how characteristic each statement is of you on a scale of 1 to 5 as described in the instructions for Part A.

31. I have shied away from opportunities to be close to someone.

32. I have held back my feelings in previous relationships.

33. There are people who think that I am afraid to get close to them.

34. There are people who think that I am not an easy person to get to know.

35. I have done things in previous relationships to keep me from developing closeness.

Source: Corcoran, K., \& Fischer, J. (2000). Measures for Clinical Practice: A Sourcebook (Vol. 2). New York, NY: The Free Press. 
Body Image Avoidance Questionnaire

Rosen, J.C., Srebnik, D., Saltzberg, E., \& Wendt, S. (1991) Development of a body image avoidance questionnaire. Psychological Assessment: A Journal of Consulting and Clinical Psychology, 3, 32-37.

\section{Appendix}

\section{Body Image Avoidance Questionnaire}

Circle the number which best describes how often you engage in these behaviors at the present time.

\begin{tabular}{|c|c|c|c|c|c|c|}
\hline & Always & Usually & Often & Sometimes & Rarely & Never \\
\hline I. I wear baggy clothes & 5 & 4 & 3 & 2 & 1 & 0 \\
\hline 2. I wear clothes I do not like & 5 & 4 & 3 & 2 & 1 & 0 \\
\hline 3. I wear darker color clothing & 5 & 4 & 3 & 2 & 1 & 0 \\
\hline \multicolumn{7}{|l|}{ 4. I wear a special set of clothing, e.g., my "fat } \\
\hline clothes" & 5 & 4 & 3 & 2 & 1 & 0 \\
\hline 5. I restrict the amount of food I eat & 5 & 4 & 3 & 2 & 1 & 0 \\
\hline \multicolumn{7}{|l|}{ 6. I only eat fruits, vegetables and other low calorie } \\
\hline foods & 5 & 4 & 3 & 2 & 1 & 0 \\
\hline 7. I fast for a day or longer & 5 & 4 & 3 & 2 & i & 0 \\
\hline 8. I do not go out socially if I will be "checked out" & 5 & 4 & 3 & 2 & 1 & 0 \\
\hline \multicolumn{7}{|l|}{ 9. I do not go out socially if the people I am with } \\
\hline will discuss weight & 5 & 4 & 3 & 2 & 1 & 0 \\
\hline \multicolumn{7}{|l|}{ 10. I do not go out socially if the people I am with } \\
\hline are thinner than me & 5 & 4 & 3 & 2 & 1 & 0 \\
\hline I1. I do not go out socially if it involves eating & 5 & 4 & 3 & 2 & $i$ & 0 \\
\hline 12. I weigh myself & 5 & 4 & 3 & 2 & $i$ & 0 \\
\hline 13. I am inactive & 5 & 4 & 3 & 2 & 1 & 0 \\
\hline 14. I look at myself in the mirror & 5 & 4 & 3 & 2 & 1 & 0 \\
\hline 15. I avoid physical intimacy & 5 & 4 & 3 & 2 & 1 & 0 \\
\hline \multicolumn{7}{|l|}{ 16. I wear clothes that will divert attention from my } \\
\hline weight & 5 & 4 & 3 & 2 & 1 & 0 \\
\hline 17. I avoid going clothes shopping & 5 & 4 & 3 & 2 & 1 & 0 \\
\hline \multicolumn{7}{|l|}{ 18. I don't wear "revealing" clothes (e.g., } \\
\hline bathingsuits, tank tops, or shorts) & 5 & 4 & 3 & 2 & 1 & 0 \\
\hline 19. I get dressed up or made up & 5 & 4 & 3 & 2 & 1 & 0 \\
\hline
\end{tabular}


Demographic Information:

Age:

Gender:

Please indicate, to the best of your ability:

Height

Weight 
VITA

\section{ELLIOT MONTGOMERY SKLAR}

2008:

Pre-Health Advisor

Pre-Health Professions Advising Center, University Park

Florida International University, Miami, FL

2004-2007: $\quad$ Career Consultant

Office of Career Services, Biscayne Bay Campus

Florida International University, Miami, FL

2004: $\quad$ Master of Science in Mass Communication

Florida International University, Miami, FL

2004: Research Assistant

Gastroenterology Unit

Montreal Jewish General Hospital, Montreal, PQ

2003: $\quad$ Independent Student

Concordia University, Montreal, PQ

2002: $\quad$ Bachelor of Arts in Psychology

Concordia University, Montreal, PQ

1999: $\quad$ Diploma of Collegial Studies (DEC) in Creative Arts

Dawson College, Montreal, PQ

\section{SELECTED PROFESSIONAL PRESENTATIONS AND PUBLICATIONS}

Sklar, E. (2007, September). The relationship of fear of intimacy, body image avoidance and body mass index: A review of literature. Presentation at $3^{\text {rd }}$ Annual Symposium on Addictive \& Health Behaviors. Amelia Island, FL.

Farber, L. \& Sklar, E. (2006, March). The effects of direct-to-consumer advertising for pharmaceuticals. Presentation at $9^{\text {th }}$ Annual Public Relations Conference, Salt Lake City, UT. 


\section{HONORS AND AWARDS}

- Florida International University Wide Scholarship (2006-2007)

- Florida International University Student Government Association Award Recipient (2007)

- Florida - Canada Linkage Scholar (2004 -2006)

- Aventura Marketing Council Scholarship Award (2005) 